Supporting Information for

\title{
N-Methylation of Amines with Methanol in Aqueous Solution Catalyzed by a Water-Soluble Metal-Ligand Bifunctional Dinuclear Iridium Catalyst
}

\author{
Chong Meng, ${ }^{\dagger}$ Peng Liu, ${ }^{\dagger}$ Nguyen Thanh Tung, ${ }^{\dagger}$ Xingyou Han,${ }^{\dagger}$ and Feng Li ${ }^{*}, \dagger^{\dagger}$ \\ †School of Chemical Engineering, Nanjing University of Science \& Technology, Nanjing 210094, \\ P. R. China \\ ${ }^{\ddagger}$ State Key Laboratory of Fine Chemicals, Dalian University of Technology, Dalian 116024, P. R. China \\ *E-mail: fengli@njust.edu.cn
}

Table of Contents:

Copies of ${ }^{1} \mathrm{H}$ NMR and ${ }^{13} \mathrm{C}$ NMR spectra of products S2-S69

NMR data for the labelling experiments

S70-71 
$\mathrm{N}$-methylbenzenesulfonamide

Proton CDCl3
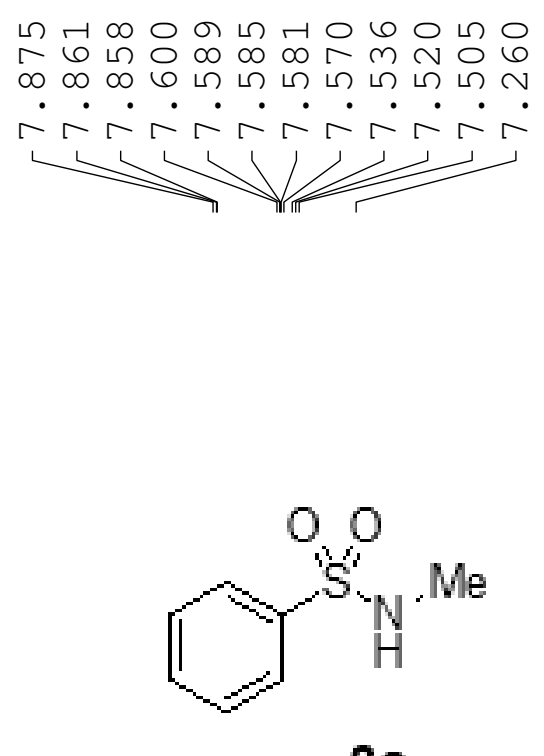

$2 a$

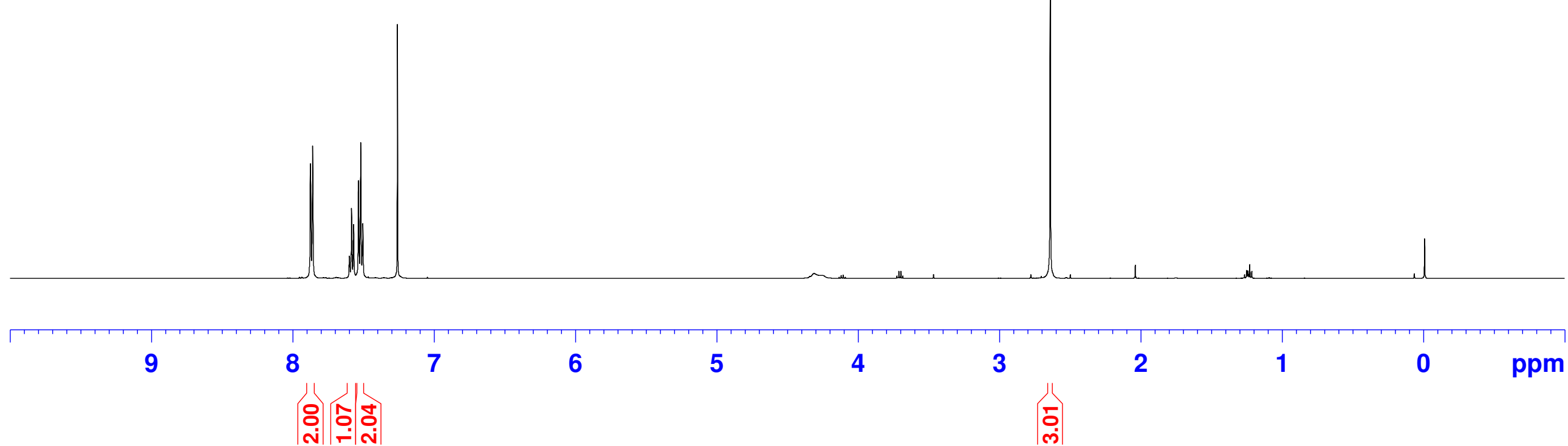


$\mathrm{N}$-methylbenzenesulfonamide C13CPD CDCl3
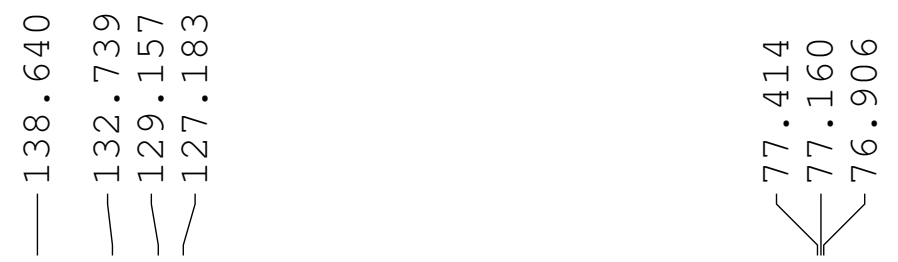

<smiles>CNS(=O)(=O)c1ccccc1</smiles>

$2 a$

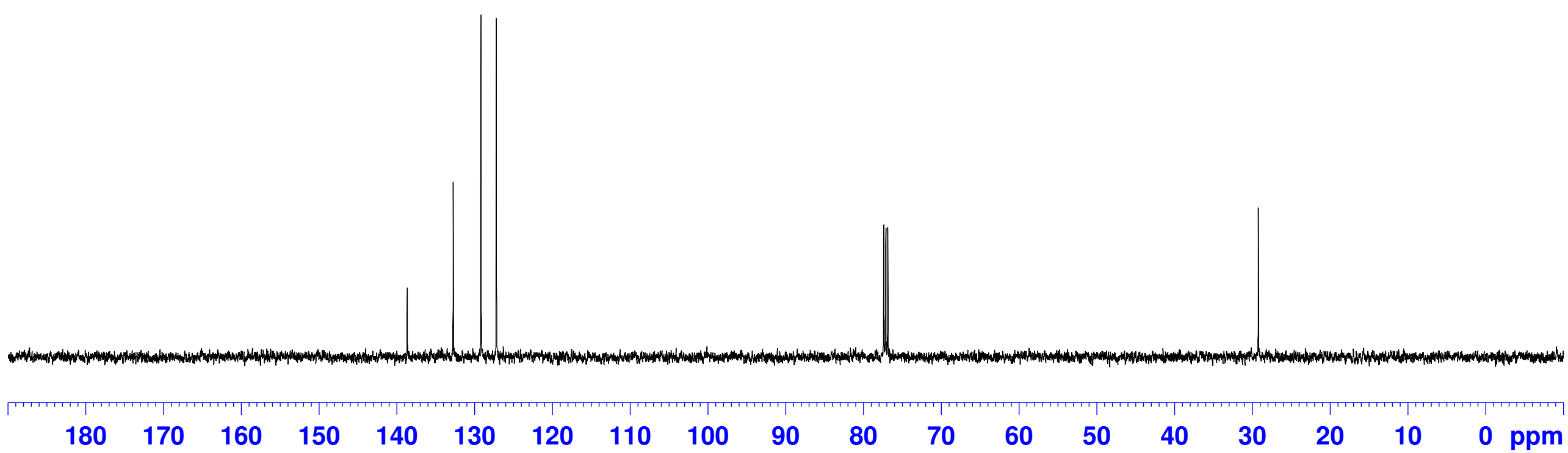


2-fluoro-N-methylbenzenesulfonamide Proton $\mathrm{CDCl} 3$

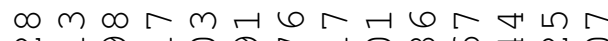

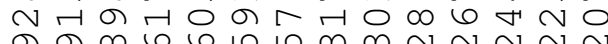

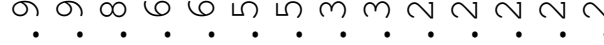

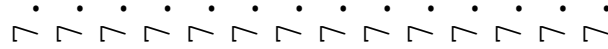
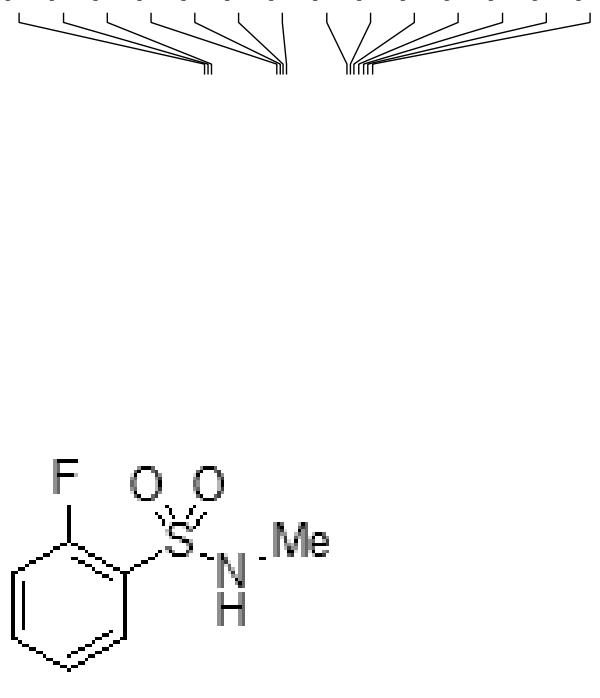

$2 b$
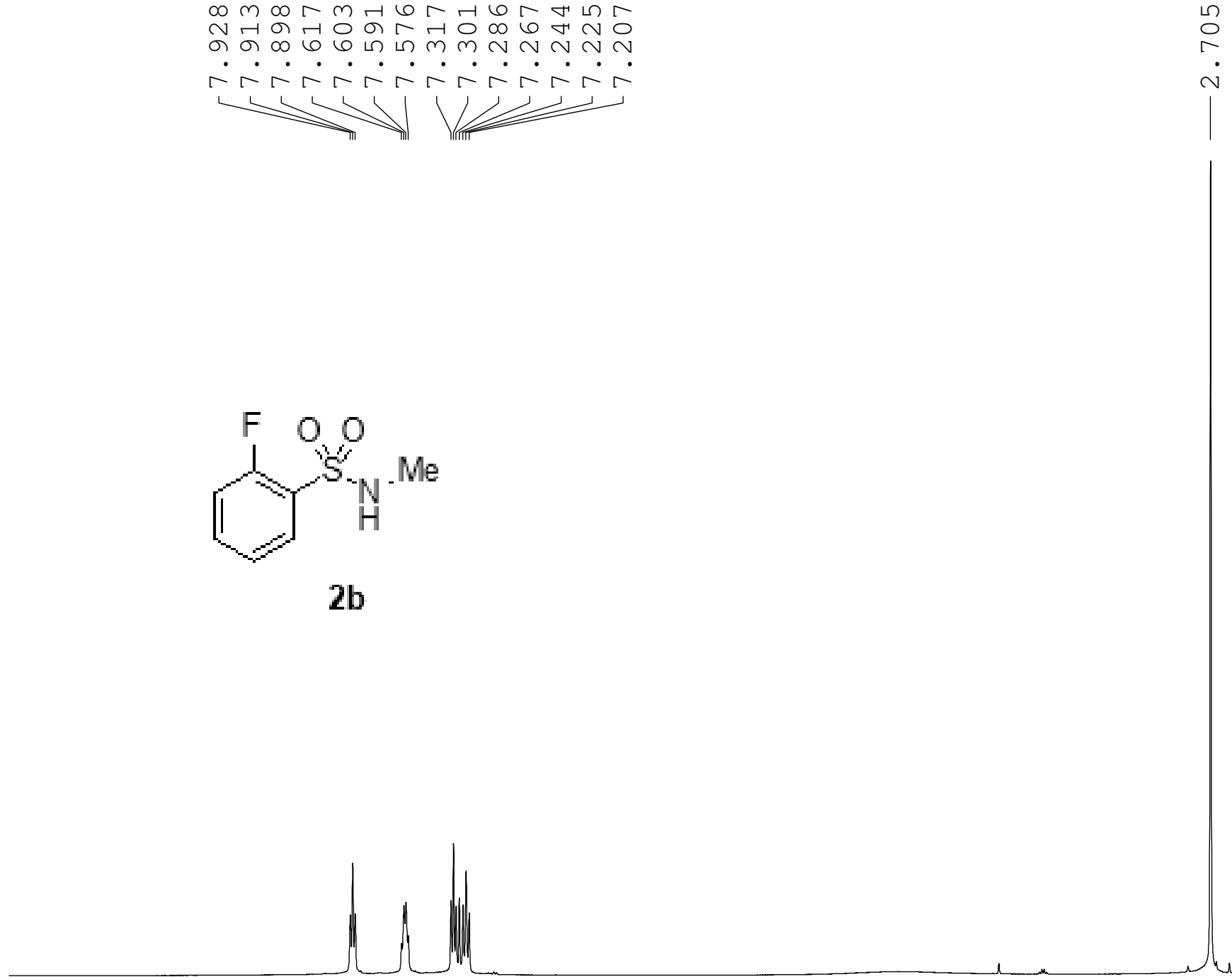

0
0
0
0
1

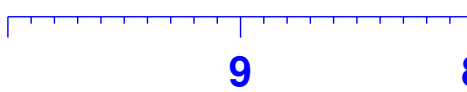

$$
\begin{aligned}
& 8 \\
& \text { (1) }
\end{aligned}
$$


2-fluoro-N-methylbenzenesulfonamide

C13CPD CDCl3
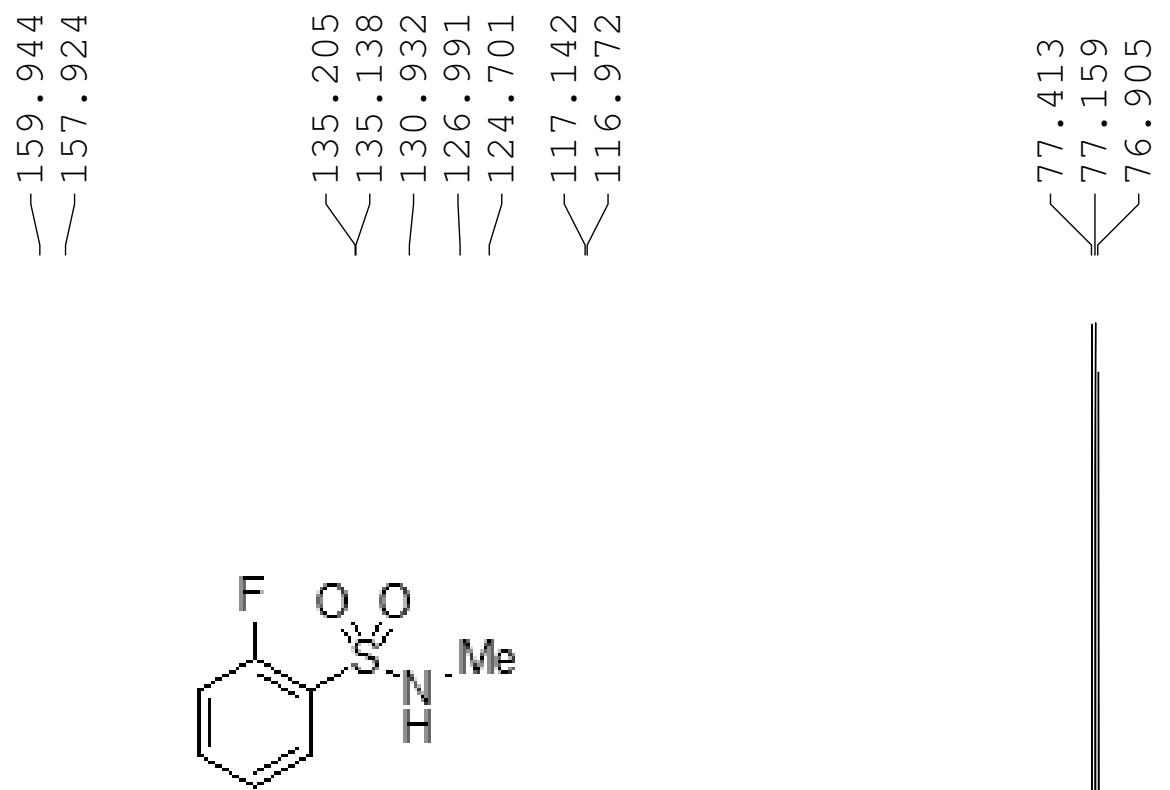

2b

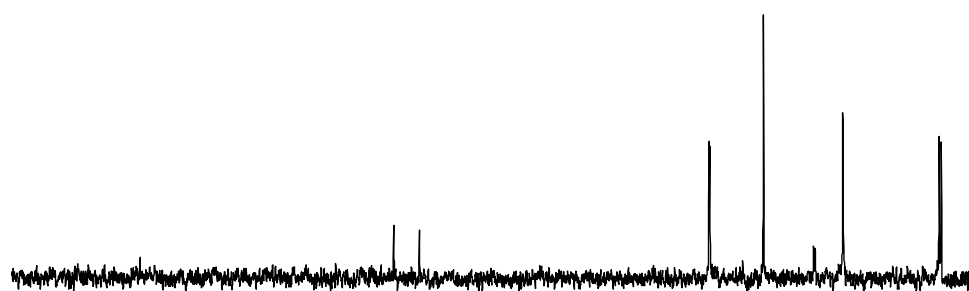

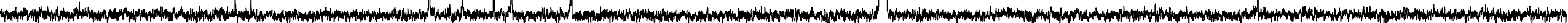


4-fluoro-N-methylbenzenesulfonamide Proton $\mathrm{CDCl} 3$
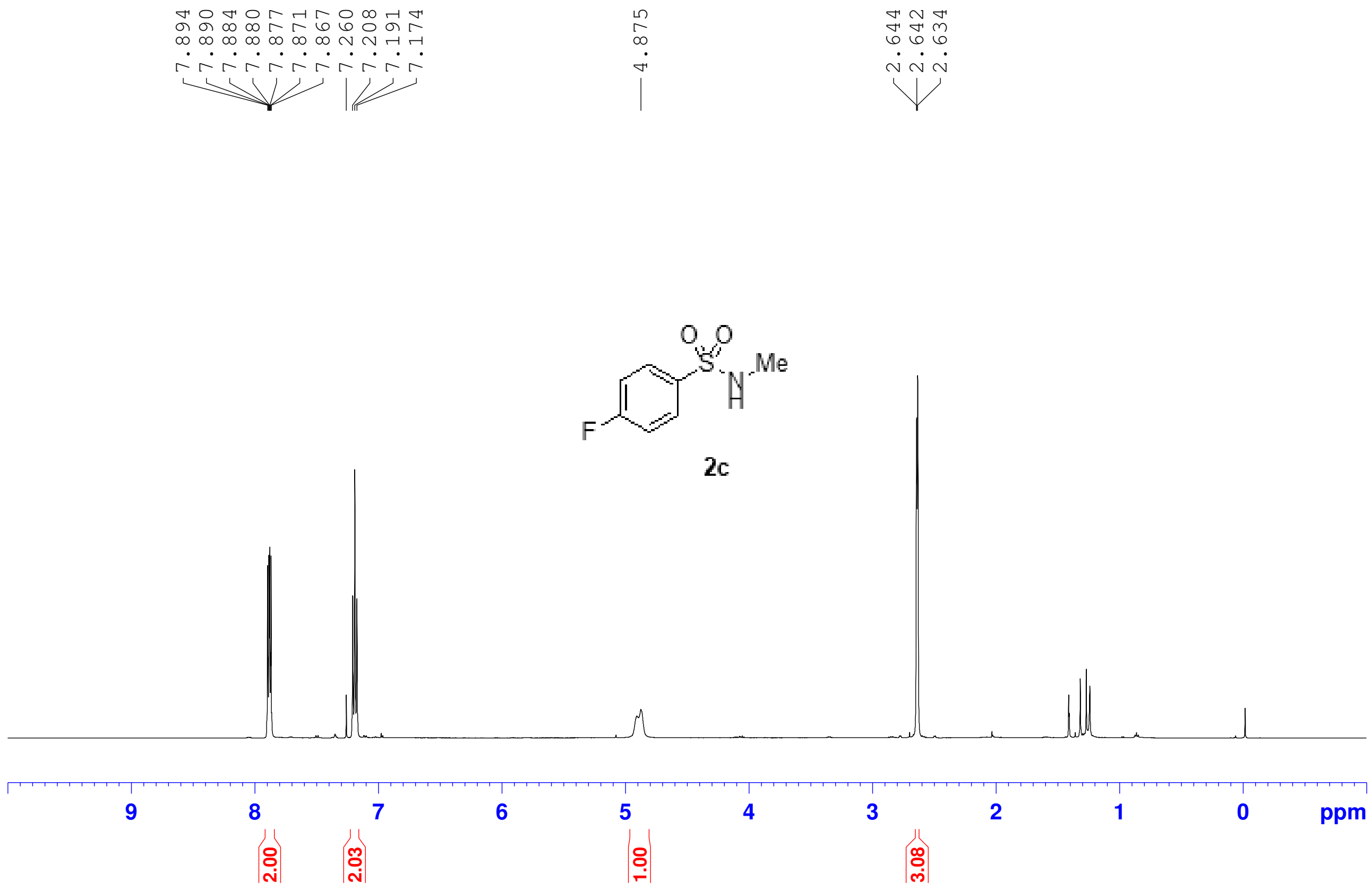
4-fluoro-N-methylbenzenesulfonamide

C13CPD CDCl3
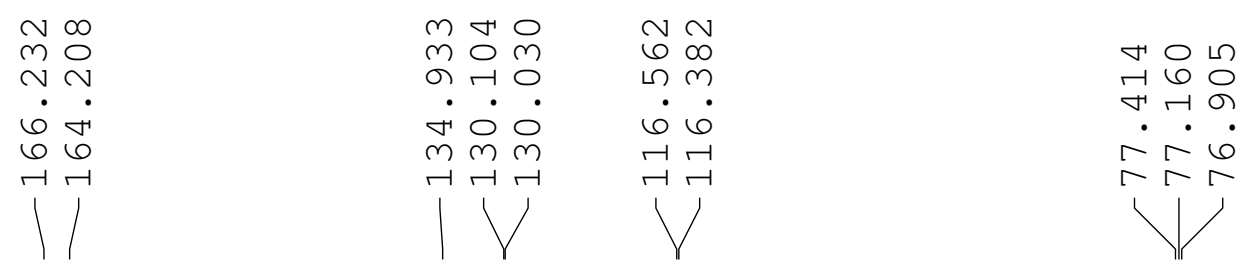

$\stackrel{\circ}{n}$
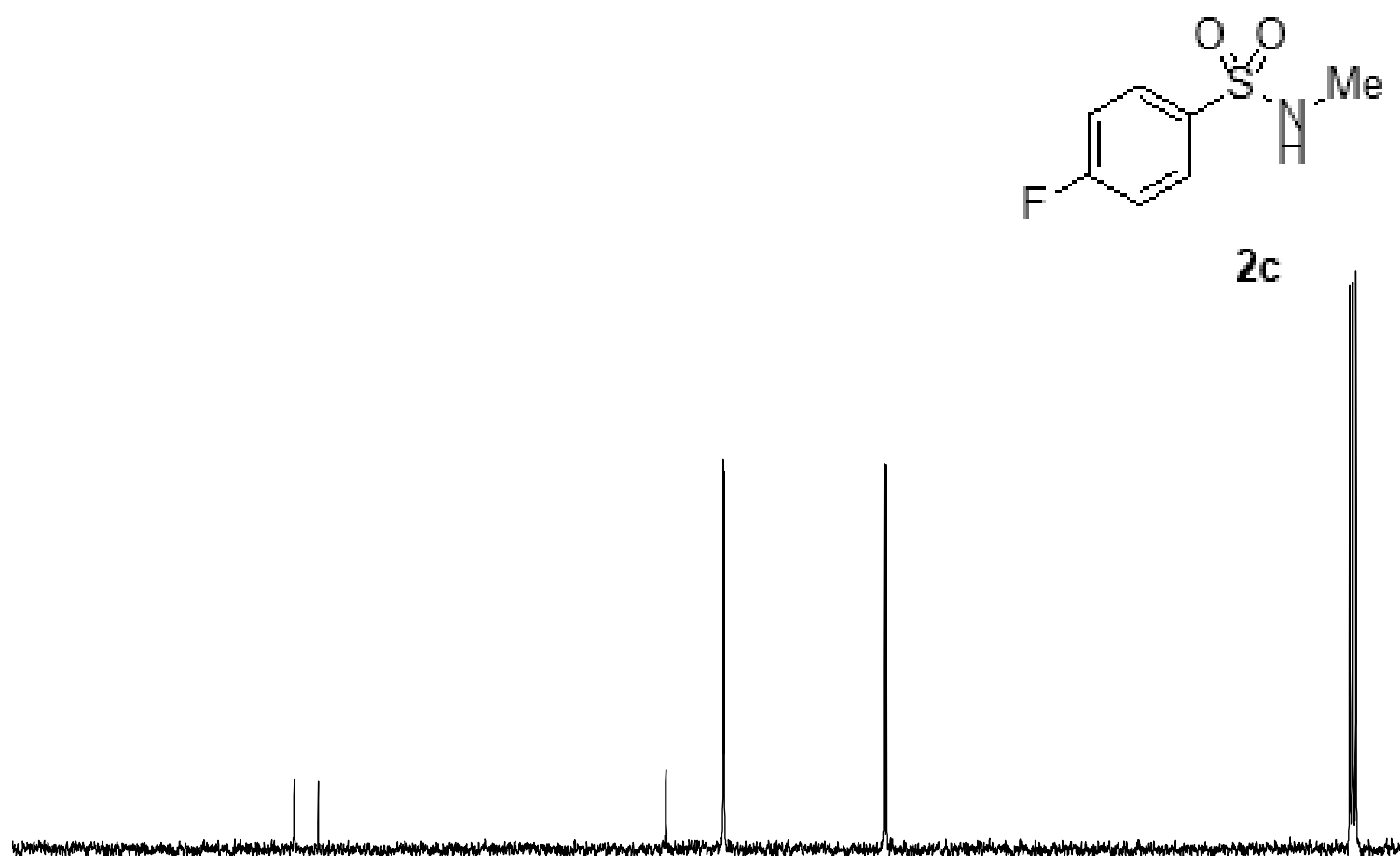

2c 
$\leftarrow \sim \sim \sim \curvearrowleft \circ m \in m \varphi 0$

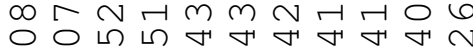

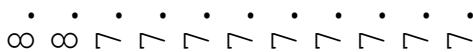

$\longrightarrow$
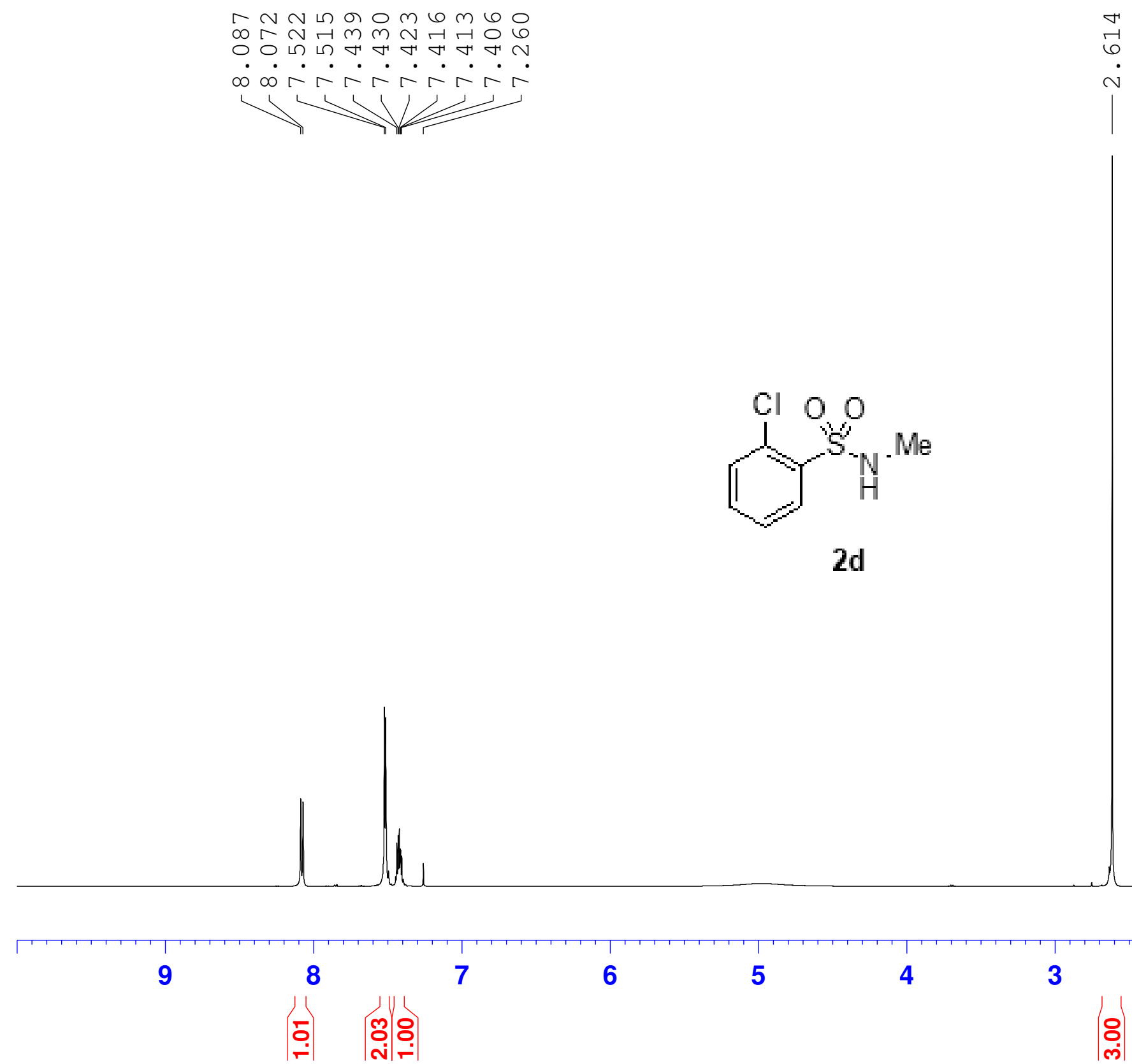
2-chloro-N-methylbenzenesulfonamide C13CPD CDCl3
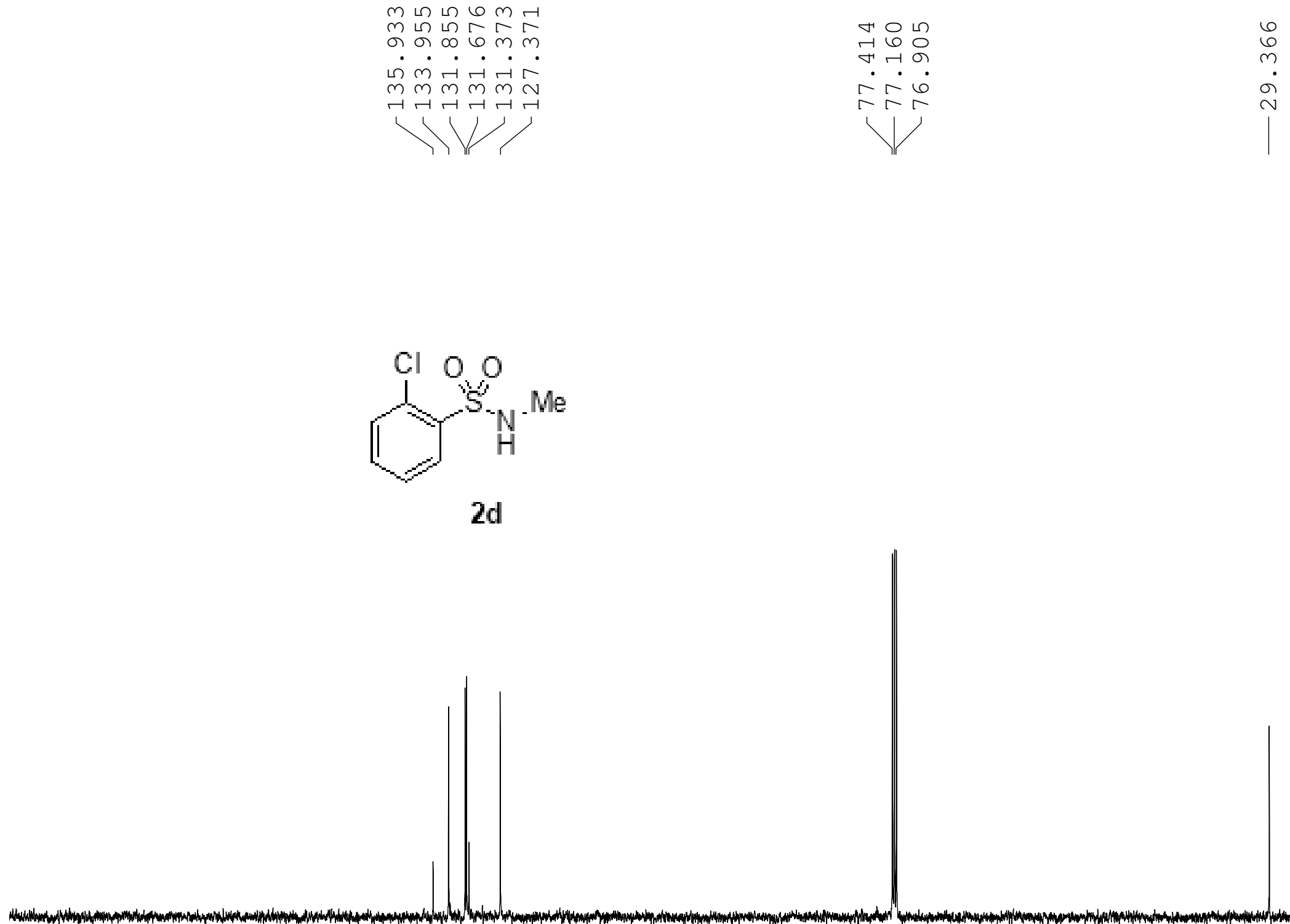
3-chloro-N-methylbenzenesulfonamide Proton $\mathrm{CDCl} 3$
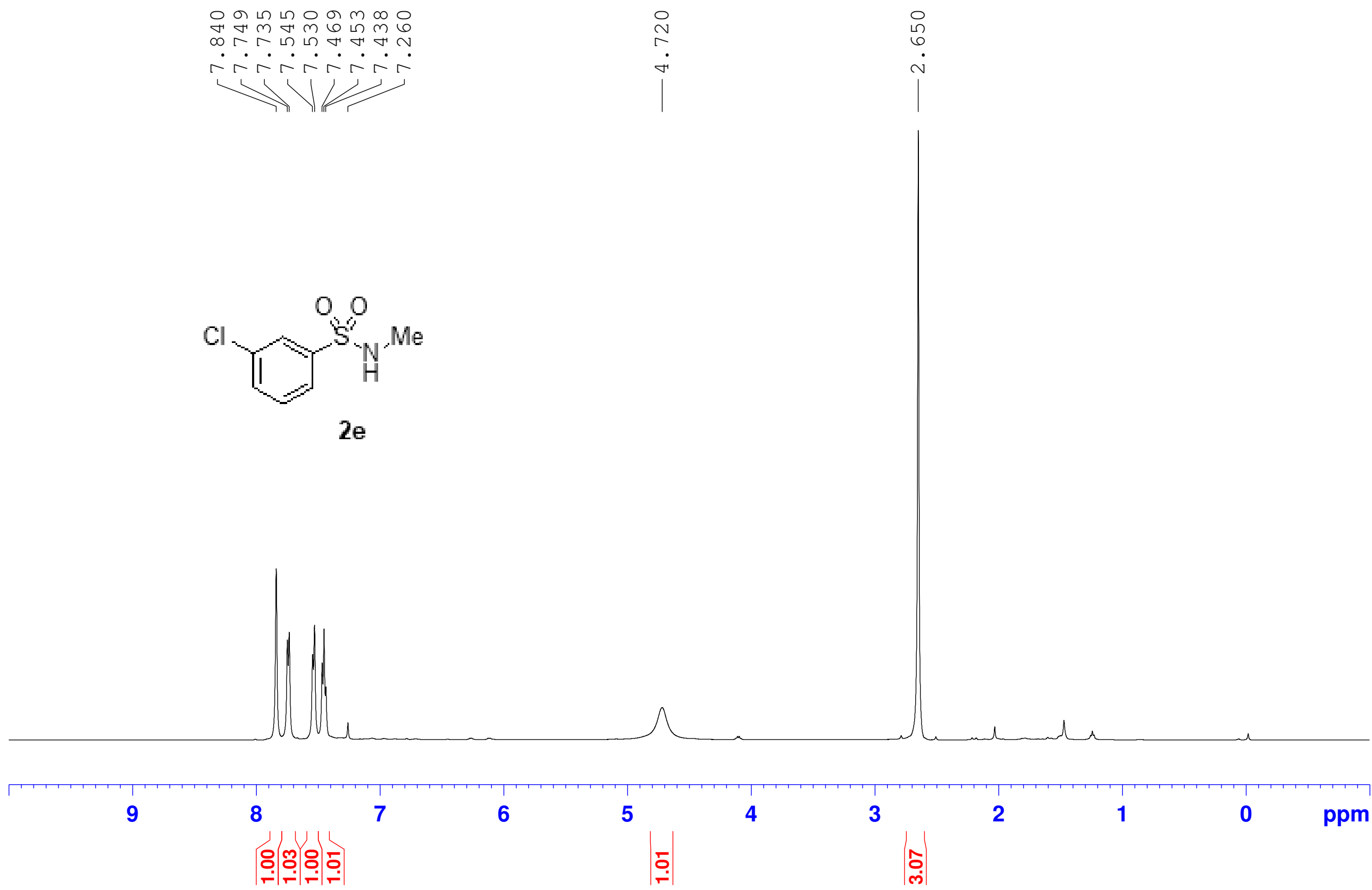
3-chloro-N-methylbenzenesulfonamide C13CPD CDCl3
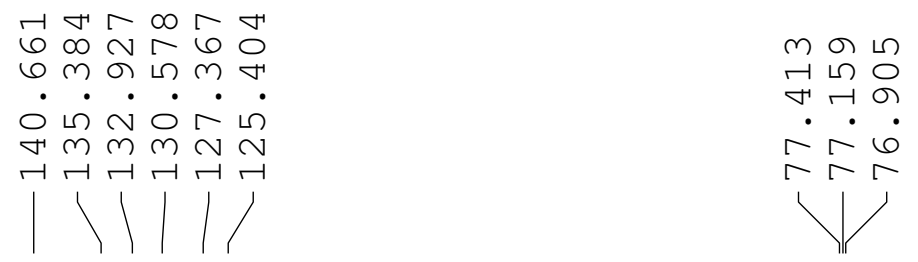

అై

on $\operatorname{con} 0$ in

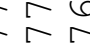

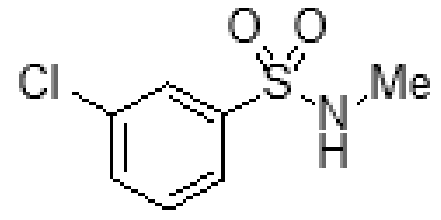

$2 e$

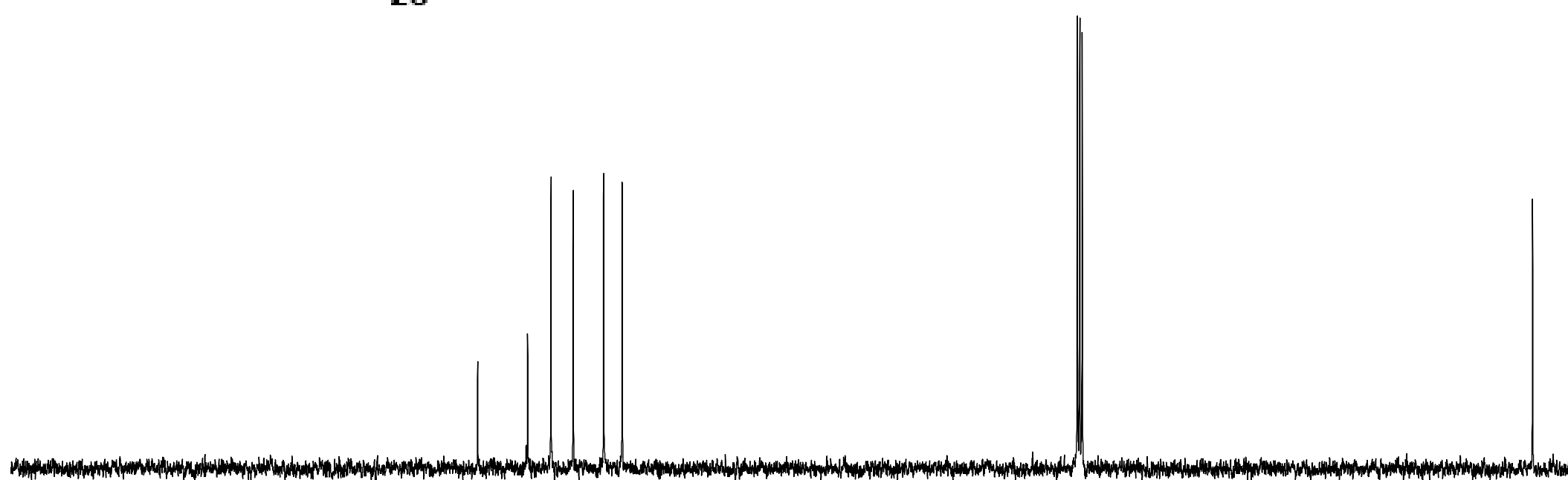

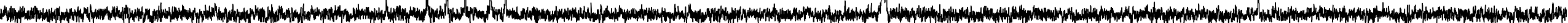


4-chloro-N-methylbenzenesulfonamide Proton $\mathrm{CDCl} 3$

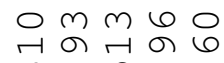

$$
\begin{aligned}
& \infty \pi \text { 制 } \\
& \text { R R R } \\
& 1 / 1
\end{aligned}
$$
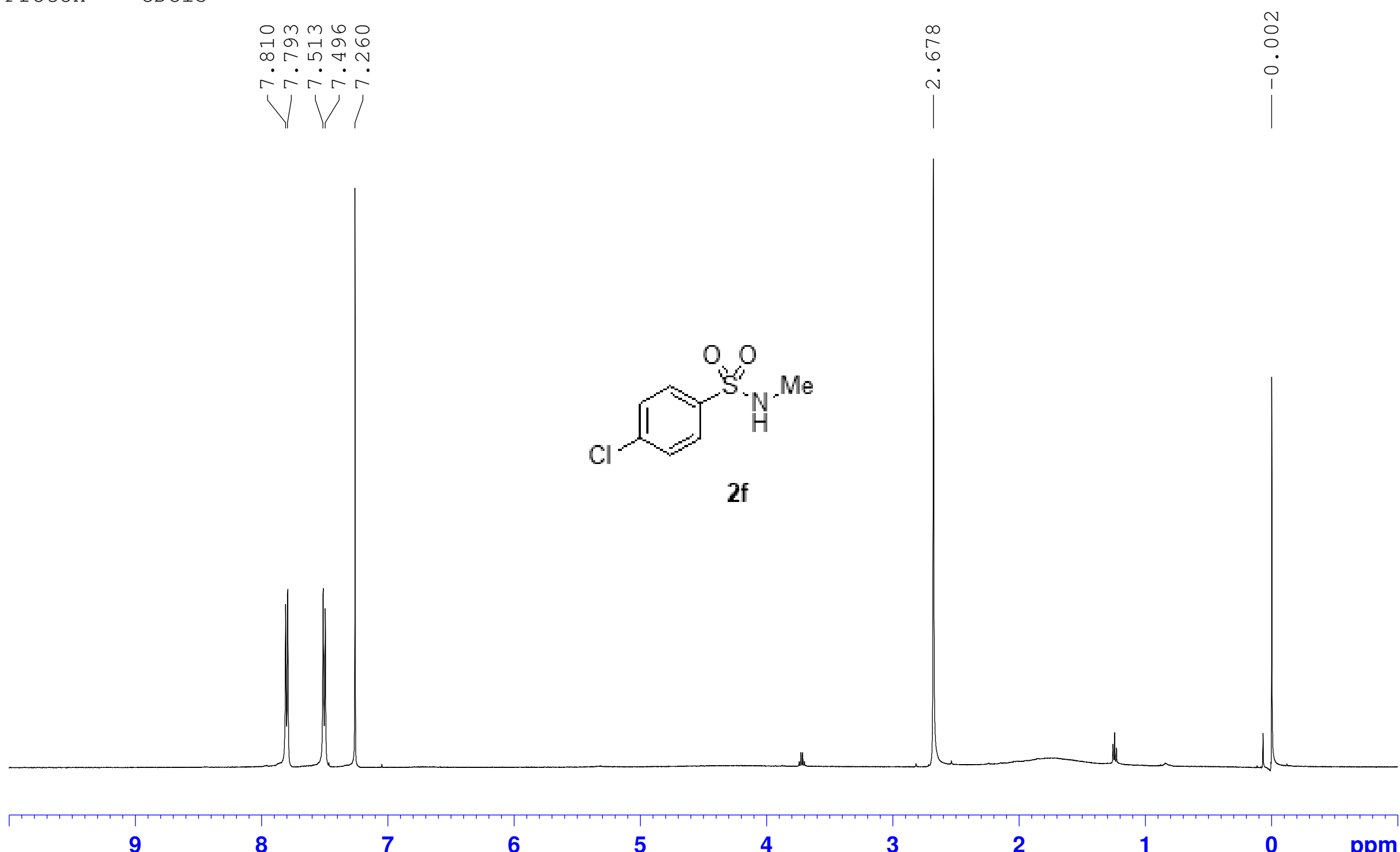

8

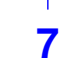

6

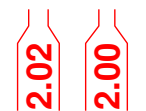

4

3

ल 
4-chloro-N-methylbenzenesulfonamide C13CPD CDCl3
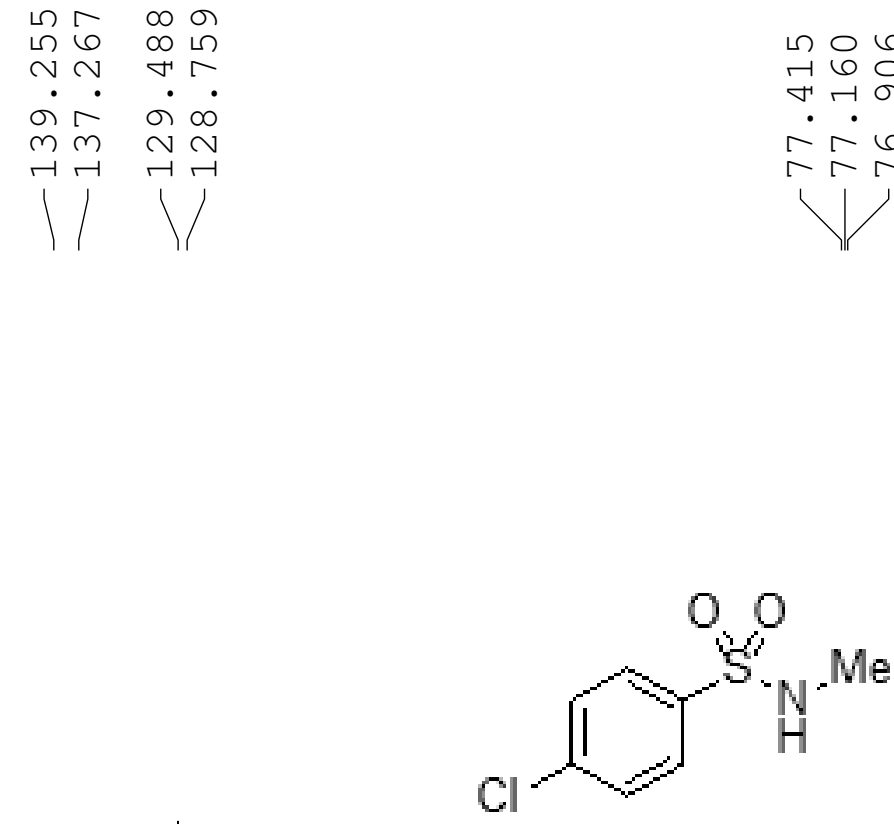

$2 f$

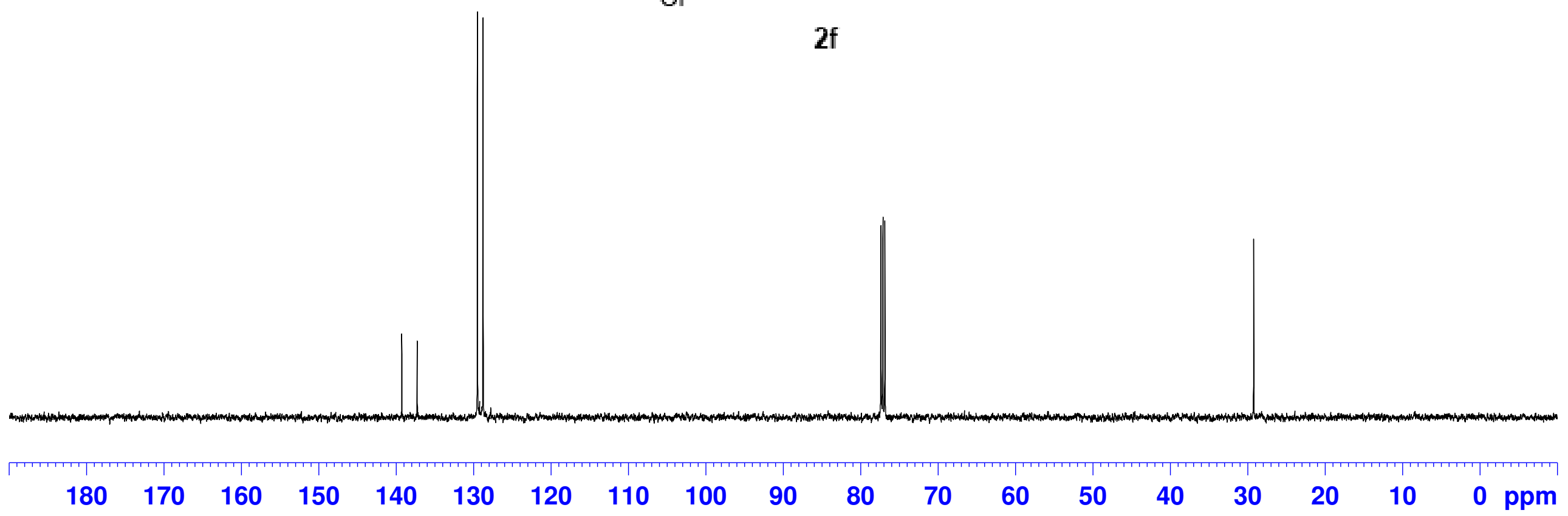


4-bromo-N-methylbenzenesulfonamide Proton CDCl3
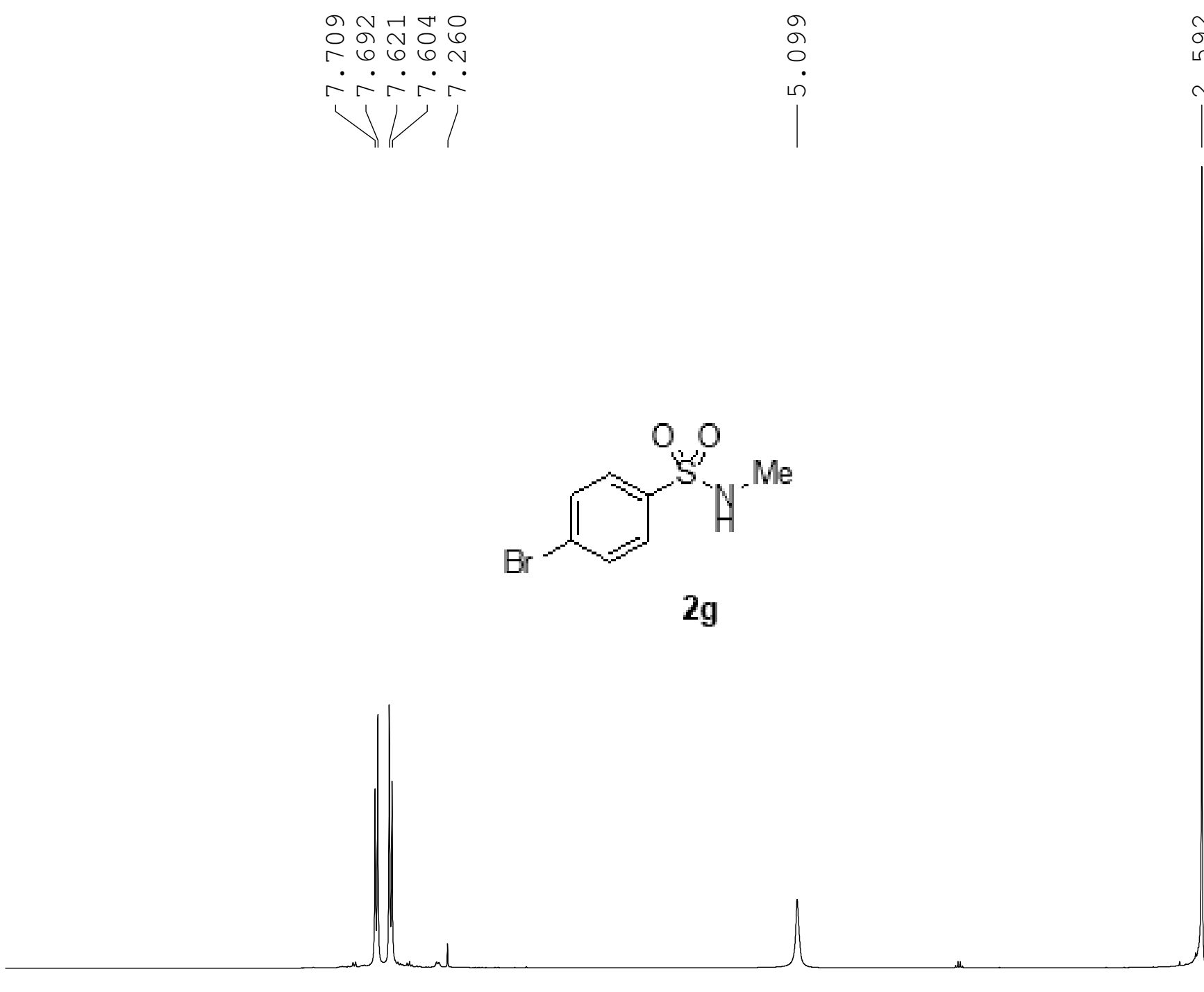
4-bromo-N-methylbenzenesulfonamide C13CPD CDCl3
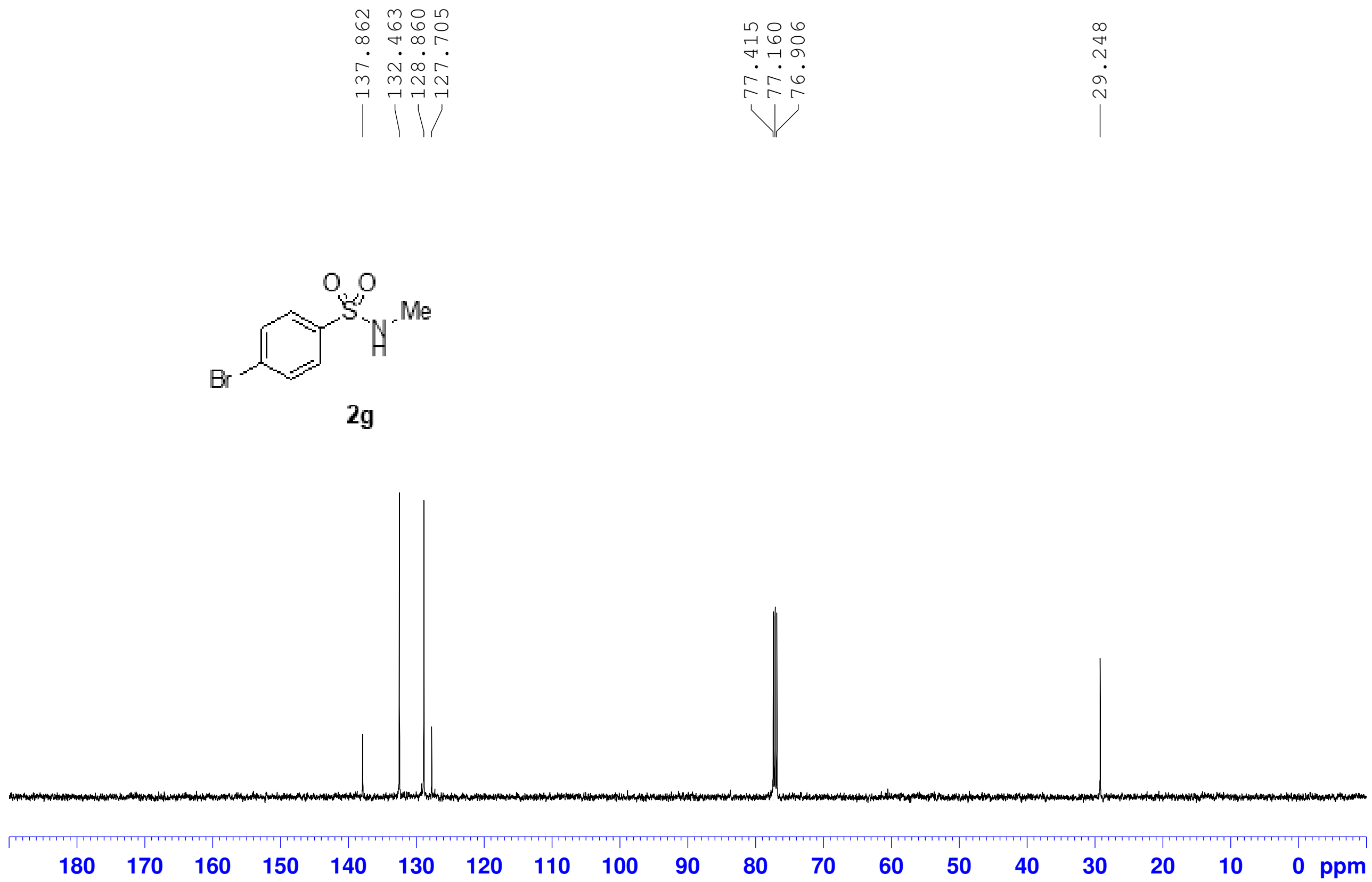
$\mathrm{N}$-methyl-4-(trifluoromethyl) benzenesulfonamide Proton CDCl3

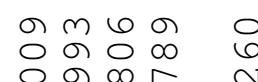

$$
\begin{aligned}
& \text { \% } \infty r \text {. } \\
& \infty \dot{\sim} \dot{\sim} \\
& \backslash 1
\end{aligned}
$$<smiles>CNS(=O)(=O)c1ccc(C(F)(F)F)cc1</smiles>

2h

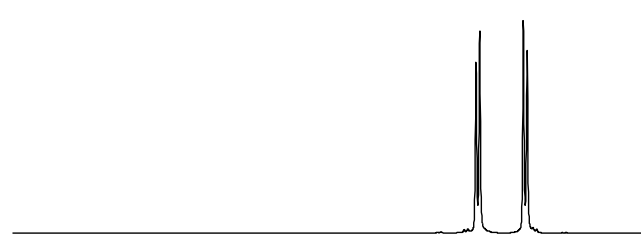

7

6

5

8.

(1) 
N-methyl-4-(trifluoromethyl) benzenesulfonamide C13CPD CDCl3

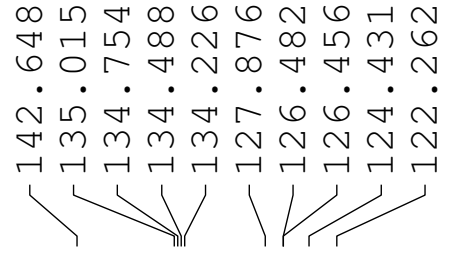<smiles>[M]NS(=O)(=O)c1ccc(C(F)(F)F)cc1</smiles>

$2 h$
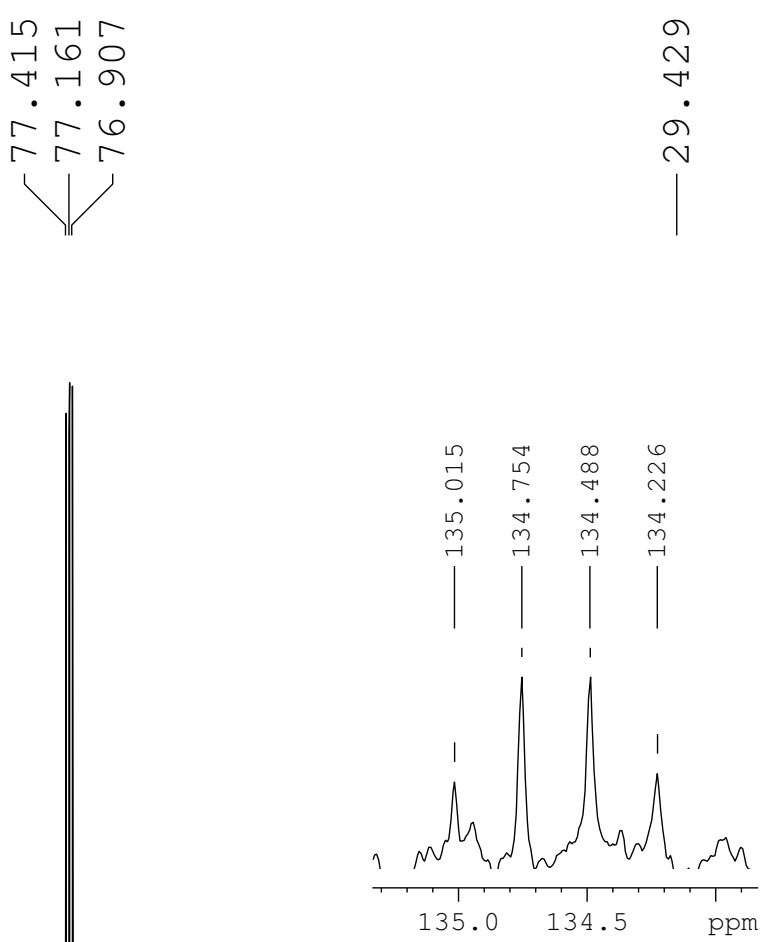

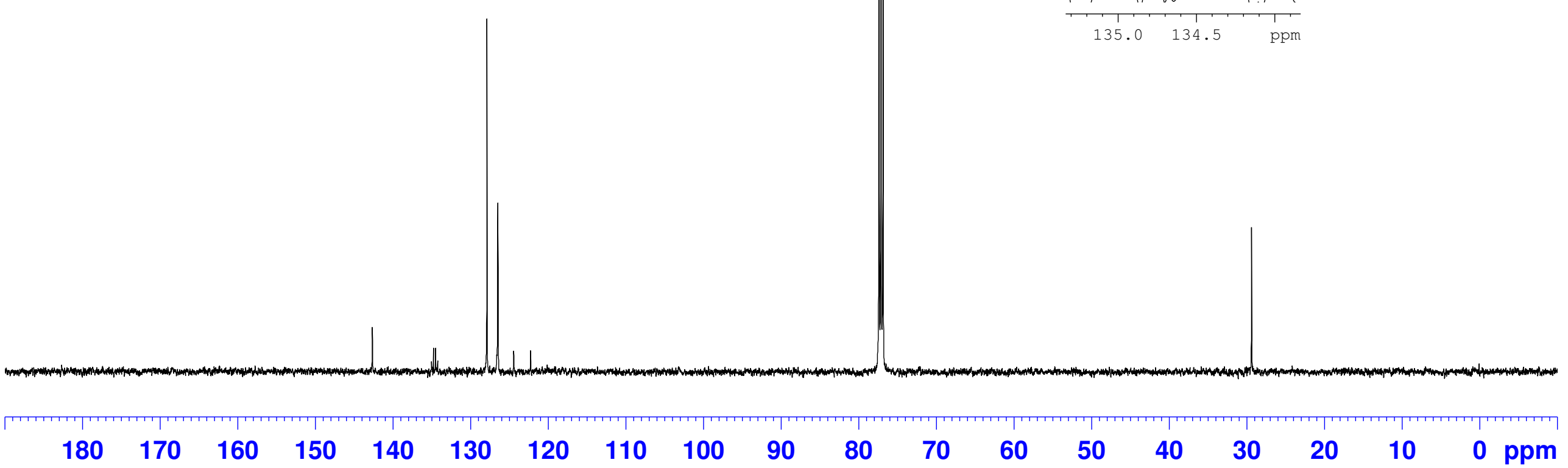


N-methyl-2-(trifluoromethyl) benzenesulfonamide Proton $\mathrm{CDCl} 3$
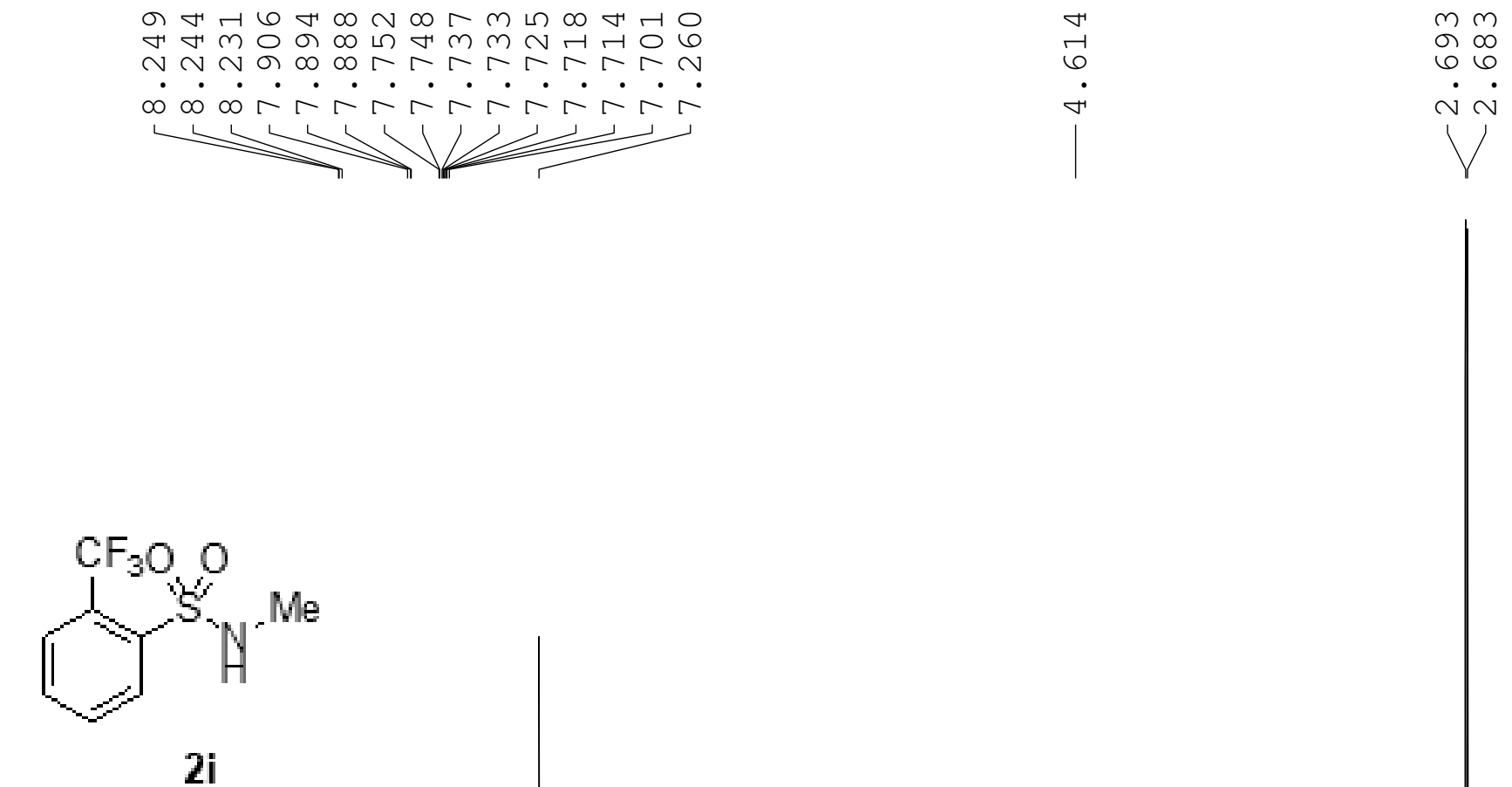

8

7

6

(ว) ชิ

5

4

3

$\left|\begin{array}{l}- \\ 0 \\ \text { m. }\end{array}\right|$ 
N-methyl-2-(trifluoromethyl) benzenesulfonamide C13CPD CDCl3
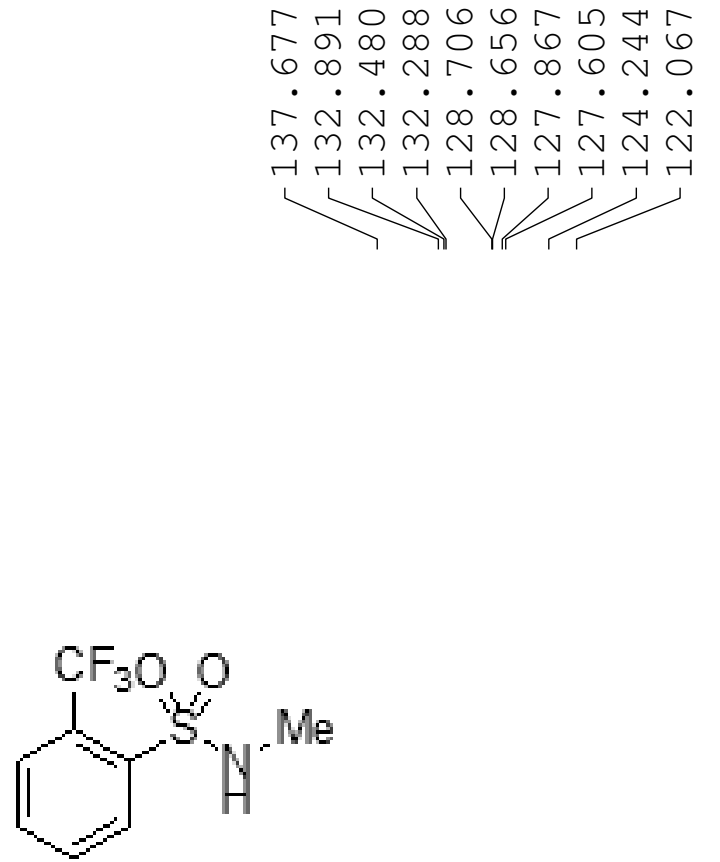

$2 \mathrm{i}$
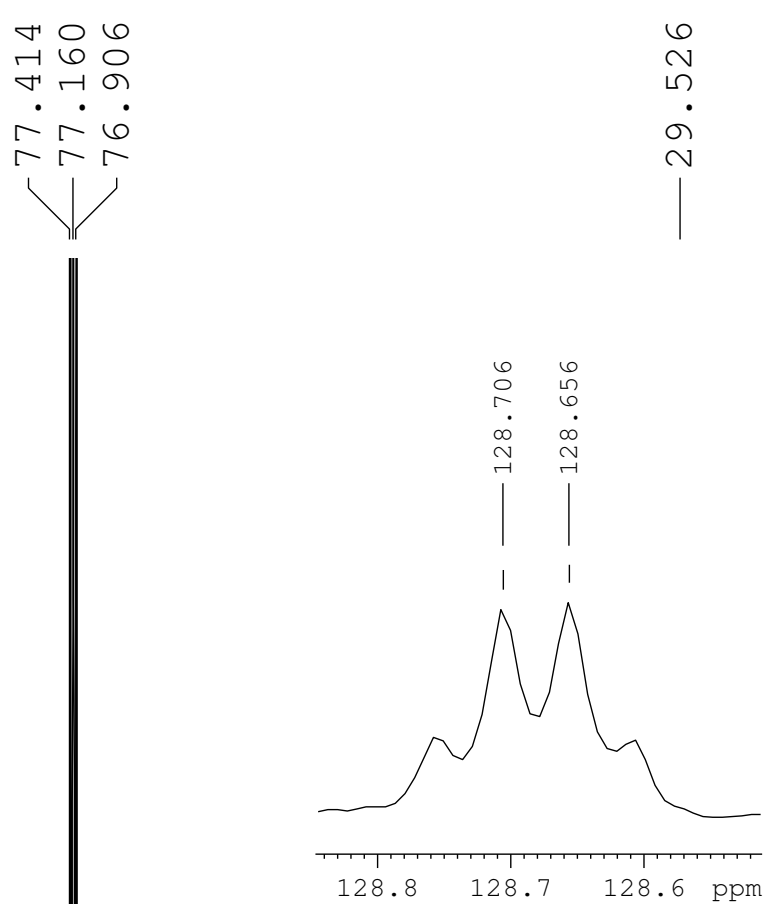

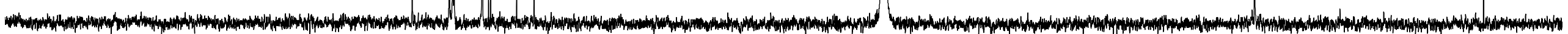
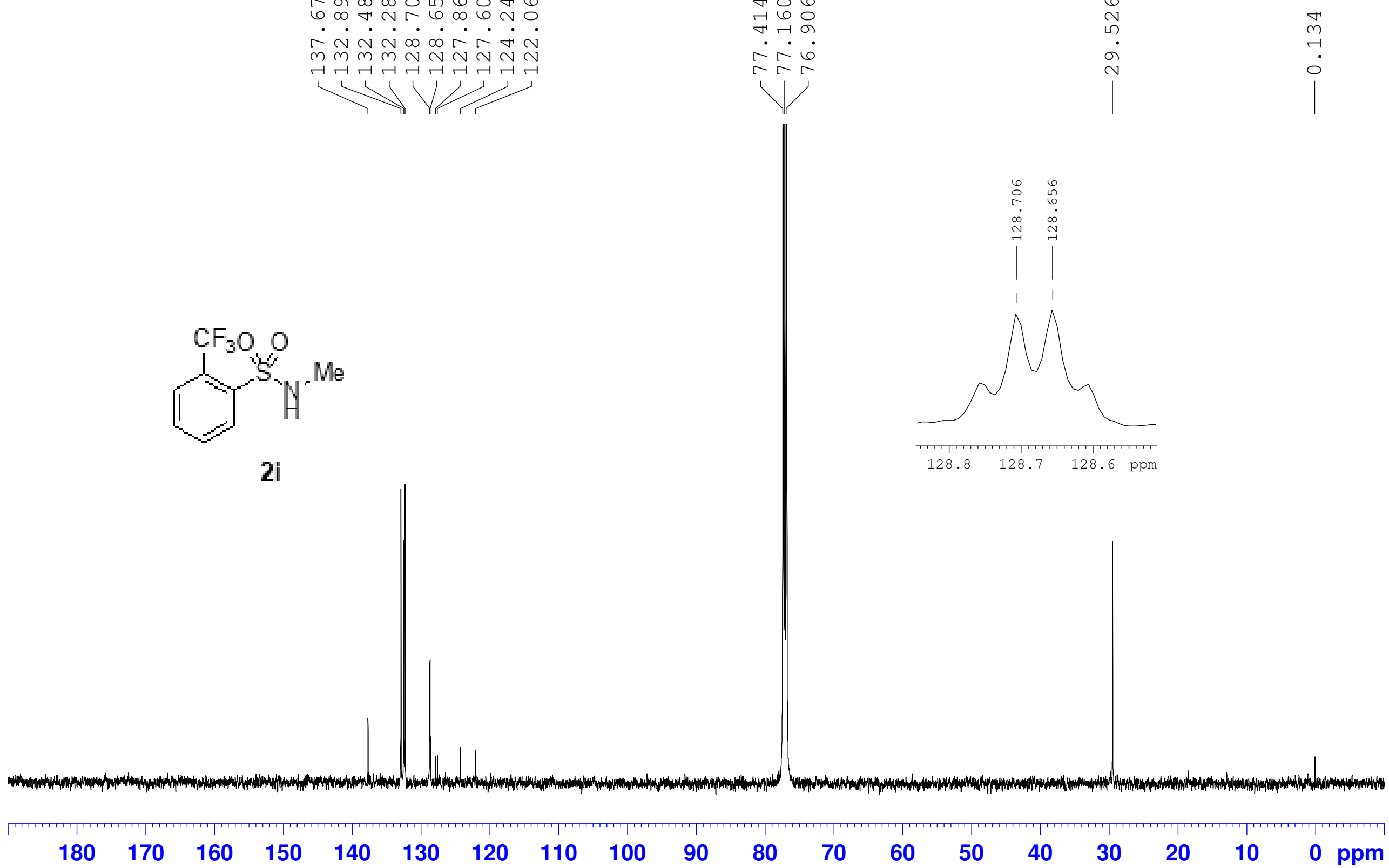
$\mathrm{N}$-methyl-4-(trifluoromethoxy) benzenesulfonamide Proton CDCl3

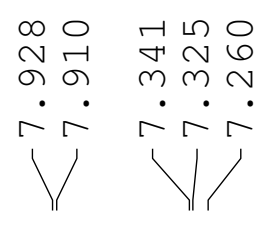

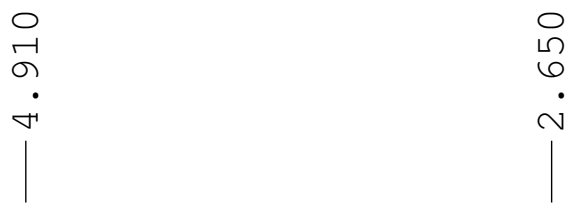
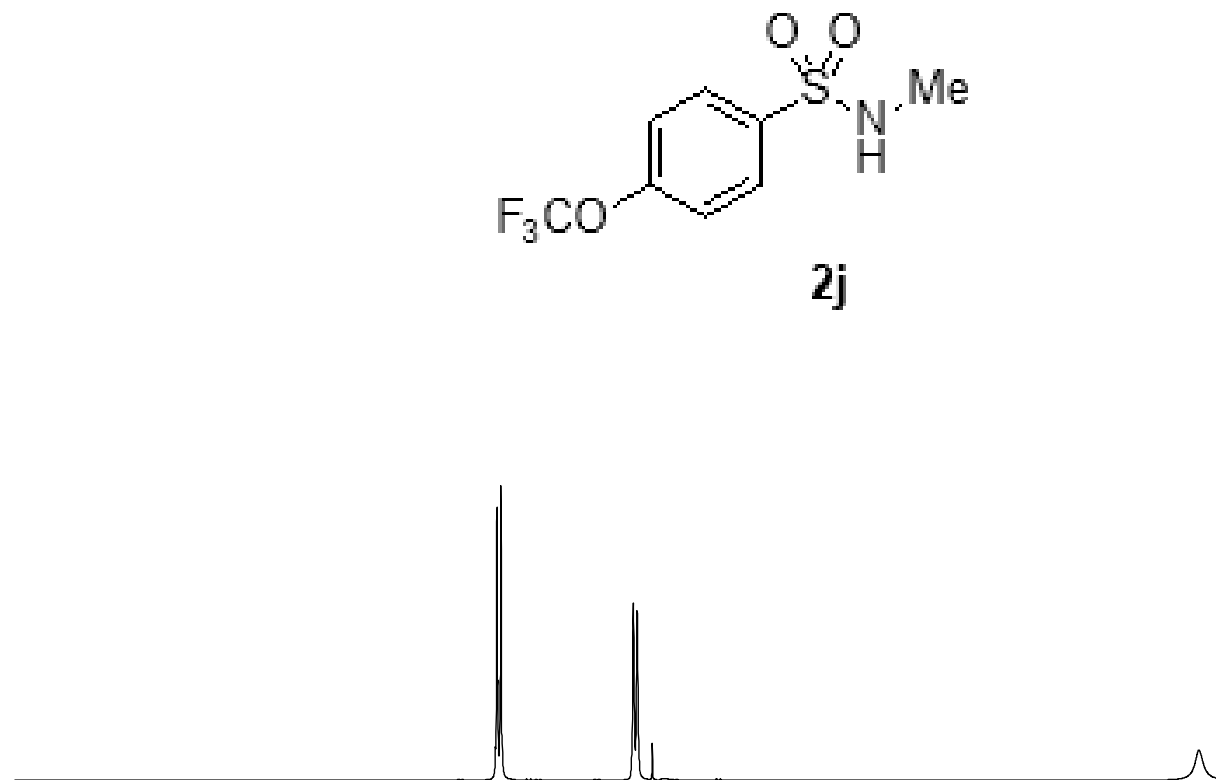

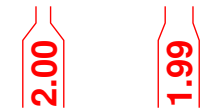


N-methyl-4-(trifluoromethoxy) benzenesulfonamide C13CPD CDCl3
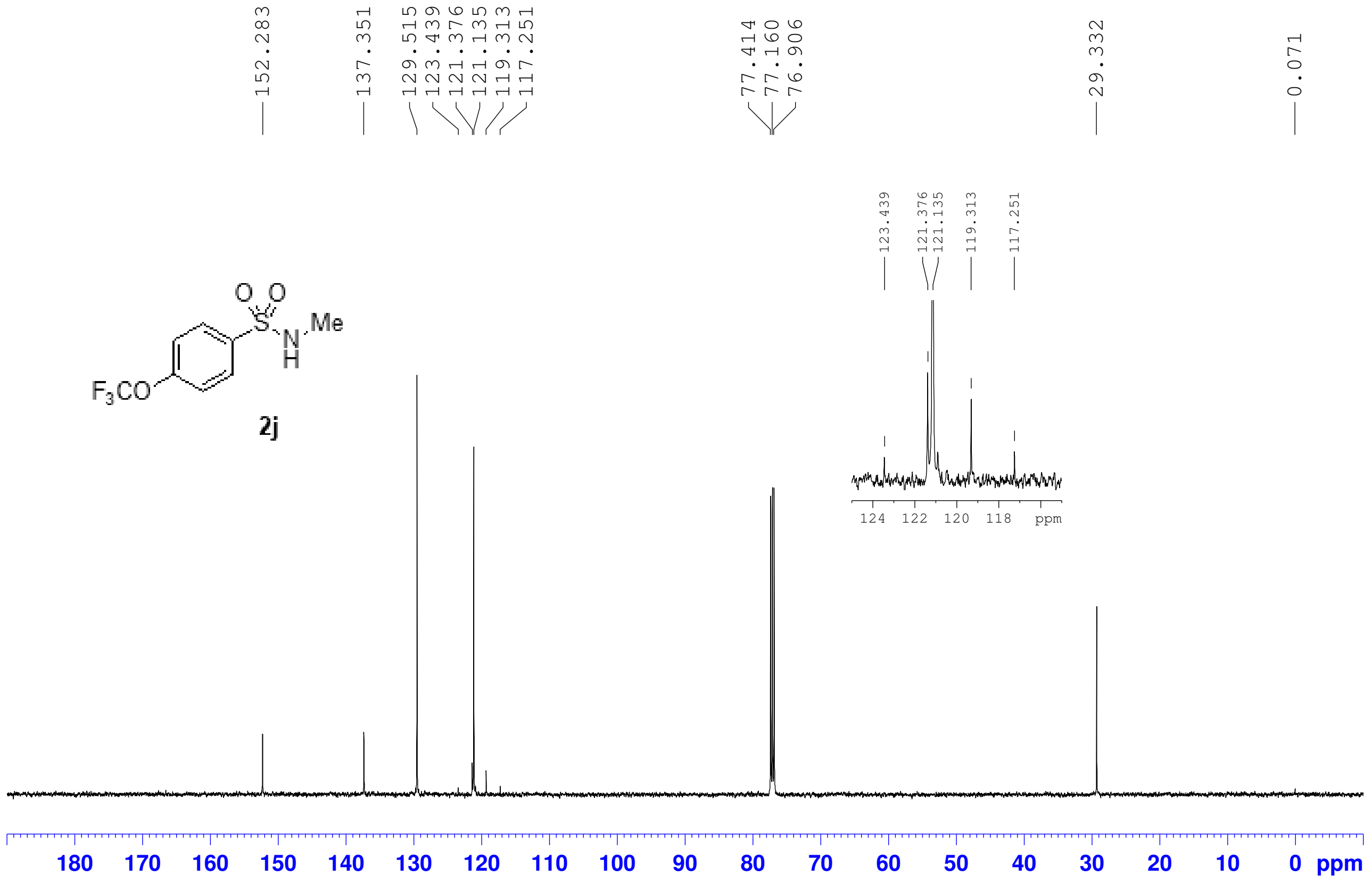
N, 2-dimethylbenzenesulfonamide Proton CDCl3

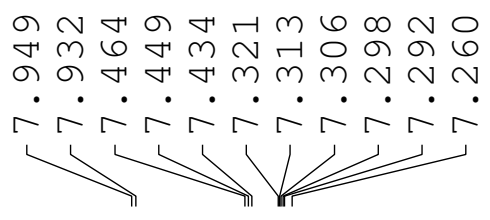

o

$\dot{\sim} \dot{\sim}$

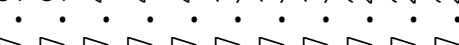

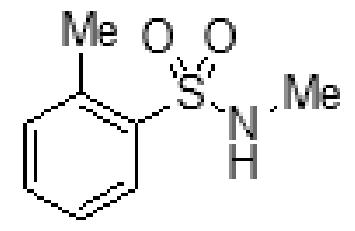

$2 k$

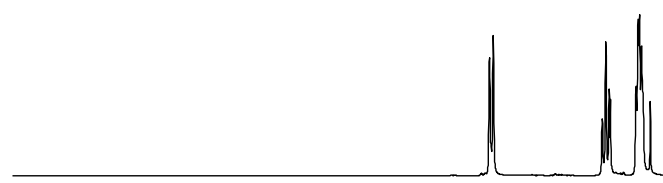

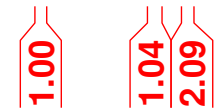


N, 2-dimethylbenzenesulfonamide

C13CPD CDCl3

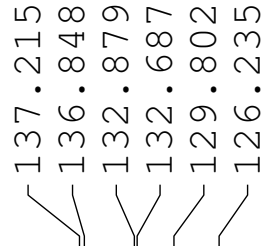

Me

$2 \mathrm{k}$
분용

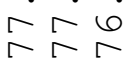

V

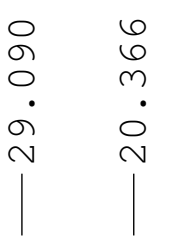

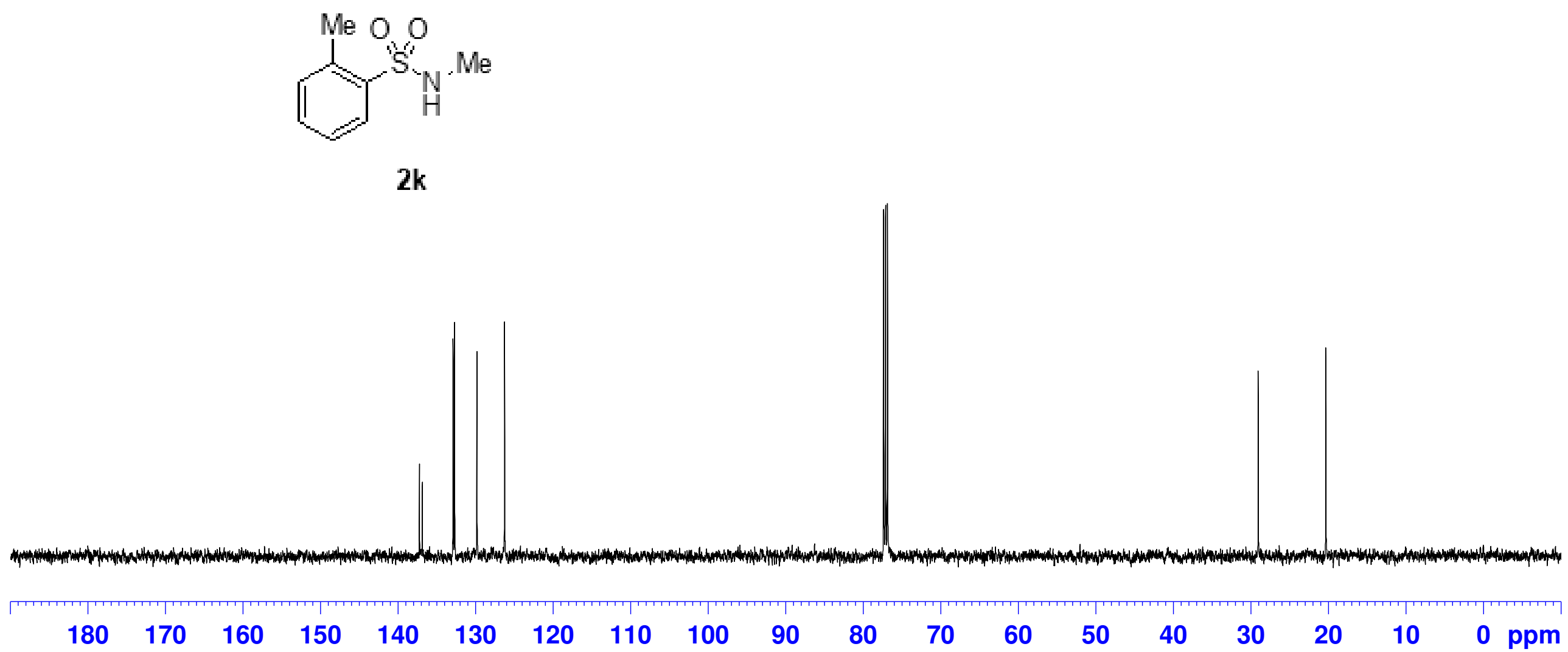


N, 4-dimethylbenzenesulfonamide Proton $\mathrm{CDCl} 3$
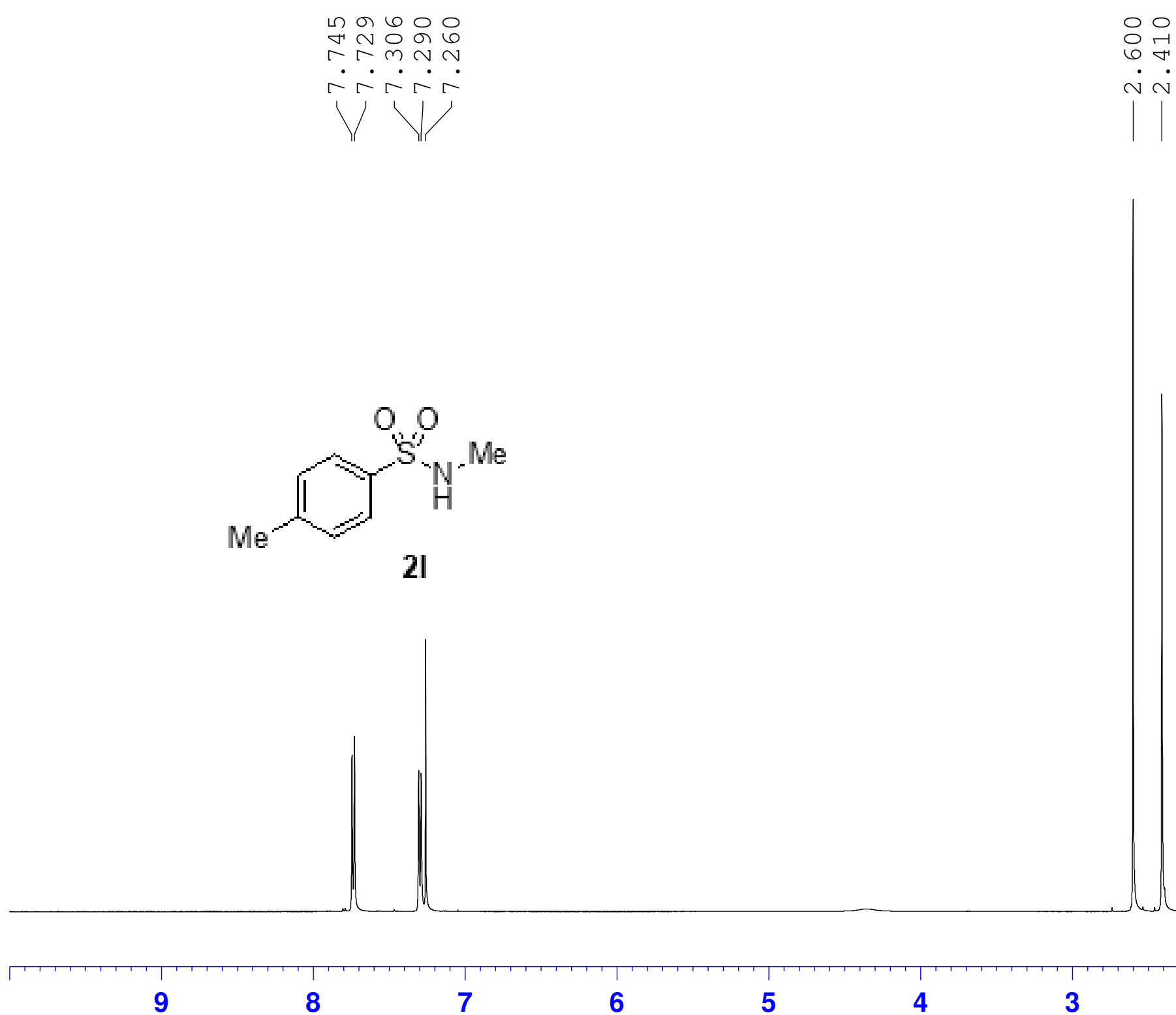

8 $\left|\begin{array}{c|c}5 & \\ 0 & 0 \\ 0 & 0 \\ \text { Ni }\end{array}\right|$

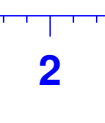

m. 
N, 4-dimethylbenzenesulfonamide C13CPD CDCl3
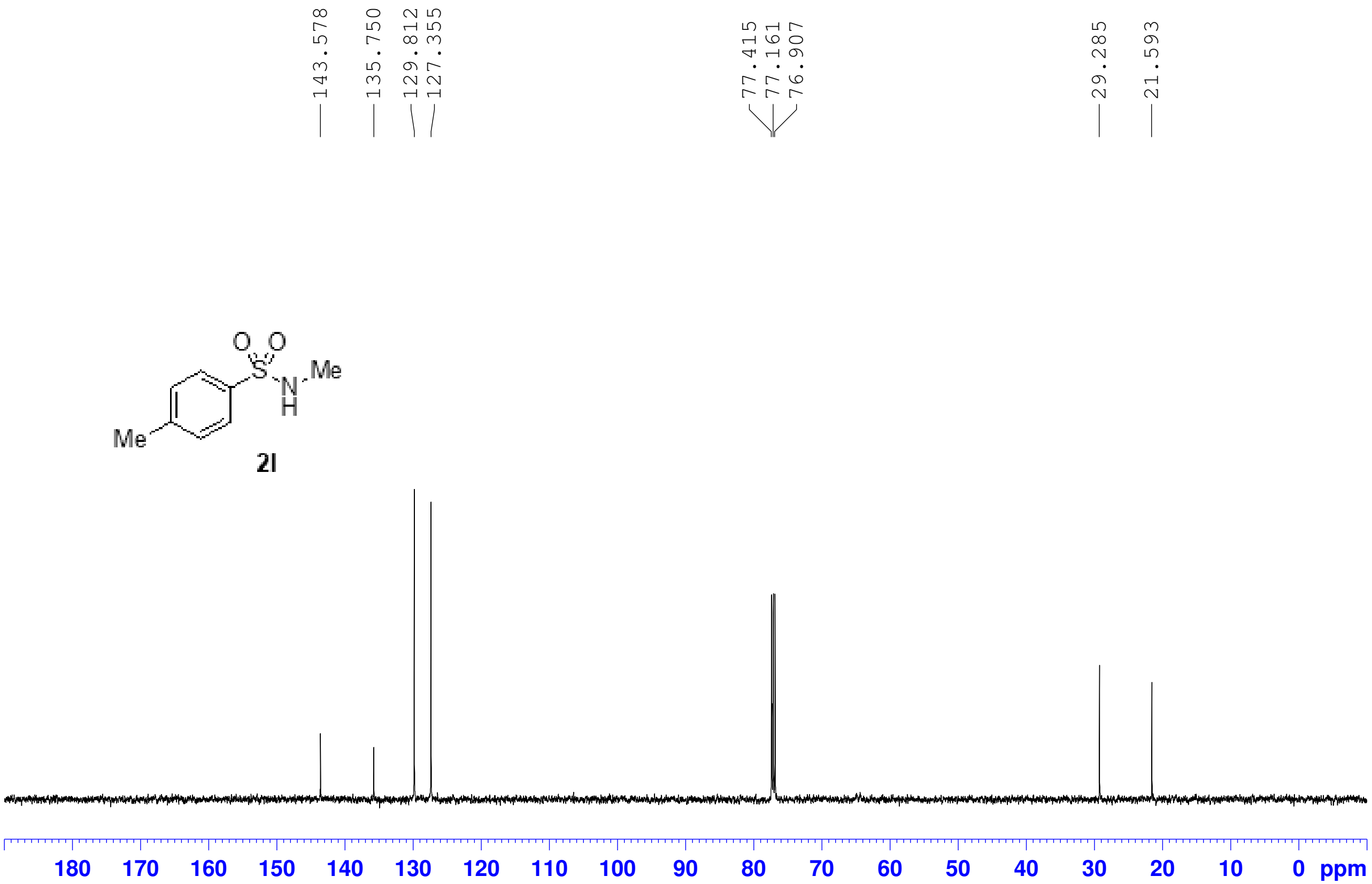
4-methoxy-N-methylbenzenesulfonamide Proton CDCl3
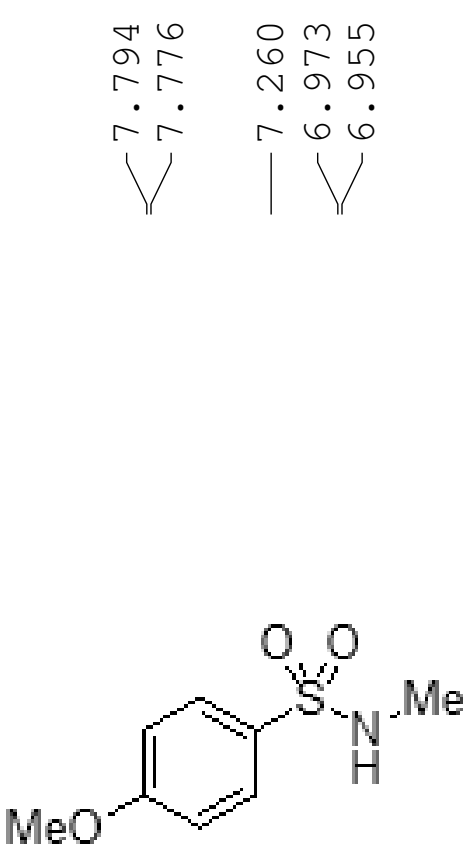

$2 m$

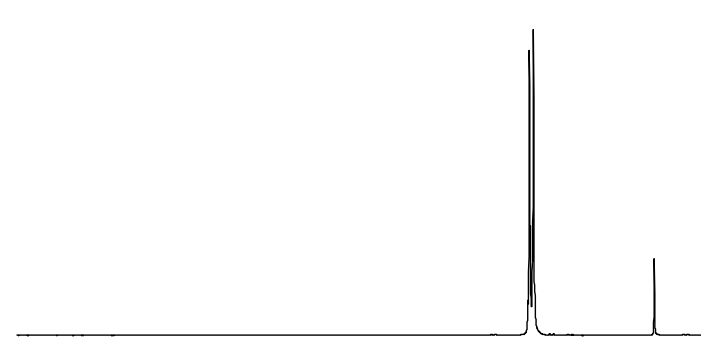

8

$\left|\begin{array}{l}\hat{O} \\ \mathbf{N} \\ \text { in }\end{array}\right|$
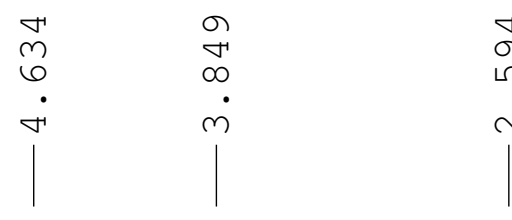

๙ิ

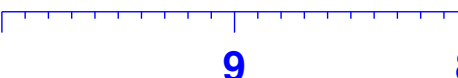

6

5

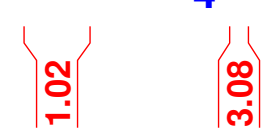

3

2

1

|ิ̀

ppm 
4-methoxy-N-methylbenzenesulfonamide C13CPD $\mathrm{CDCl} 3$

\begin{tabular}{|c|c|c|c|c|c|}
\hline$\sim$ & $r a$ & $\sim$ & & & \\
\hline$\infty$ & +6 & $\llcorner\cap$ & $\llcorner 0\llcorner$ & \llcorner & 6 \\
\hline or & m. & m. & 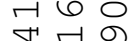 & ت & $m$ \\
\hline N & $\circ \sigma$ & $\sigma$ & . & - & \\
\hline 0 & $m \sim$ & 각 & r -6 & \llcorner & の \\
\hline & & 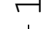 & $r-r$ & \llcorner & $\sim$ \\
\hline
\end{tabular}

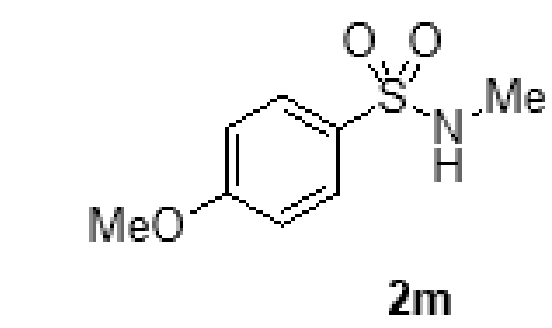

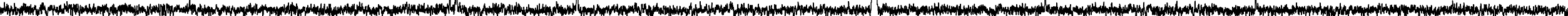

$\begin{array}{llllllllllllllllllll}180 & 170 & 160 & 150 & 140 & 130 & 120 & 110 & 100 & 90 & 80 & 70 & 60 & 50 & 40 & 30 & 20 & 10 & 0 & \mathrm{ppm}\end{array}$


N-methylnaphthalene-2-sulfonamide Proton CDCl3

๙ अ maririririririr

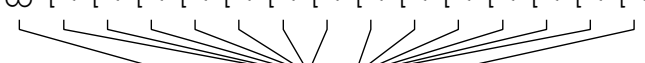
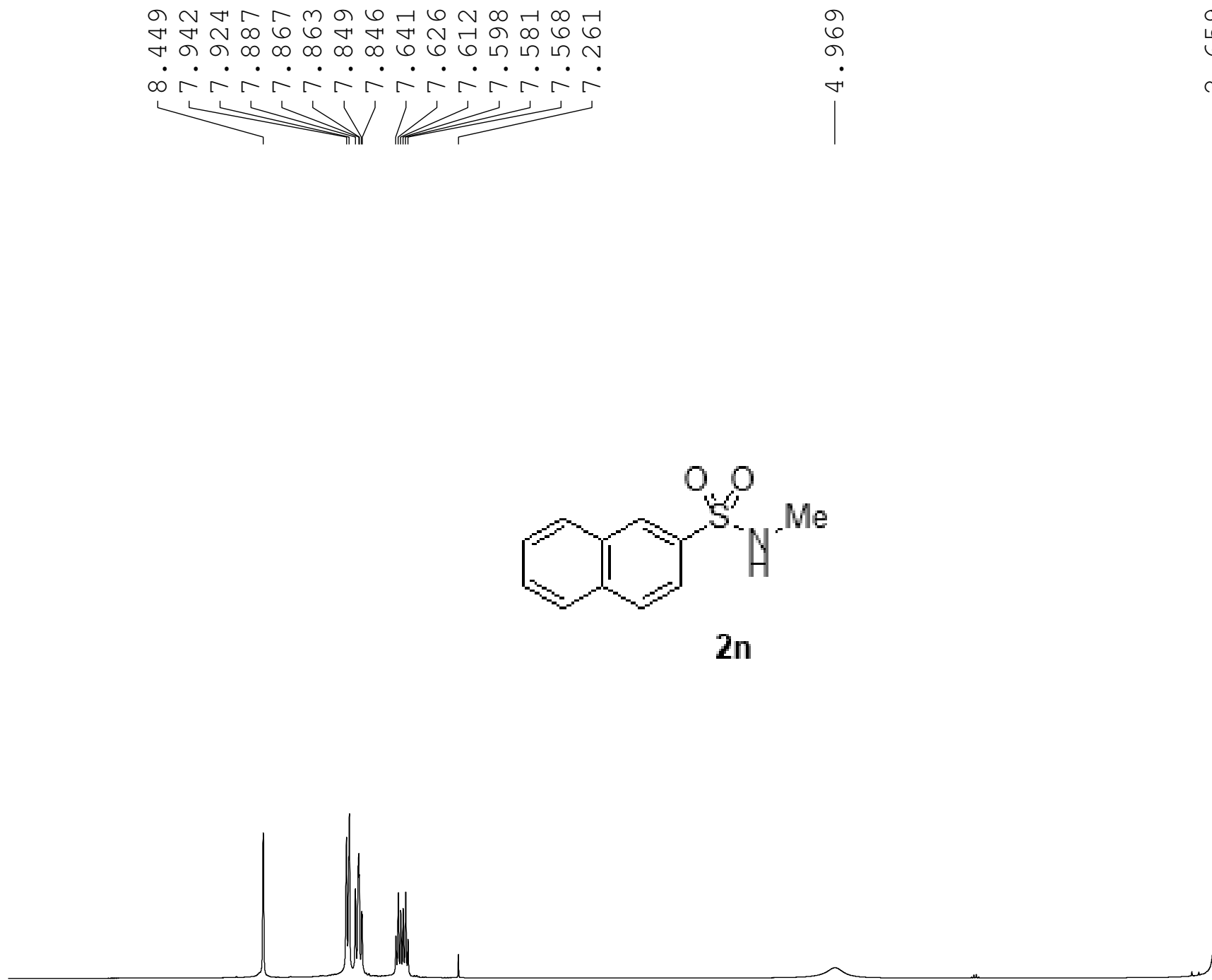

8

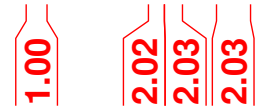

7

6

ำ

4

3

2

1

$\left|\begin{array}{c}\delta \\ \text { m. }\end{array}\right|$ 
$\mathrm{N}$-methylnaphthalene-2-sulfonamide

C13CPD CDCl3

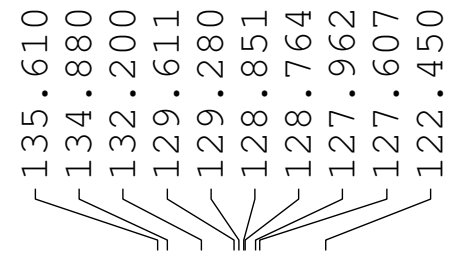

ㄷํㅁ

-

1

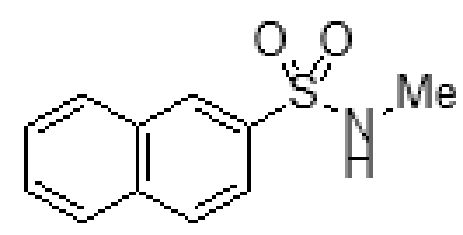

2n
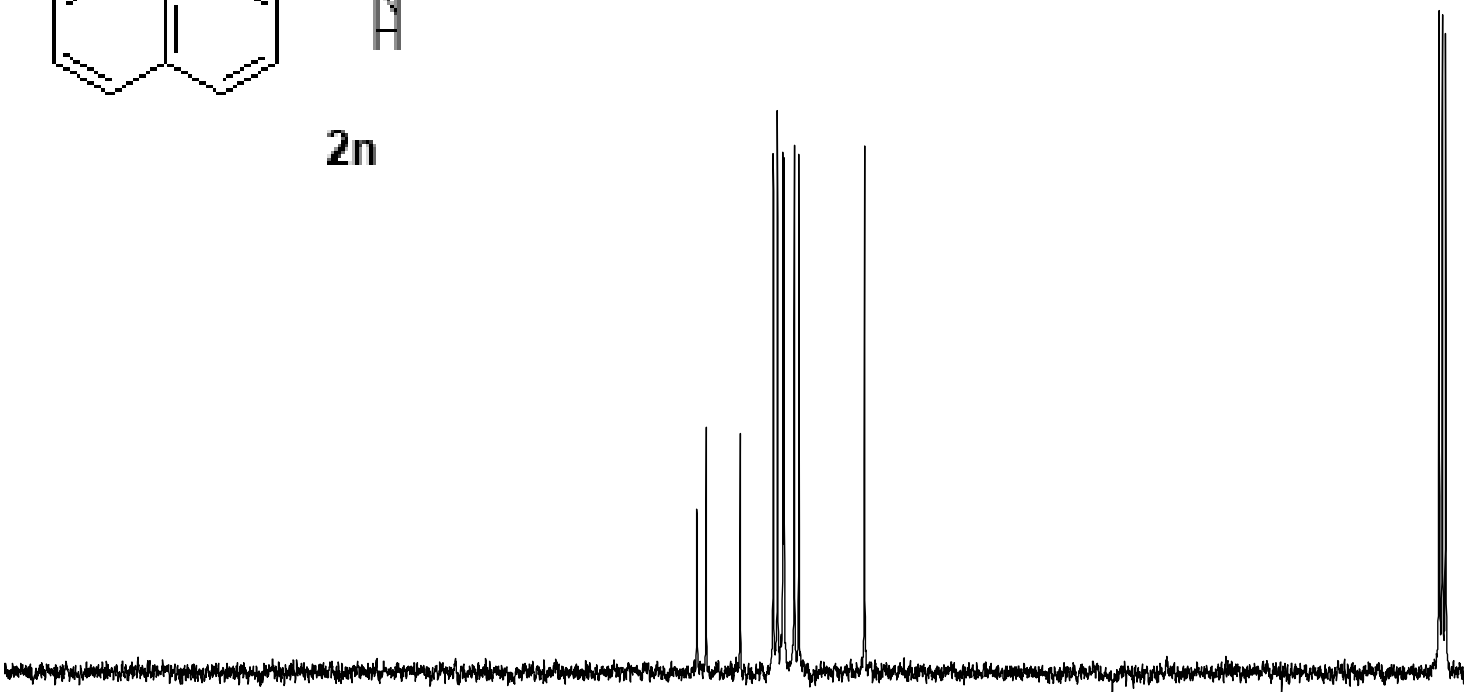

180

170160

150

140

130

120

110

100

90

80

70

60

50

40

30

20

0 ppm 
$\mathrm{N}$-methyl (phenyl) methanesulfonamide Proton CDCl3

$$
\begin{array}{ll}
N & 0 \\
\infty & 6 \\
m & \cdots \\
1 & 1
\end{array}
$$
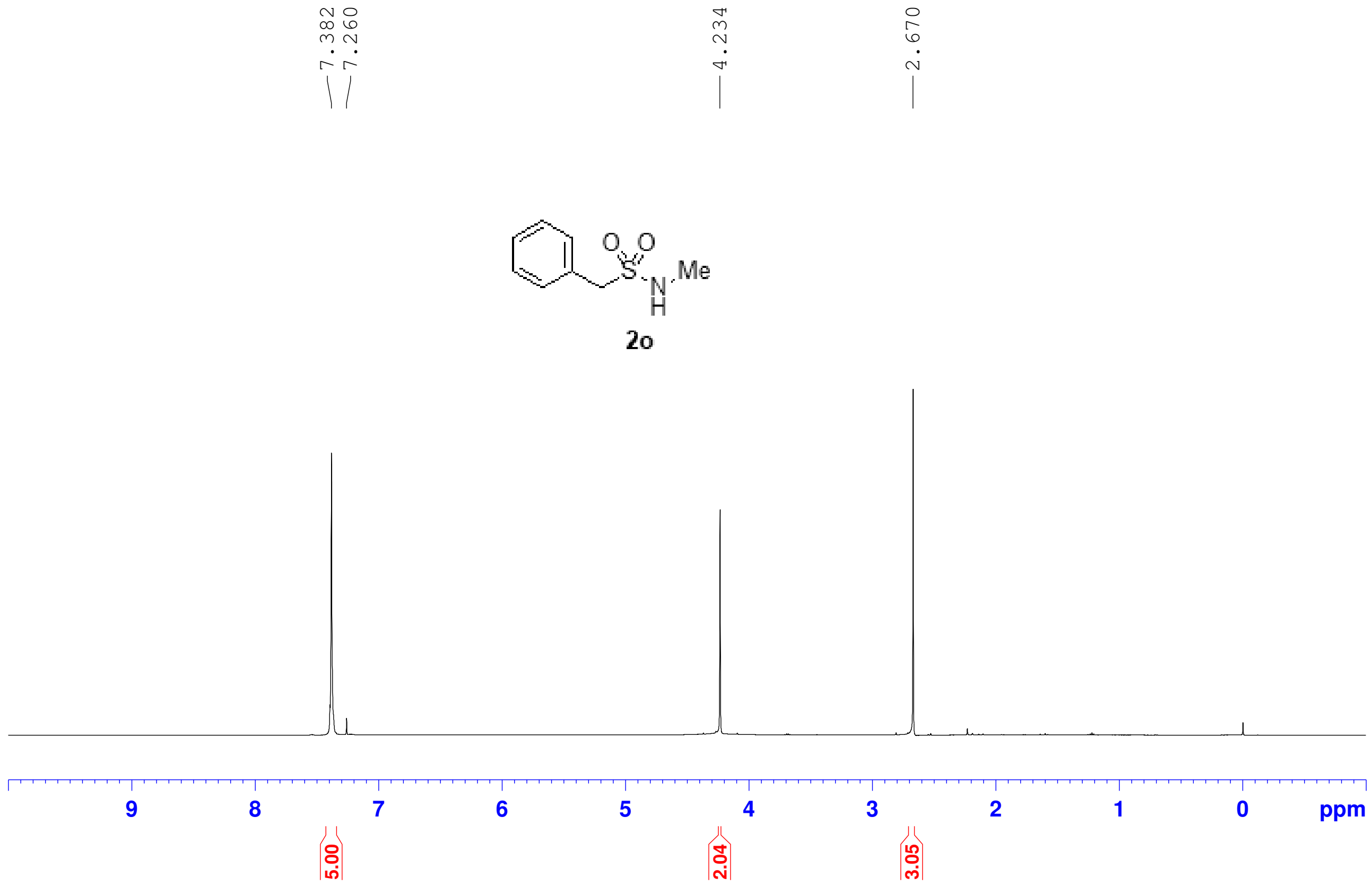
$\mathrm{N}$-methyl (phenyl) methanesulfonamide C13CPD CDCl3
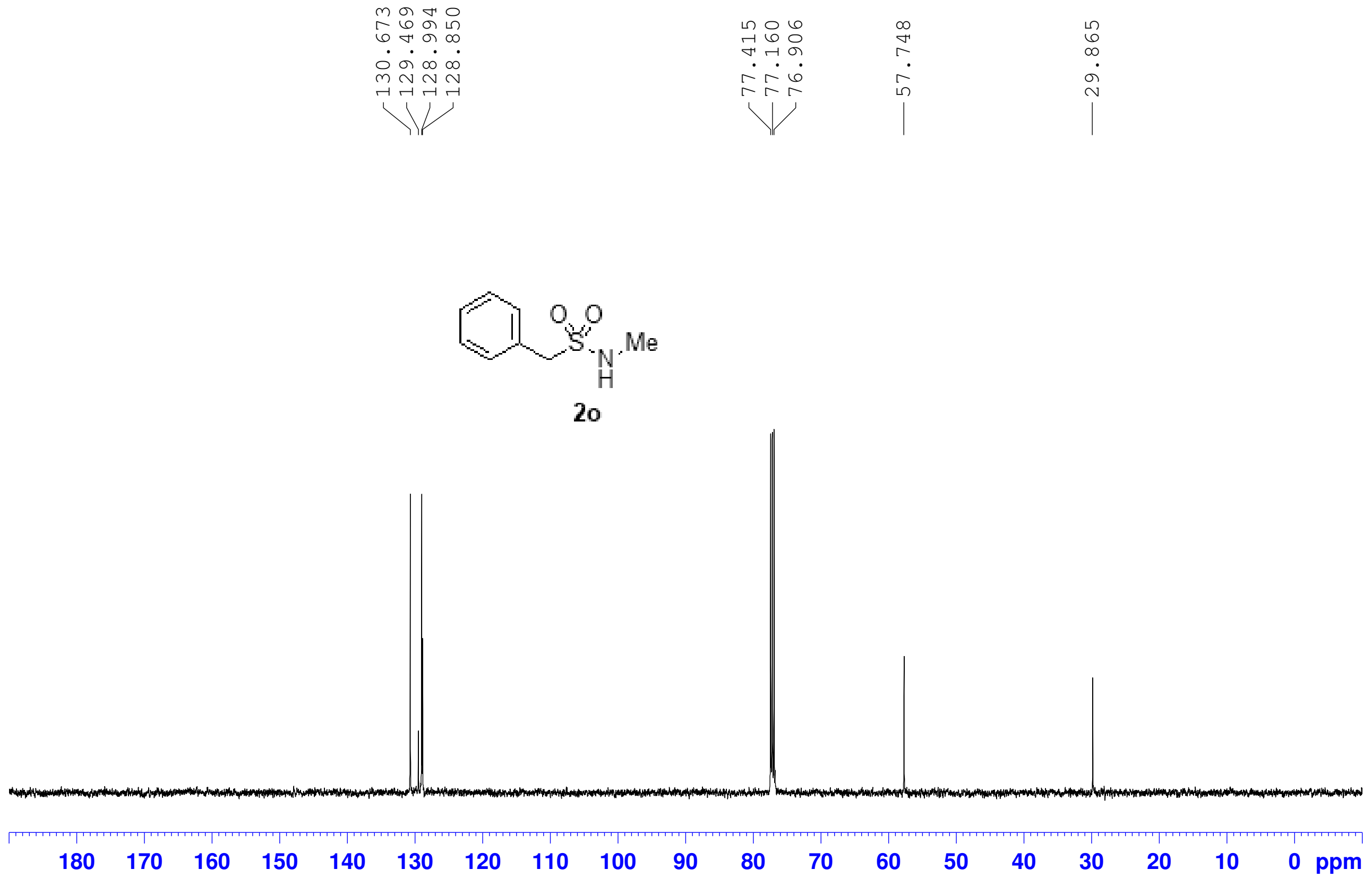
N, 2-dimethylpropane-2-sulfonamide Proton CDCl3

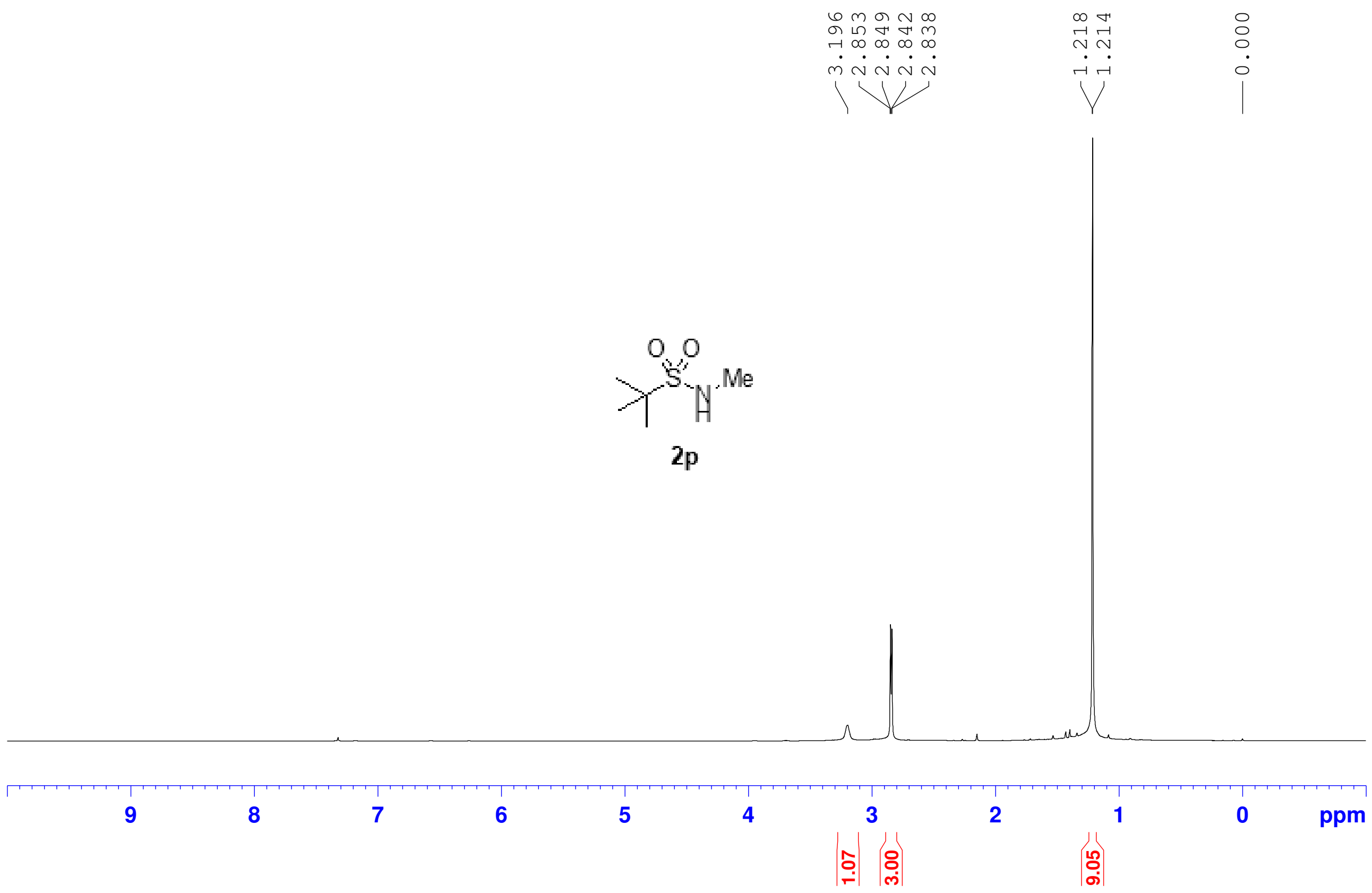


N, 2-dimethylpropane-2-sulfonamide C13CPD CDCl3
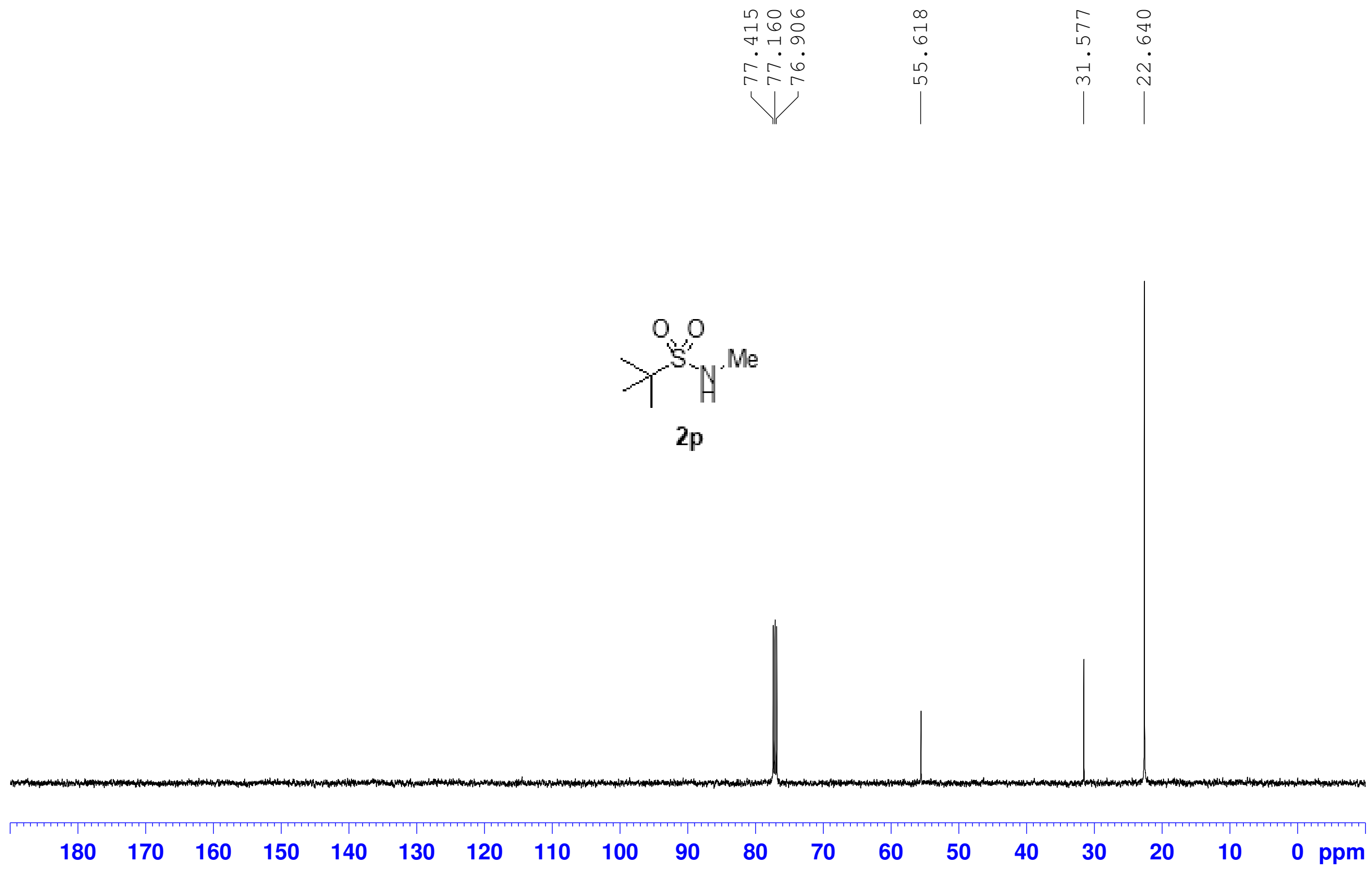
$\mathrm{N}$-methylcyclopropanesulfonamide Proton $\mathrm{CDCl} 3$

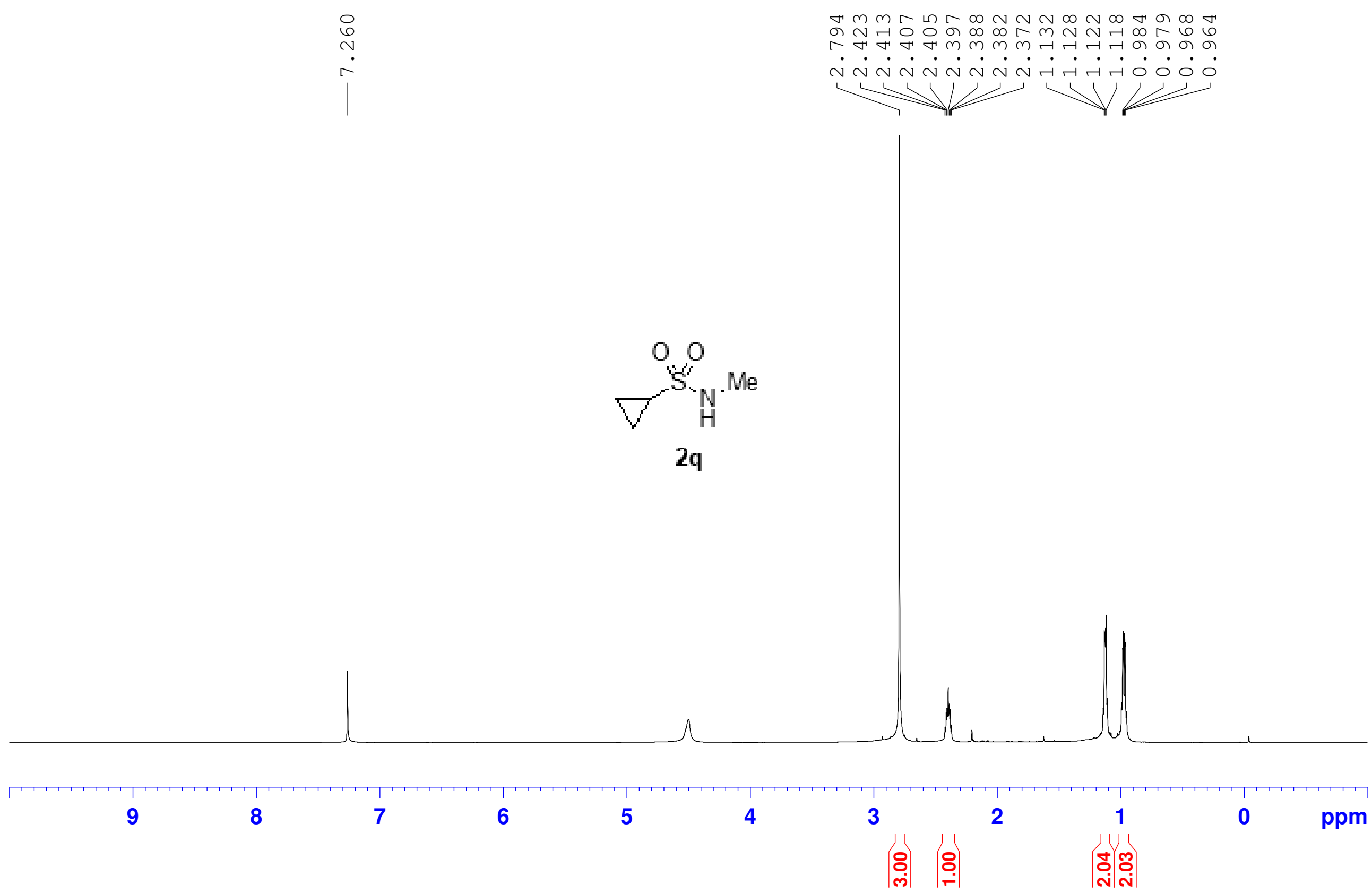



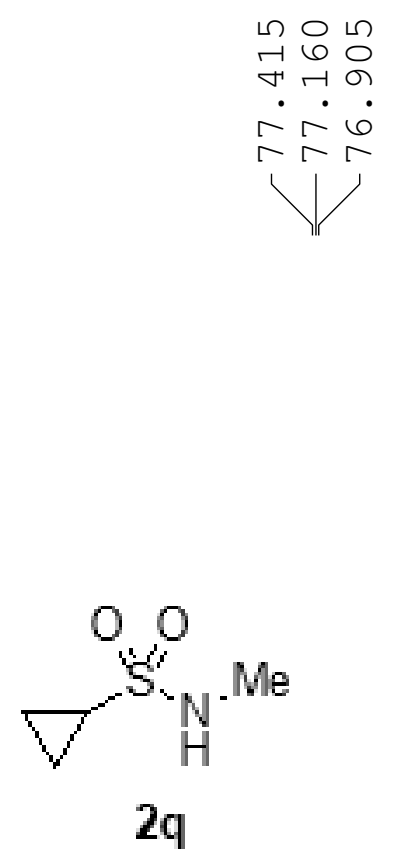

$\stackrel{m}{m} \stackrel{2}{n}$

เก เก

$\stackrel{\circ}{\sim} \stackrel{0}{\sim}$ 
$\mathrm{N}$-methylmethanesulfonamide Proton $\mathrm{CDCl} 3$

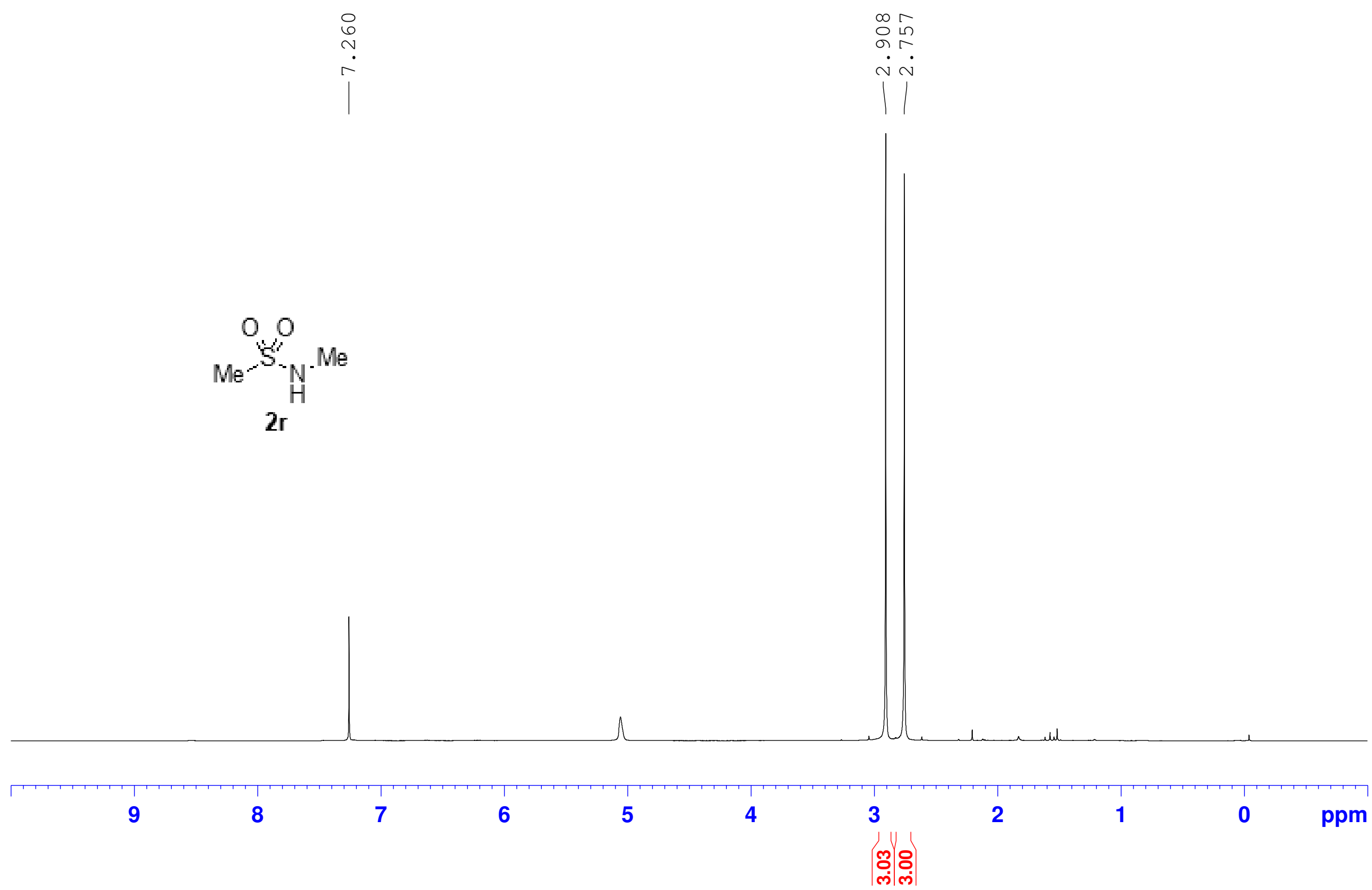


$\mathrm{N}$-methylmethanesulfonamide

C13CPD CDCl3
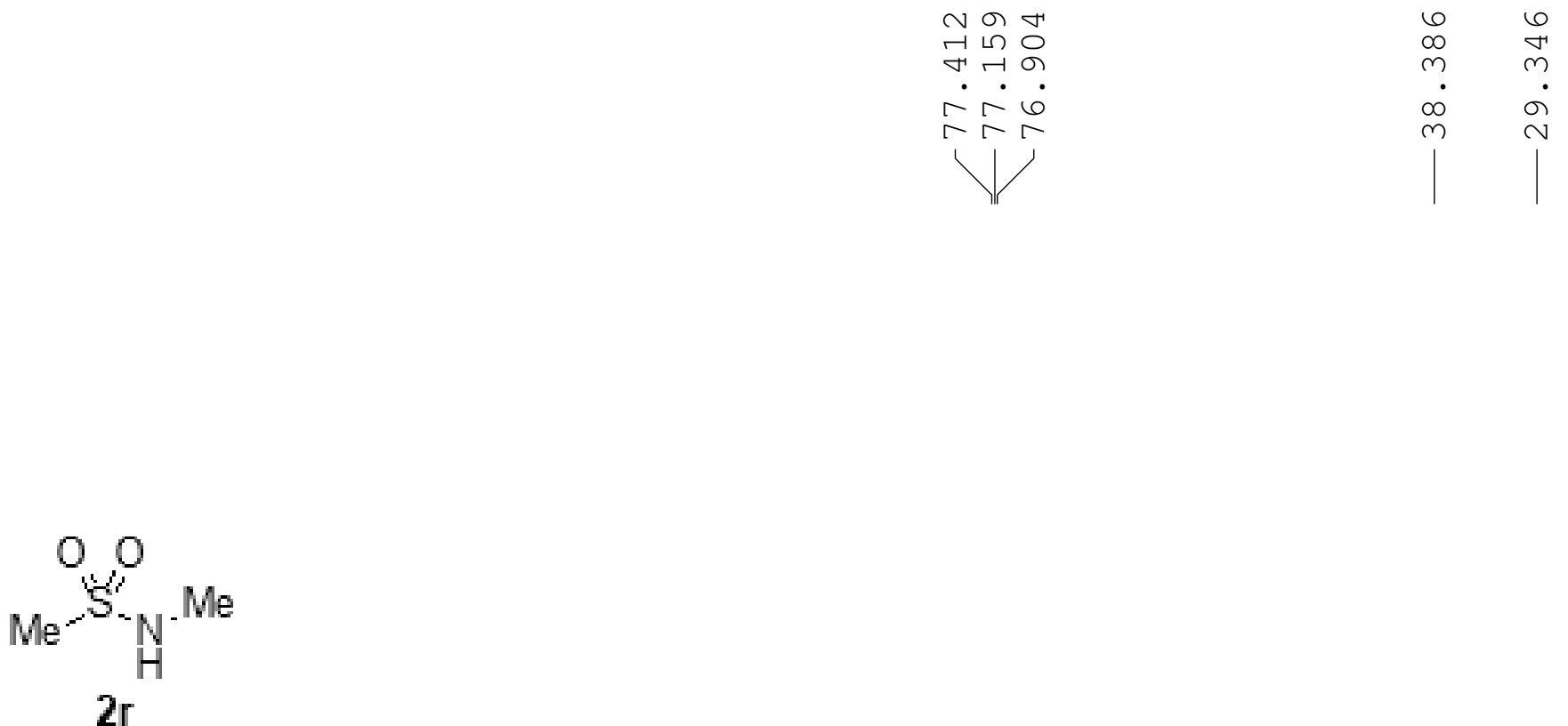

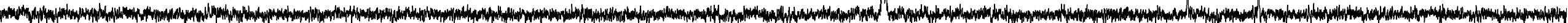


$\mathrm{N}$-methylbenzenamine

Proton CDCl3

ㅇํำ

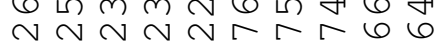

ㄷำ

$\longrightarrow 1$
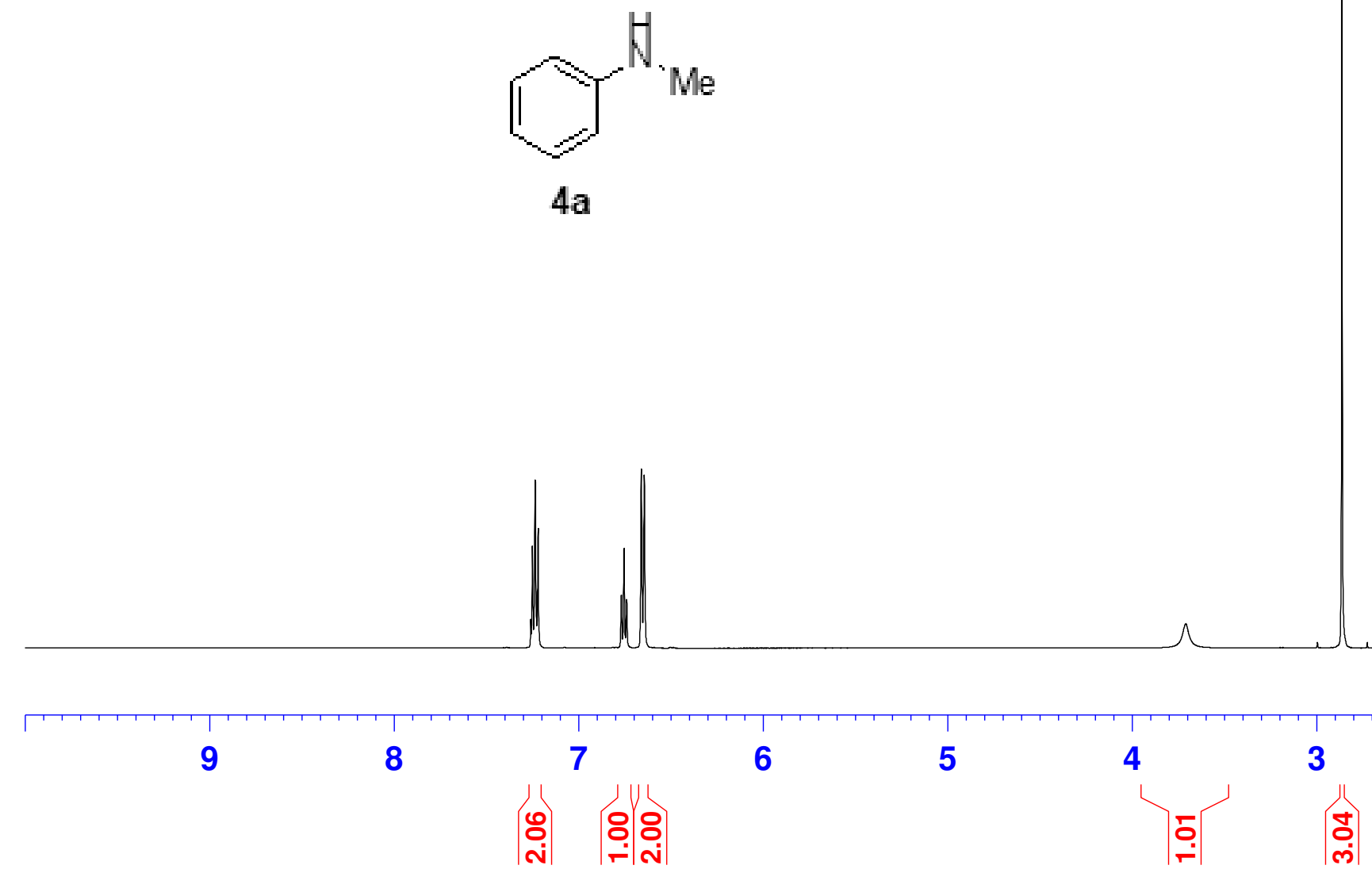


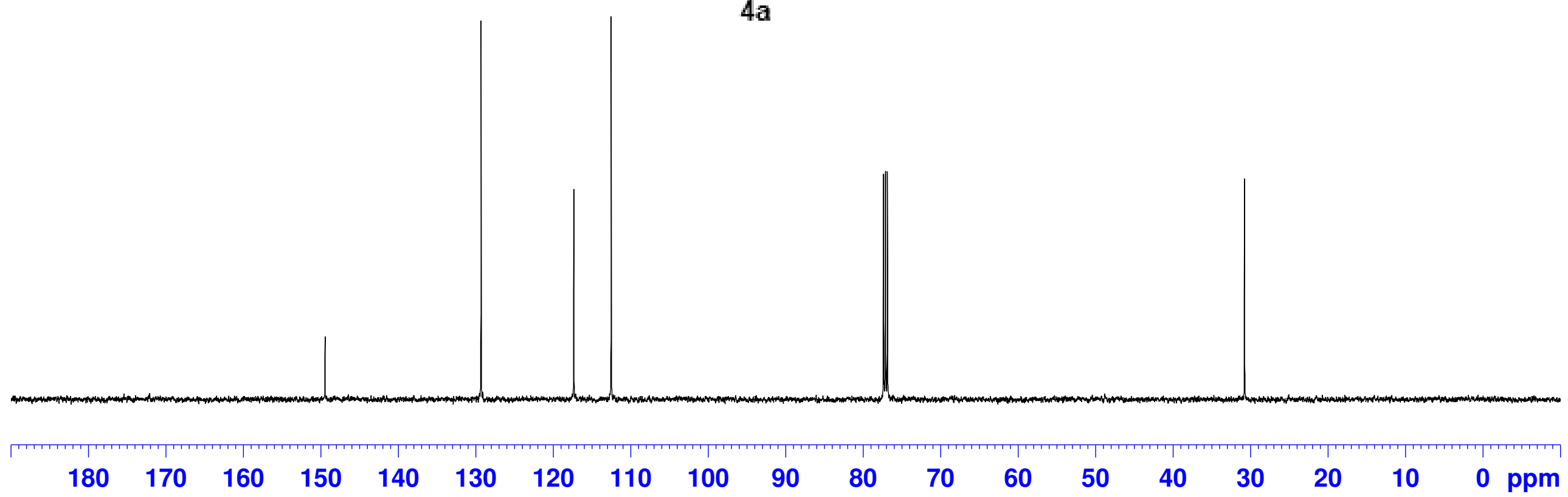


4-fluoro-N-methylbenzenamine Proton CDCl3

○

$m$ r

-

41<smiles>CNc1ccc(F)cc1</smiles>

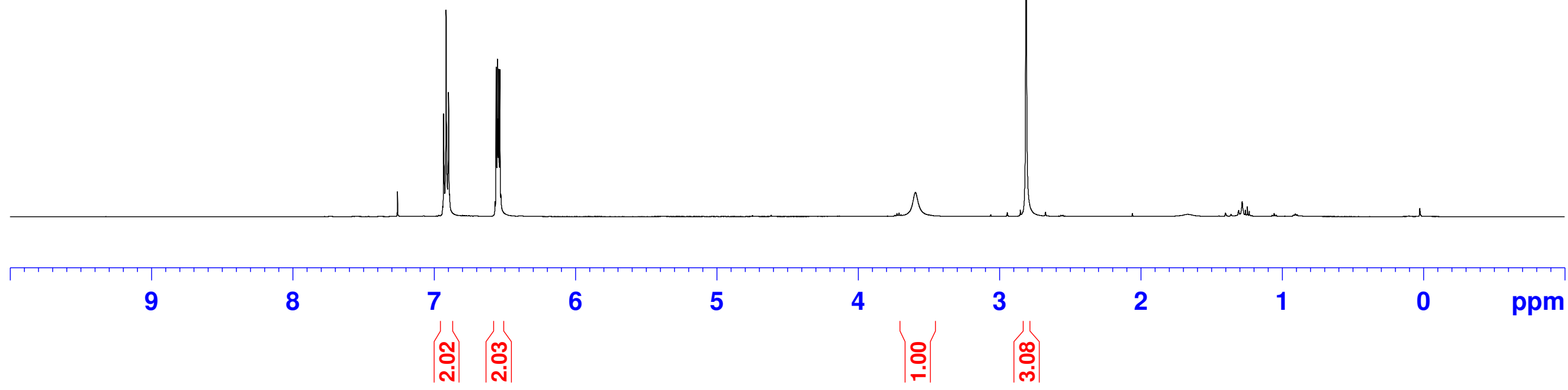



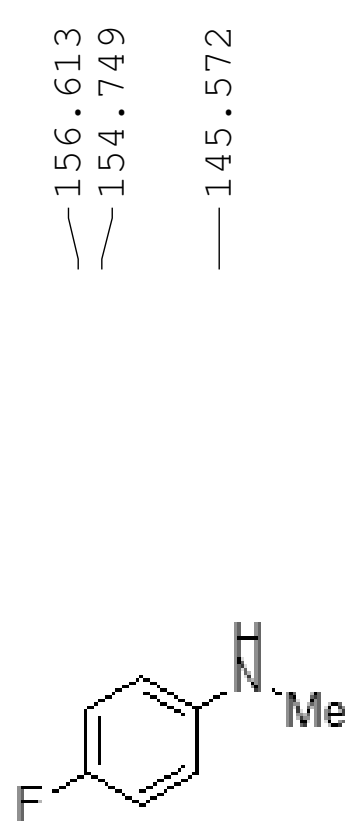

4b

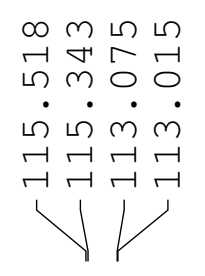

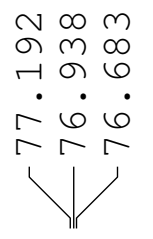

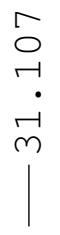

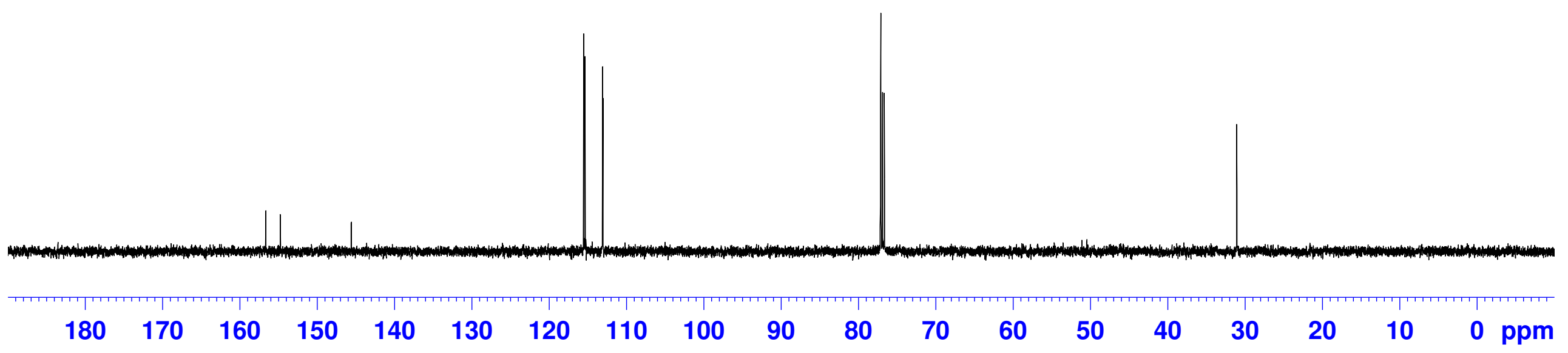



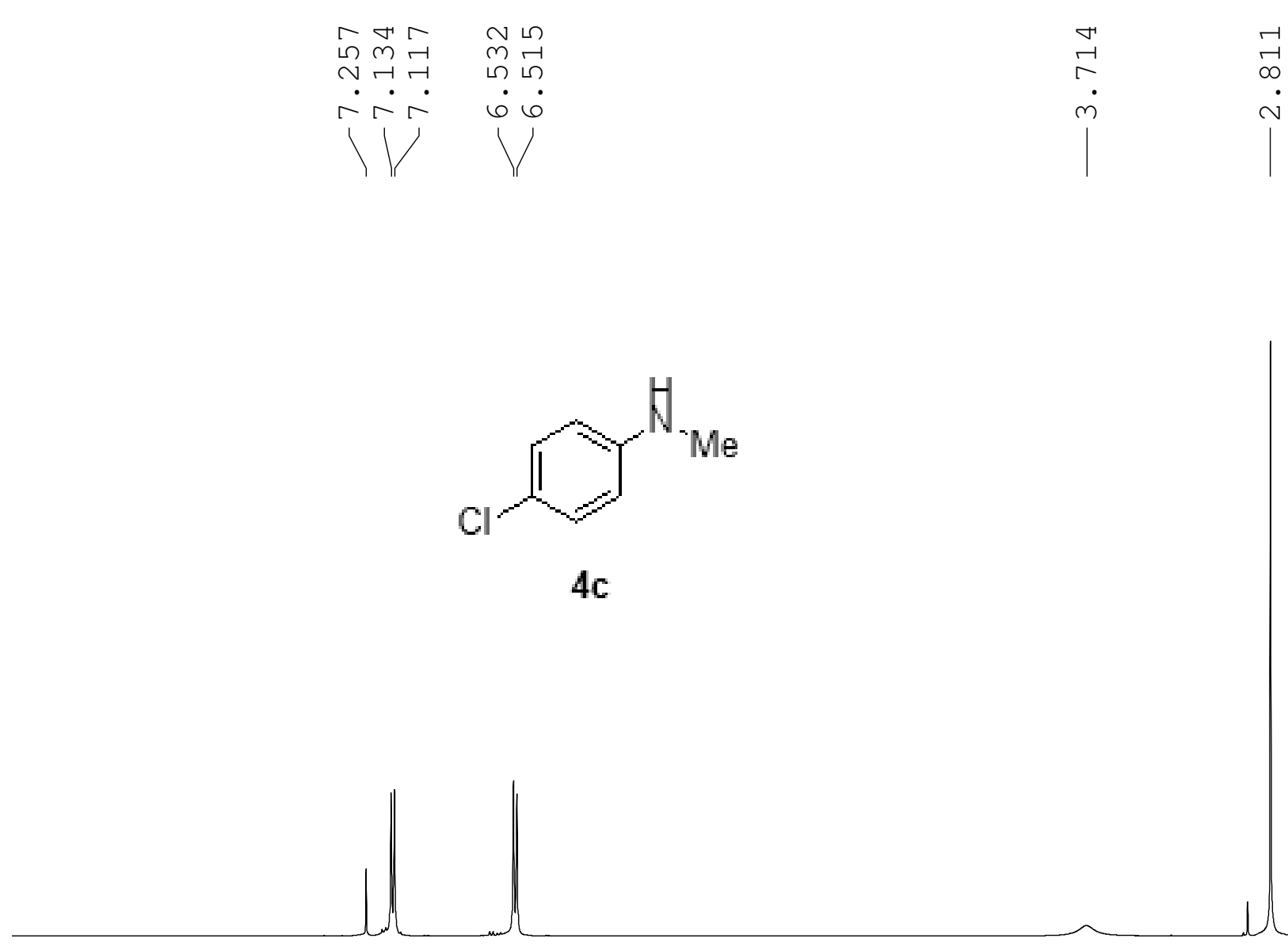

8.58 .0

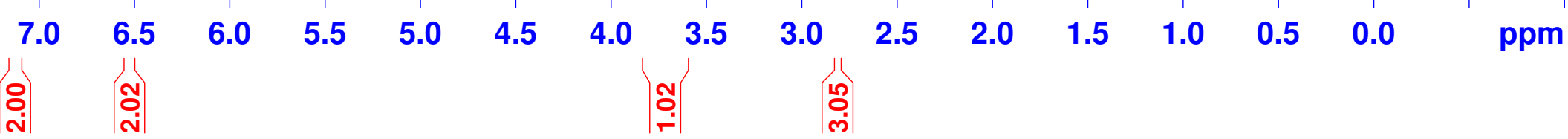


4-chloro-N-methylbenzenamine C13CPD CDCl3
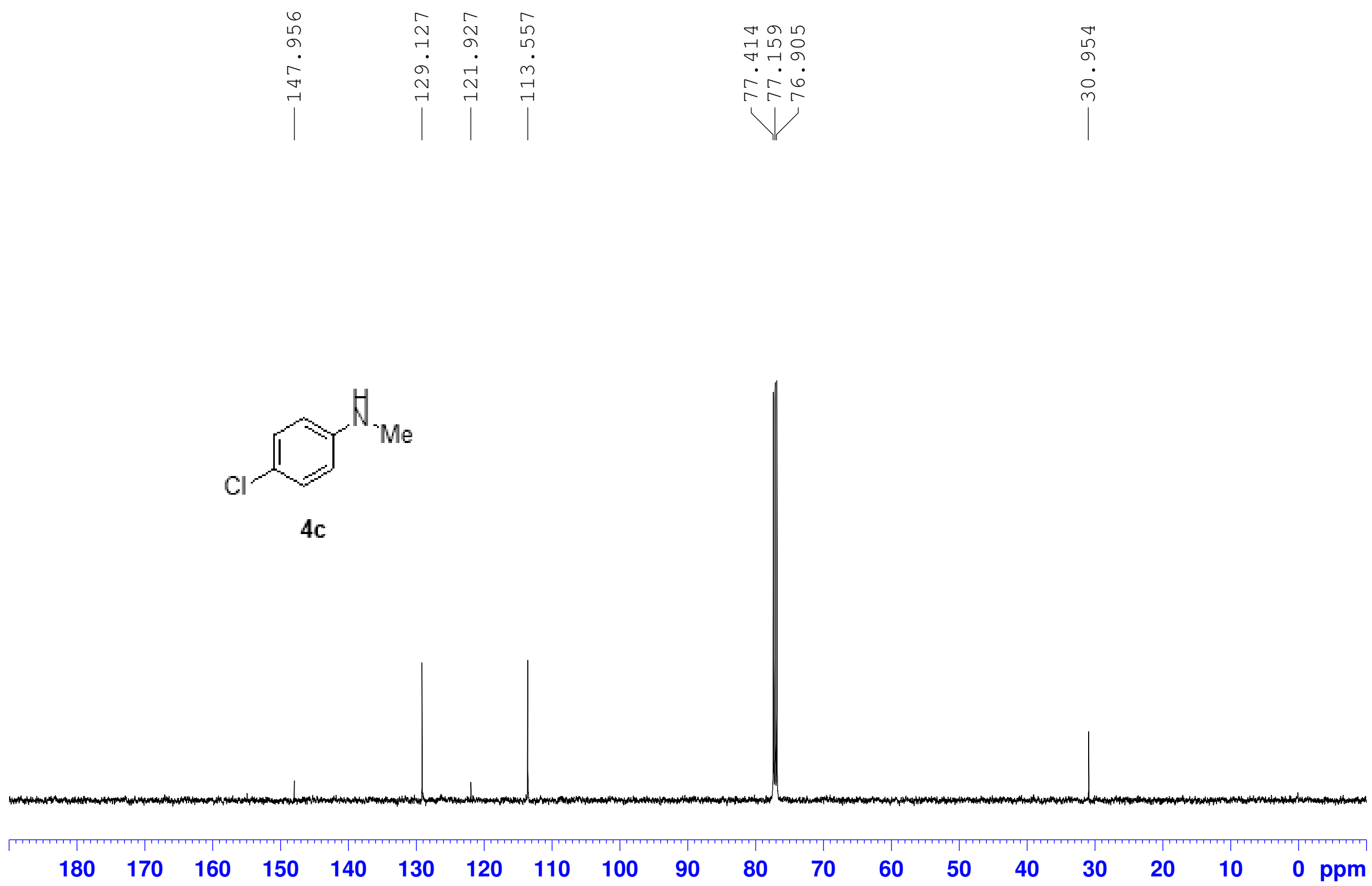
4-bromo-N-methylbenzenamine Proton $\mathrm{CDCl} 3$

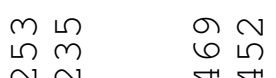

$$
\begin{aligned}
& \text { ก. } \\
& \therefore \dot{r} \dot{6} \\
& \text { V V }
\end{aligned}
$$
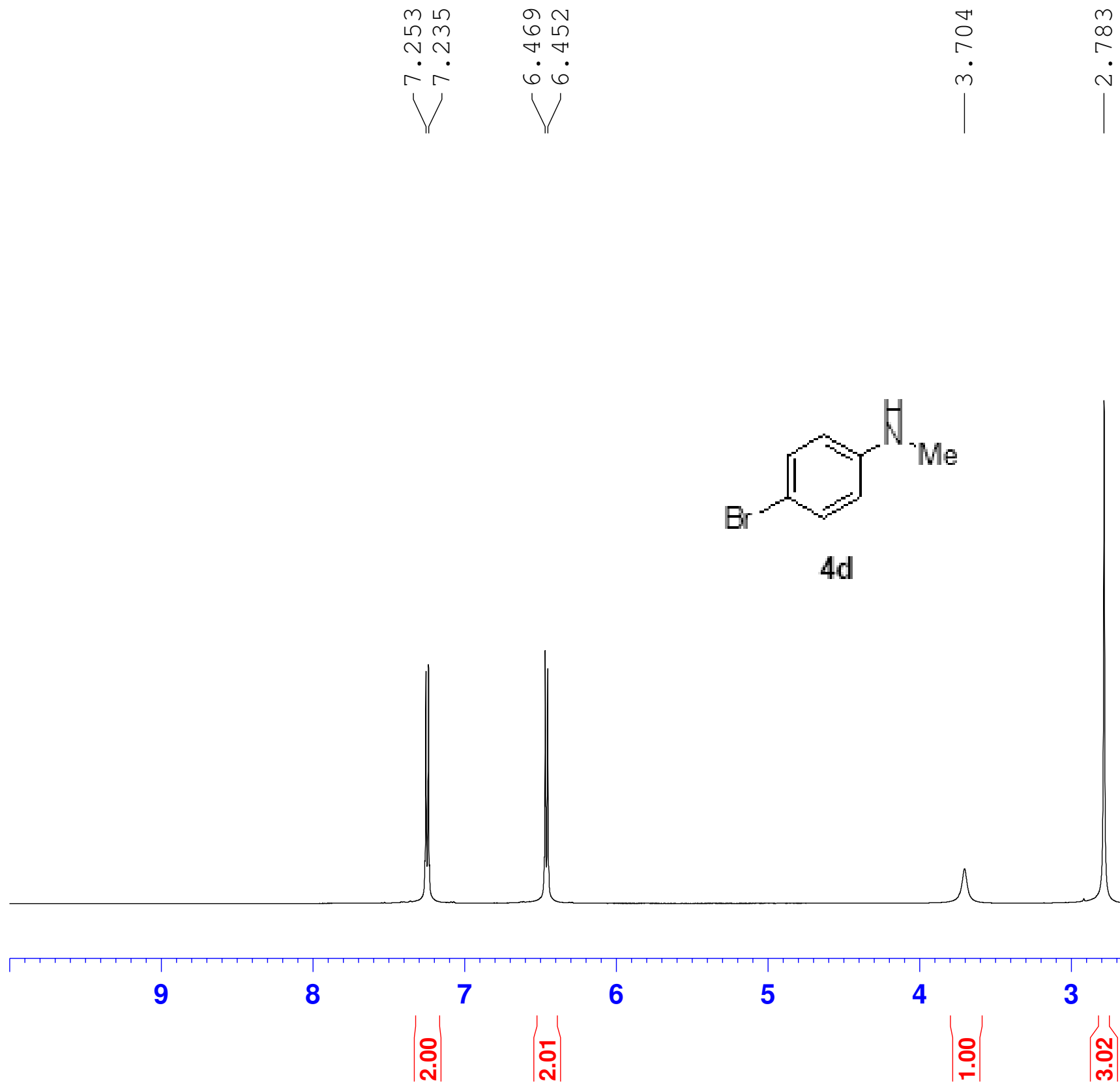

6

5

4

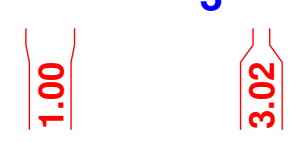



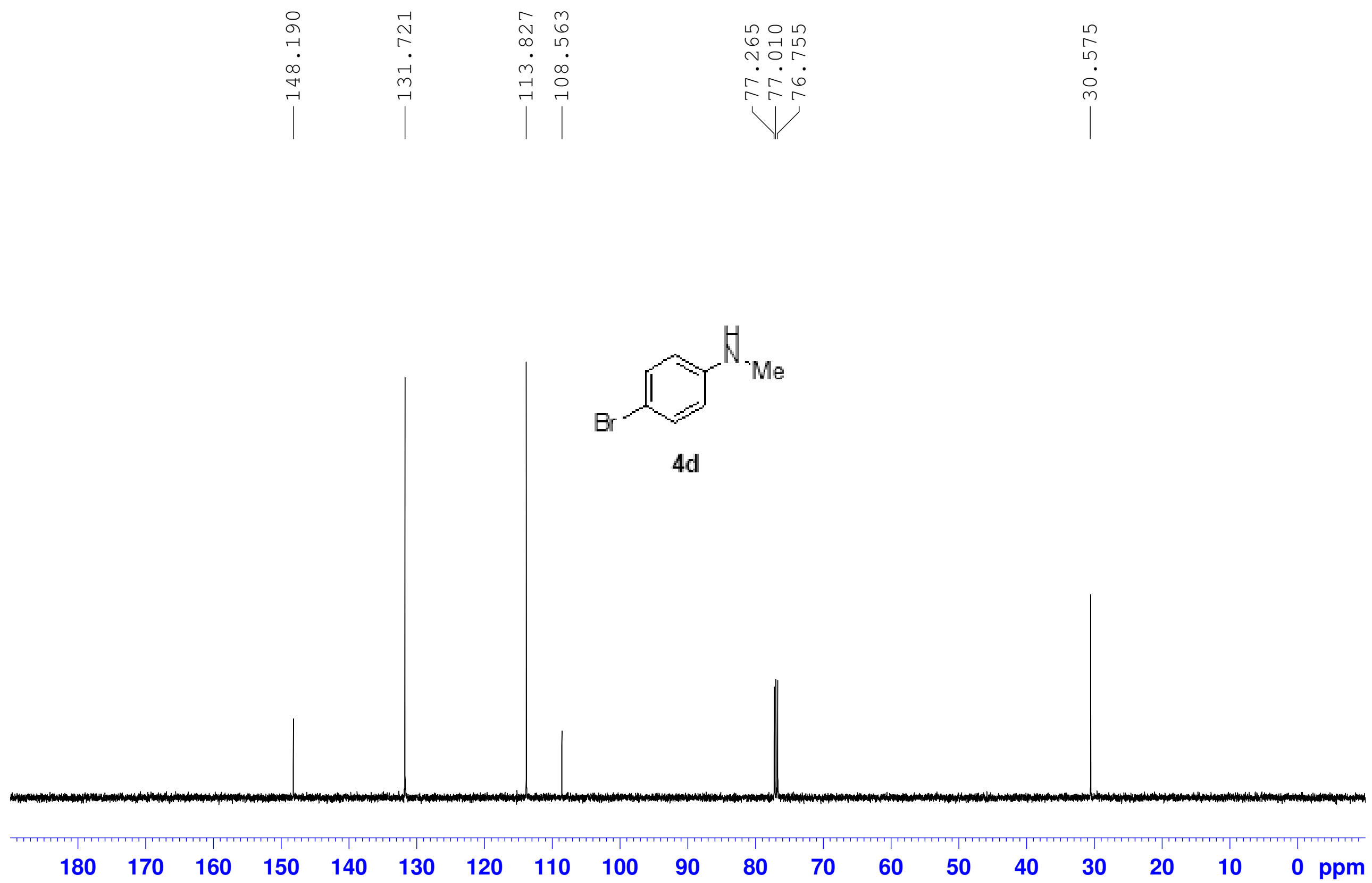

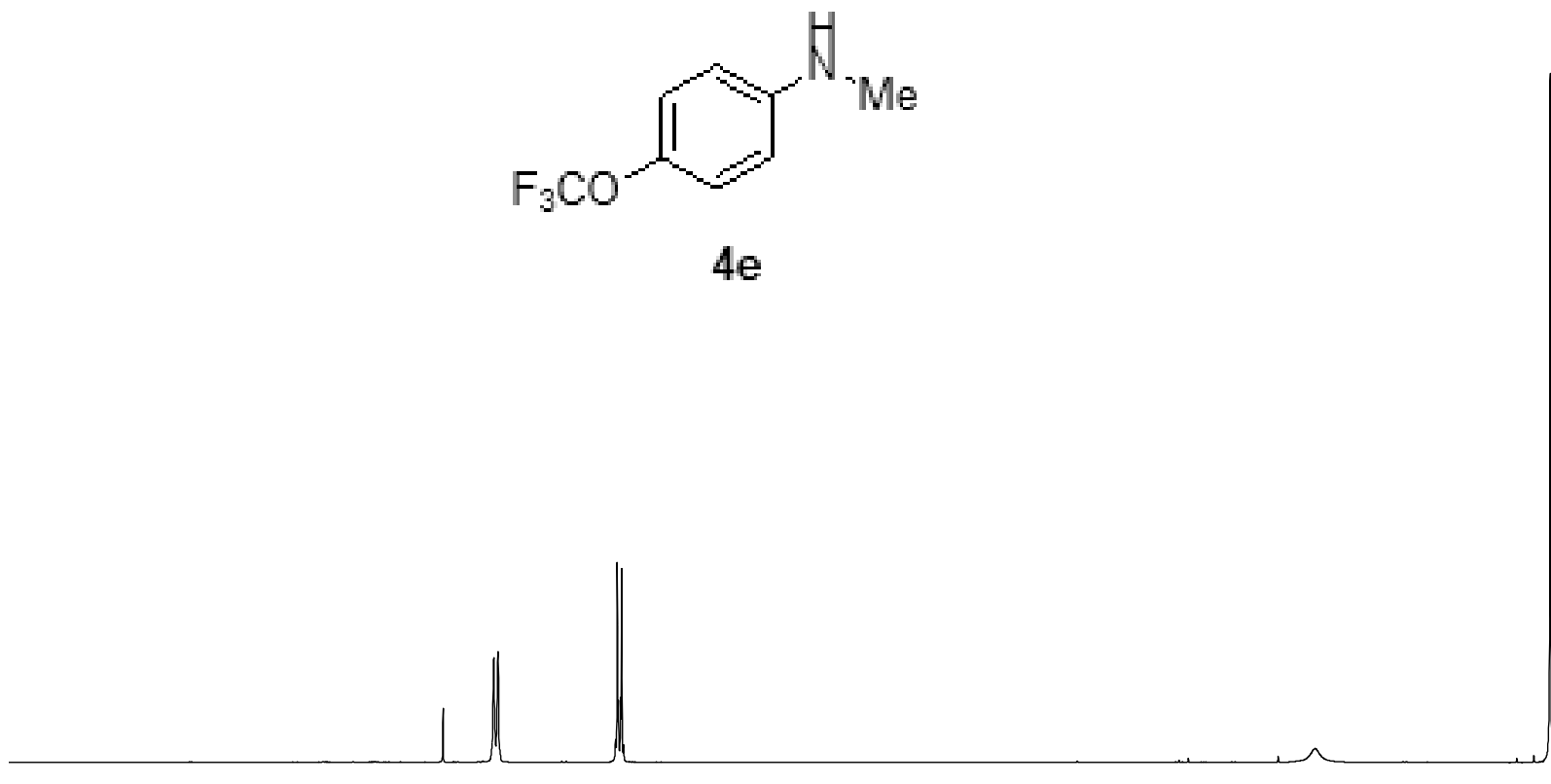

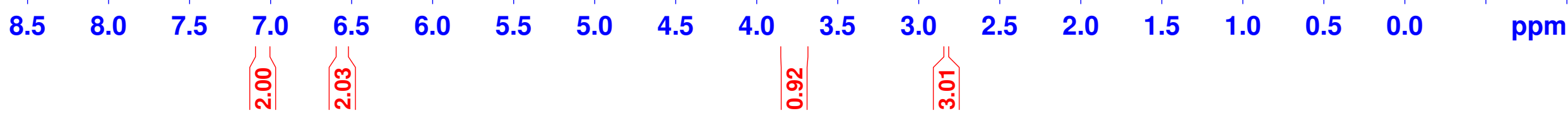


N-methyl-4-(trifluoromethoxy) benzenamine C13CPD CDCl3
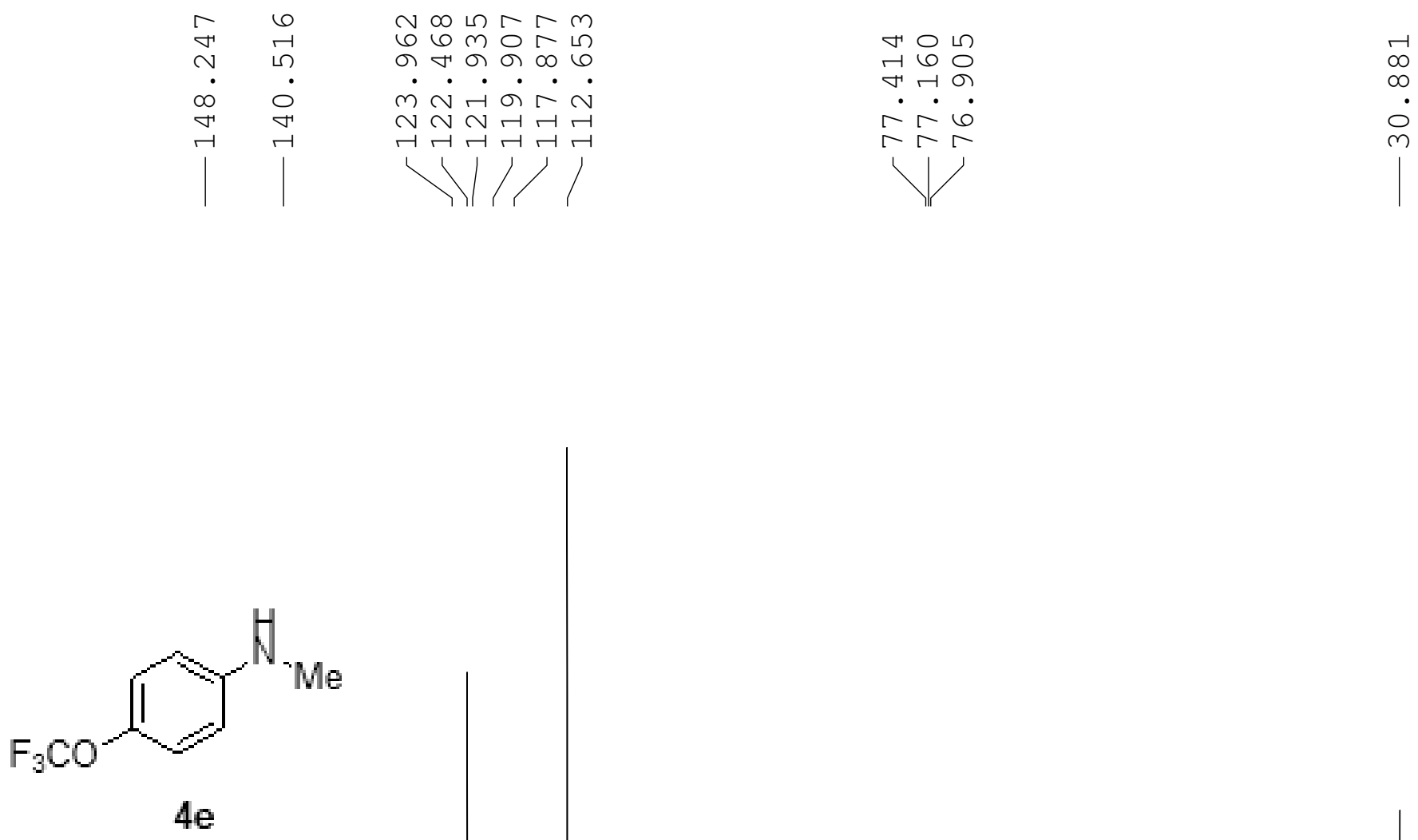
$\mathrm{N}, 4$-dimethylbenzenamine

Proton $\mathrm{CDCl} 3$

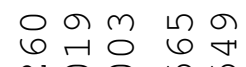

$$
\begin{aligned}
& \text { vo } 0 \text { เ เ } \\
& \therefore \sim \dot{ } \\
& 1 V
\end{aligned}
$$
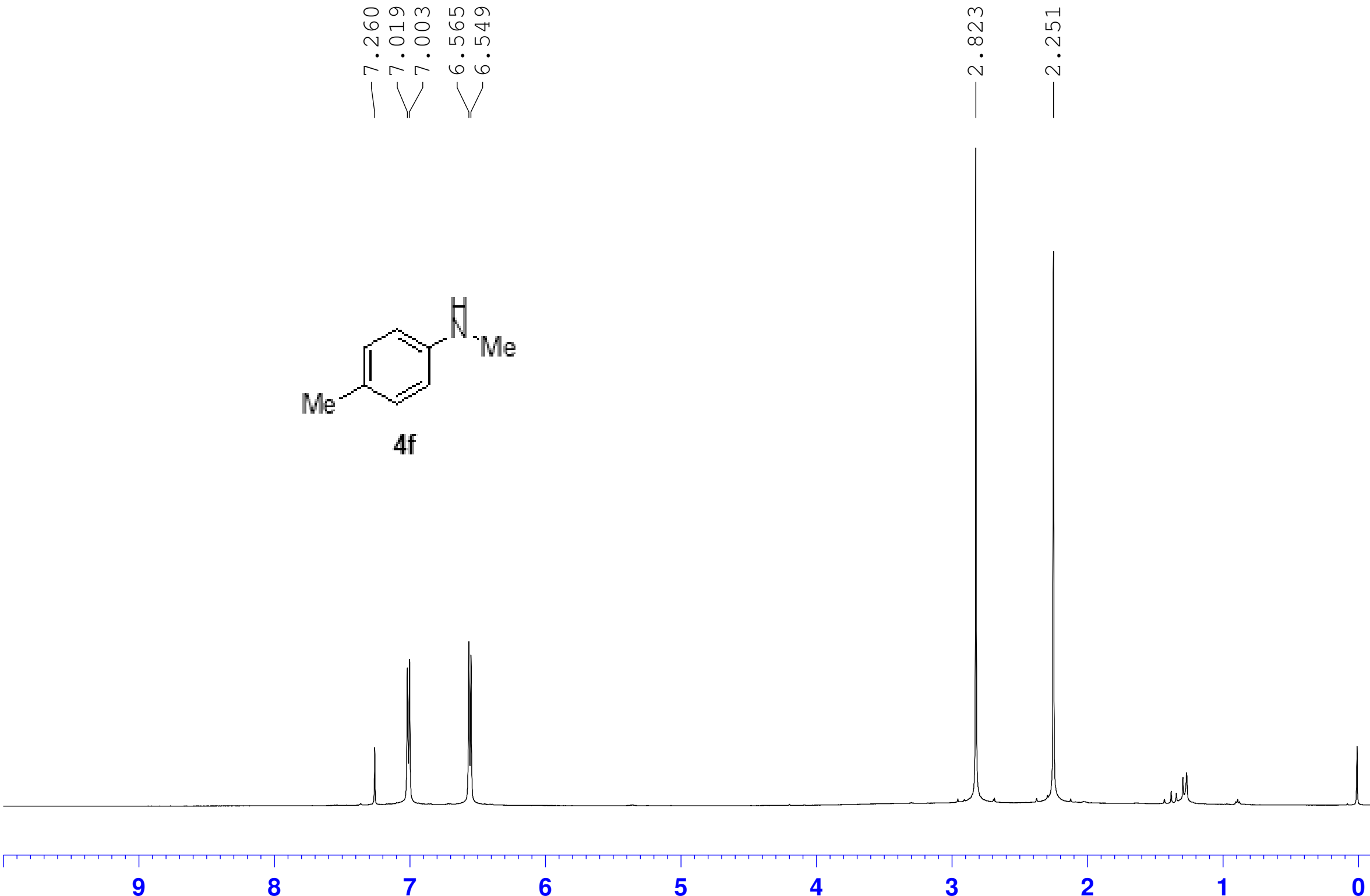

8

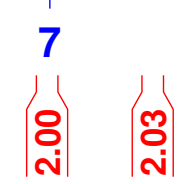

6

5

4

3

|人 


\begin{tabular}{|c|c|c|c|c|c|}
\hline 0 & $\nabla \sigma$ & 6 & & & \\
\hline$m$ & $r \in$ & 0 & $N r m$ & 6 & $\infty$ \\
\hline 0 & in $m$ & ம & $\sigma m \infty$ & r & ம \\
\hline - & & $\therefore$ & † の 6 & $\sigma$ & $\sim$ \\
\hline - & ( 6 & $\sim$ & $\dot{0} \dot{0}$ & $\dot{\sim}$ & \\
\hline $\begin{array}{r}\vec{r} \\
\overrightarrow{1}\end{array}$ & $\sim N$ & ت & $\begin{aligned}- & 0 \\
1 & \end{aligned}$ & $\stackrel{\circ}{m}$ & $\stackrel{\bigcirc}{\sim}$ \\
\hline & & & & & \\
\hline
\end{tabular}
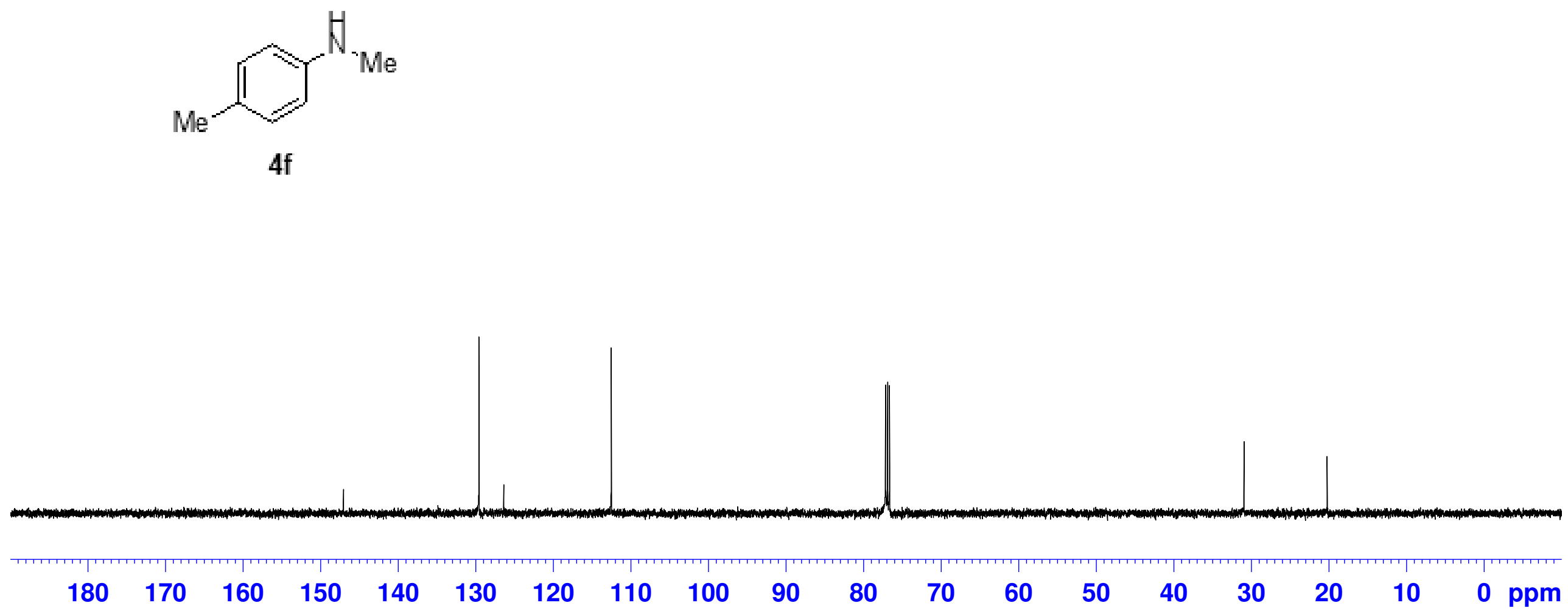
4-chloro-N, 3-dimethylbenzenamine Proton $\mathrm{CDCl} 3$

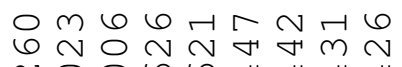

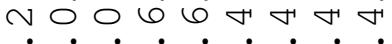

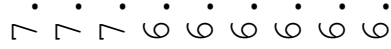

11
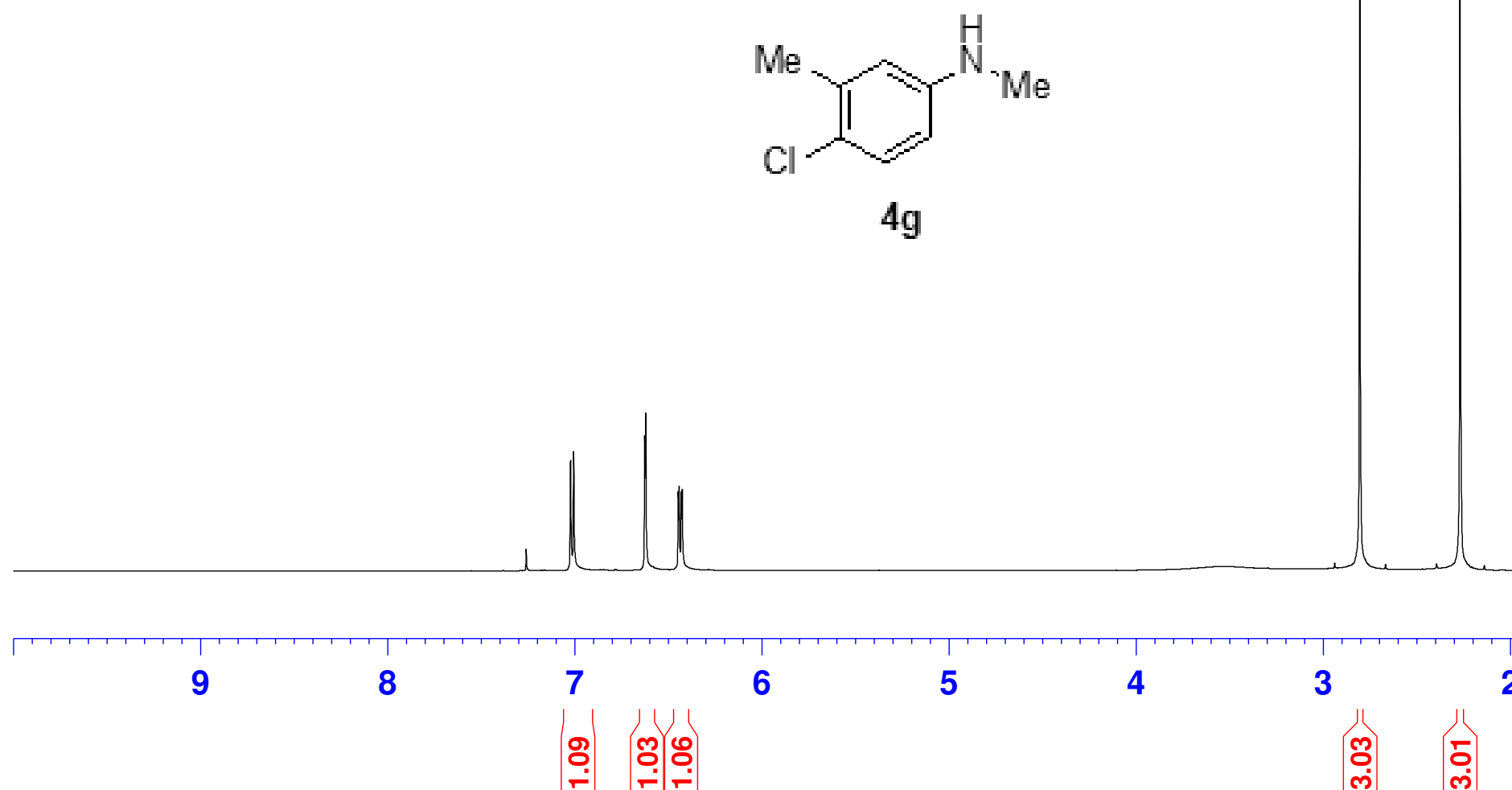
<smiles>CNc1ccc(Cl)c(C)c1</smiles>

$4 \mathrm{~g}$

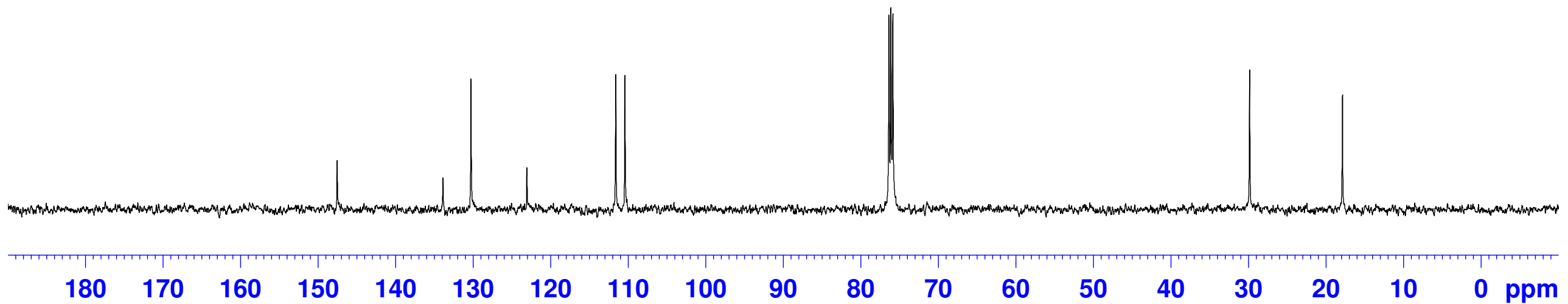


3-methoxy-N-methylbenzenamine

Pronton CDCl3
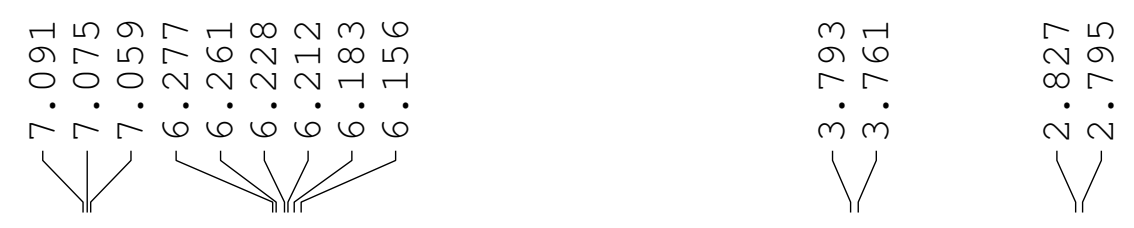

0
0
0
0
1
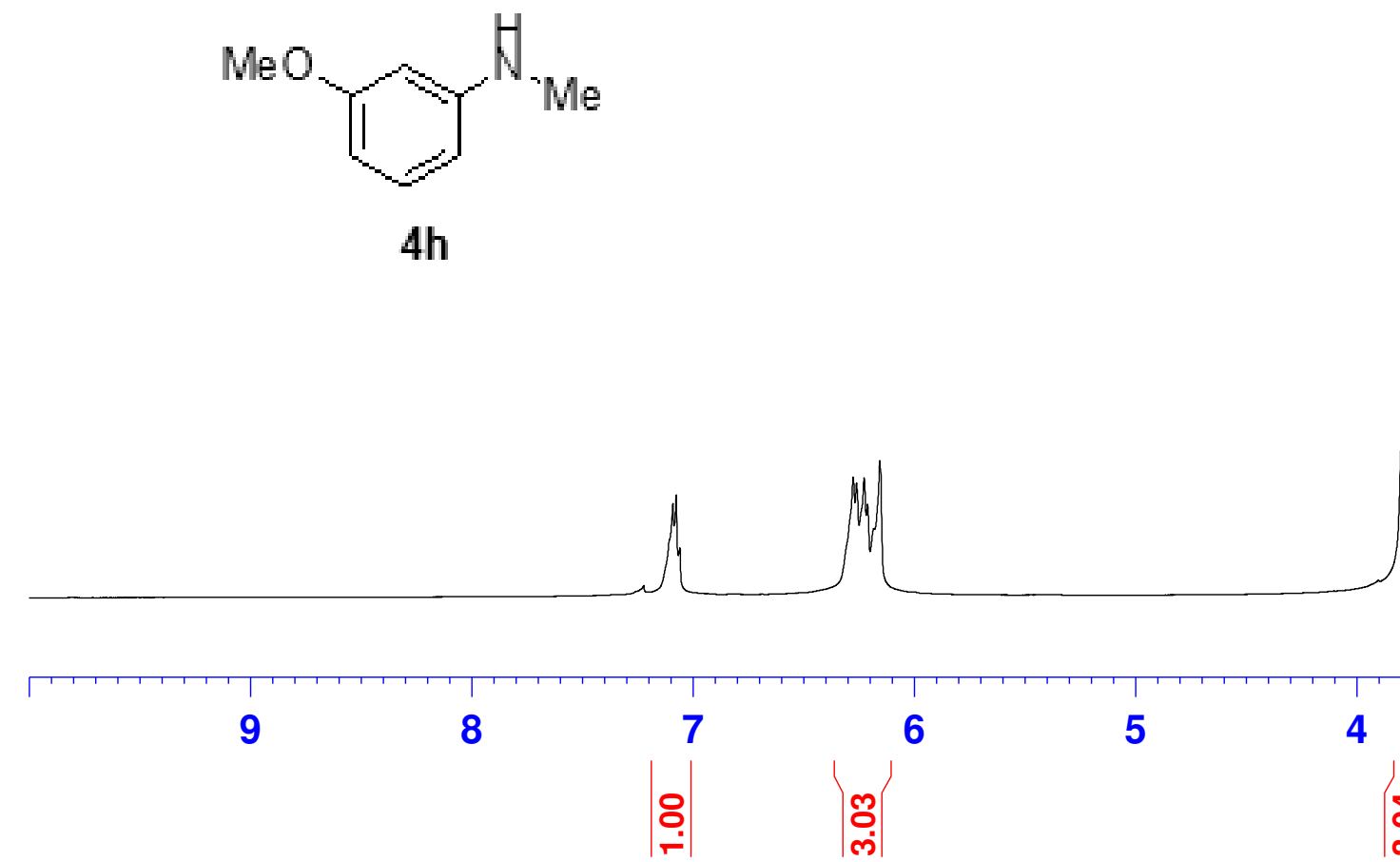

m.
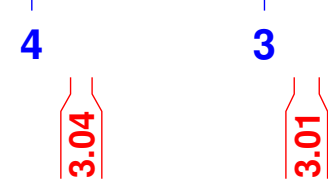

$\mid \begin{array}{ll}\text { 이 } \\ \text { ले }\end{array}$


<smiles>CNc1cccc(OC)c1</smiles>

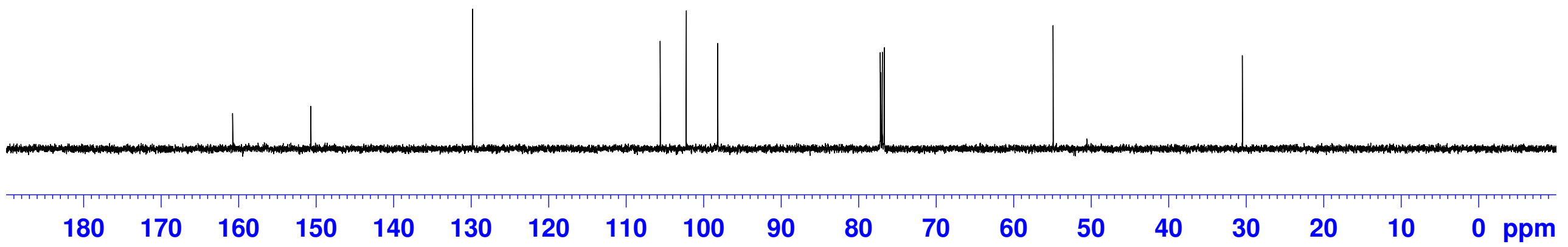


のत म०

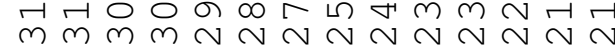

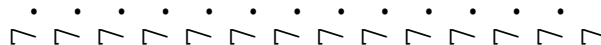

$\longrightarrow$
๙

Nin

$\dot{m} \dot{m} \dot{m}$
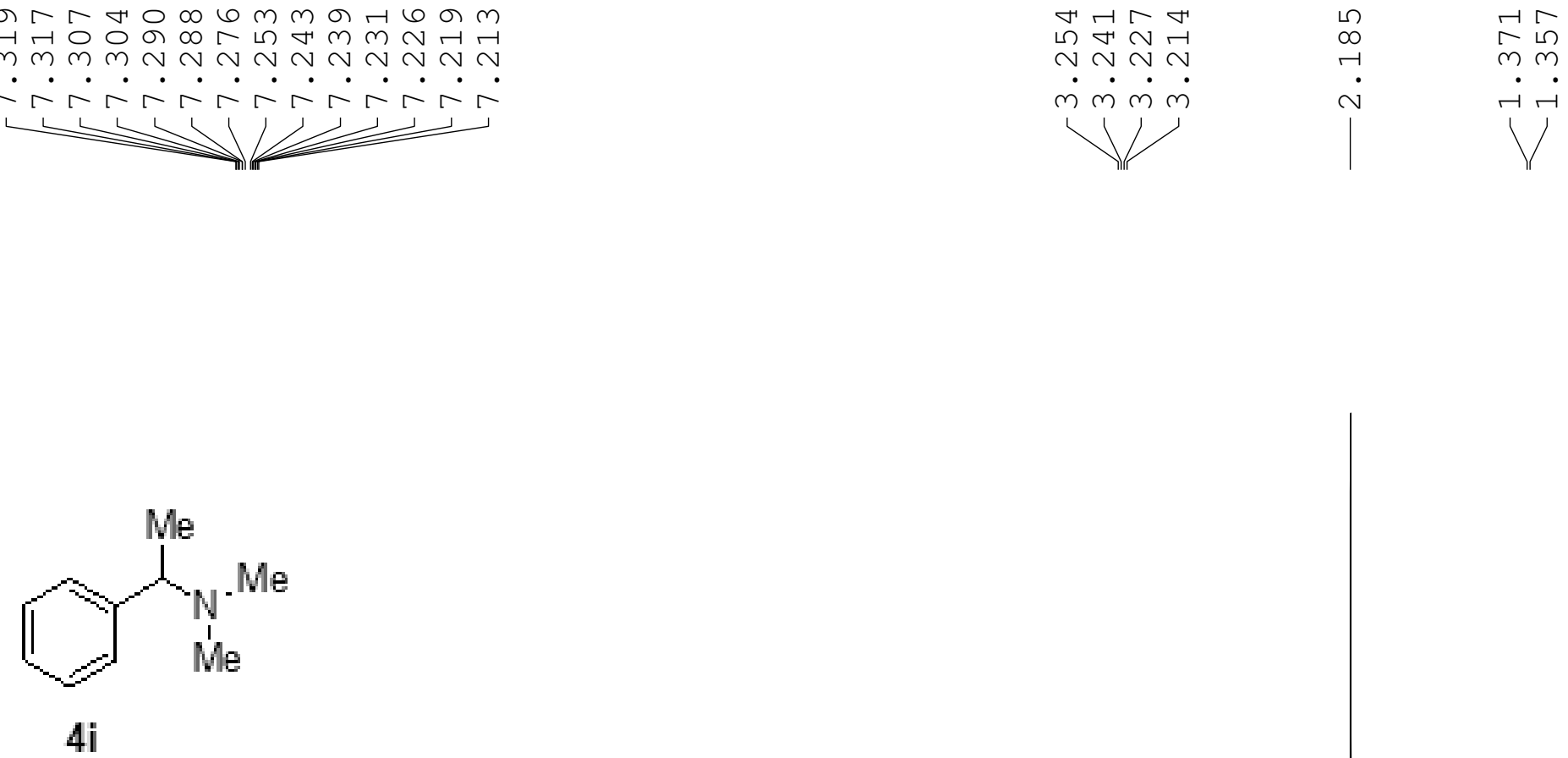

1

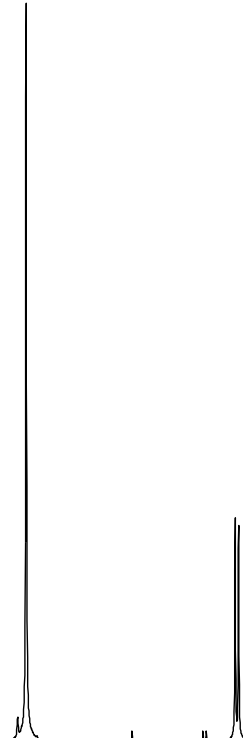

\begin{tabular}{|c|c|c|c|c|c|c|c|c|c|c|c|c|c|c|c|c|c|}
\hline 8.5 & 8.0 & 7.5 & 7.0 & 6.5 & 6.0 & 5.5 & 5.0 & 4.5 & 4.0 & 3.5 & 3.0 & 2.5 & 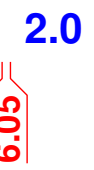 & $\begin{array}{l}1.5 \\
0 \\
\text { ल }\end{array}$ & 1.0 & 0.5 & 0.0 \\
\hline
\end{tabular}



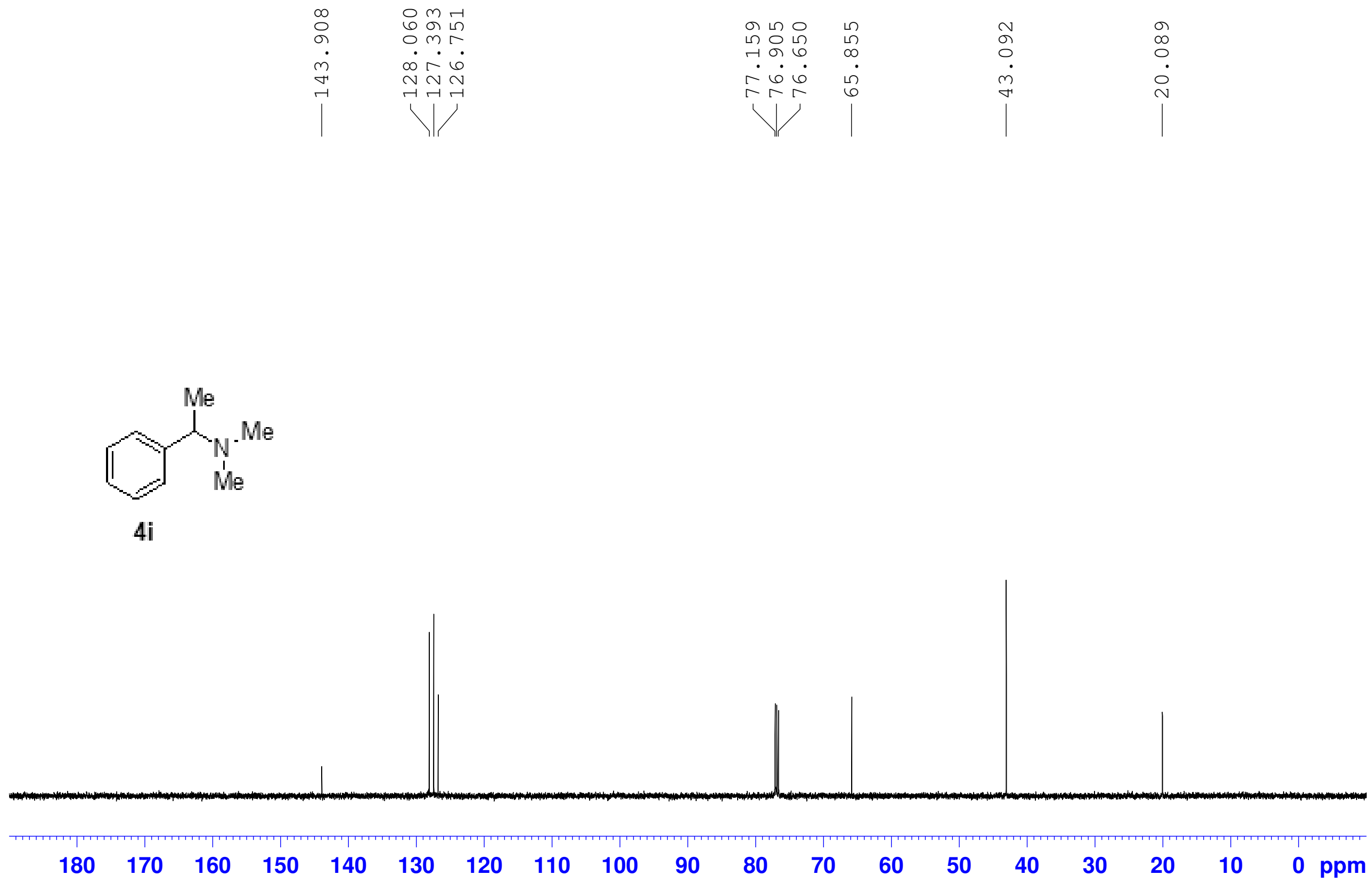
1-N-methyl-adamantylamin

Proton $\mathrm{CDCl} 3$

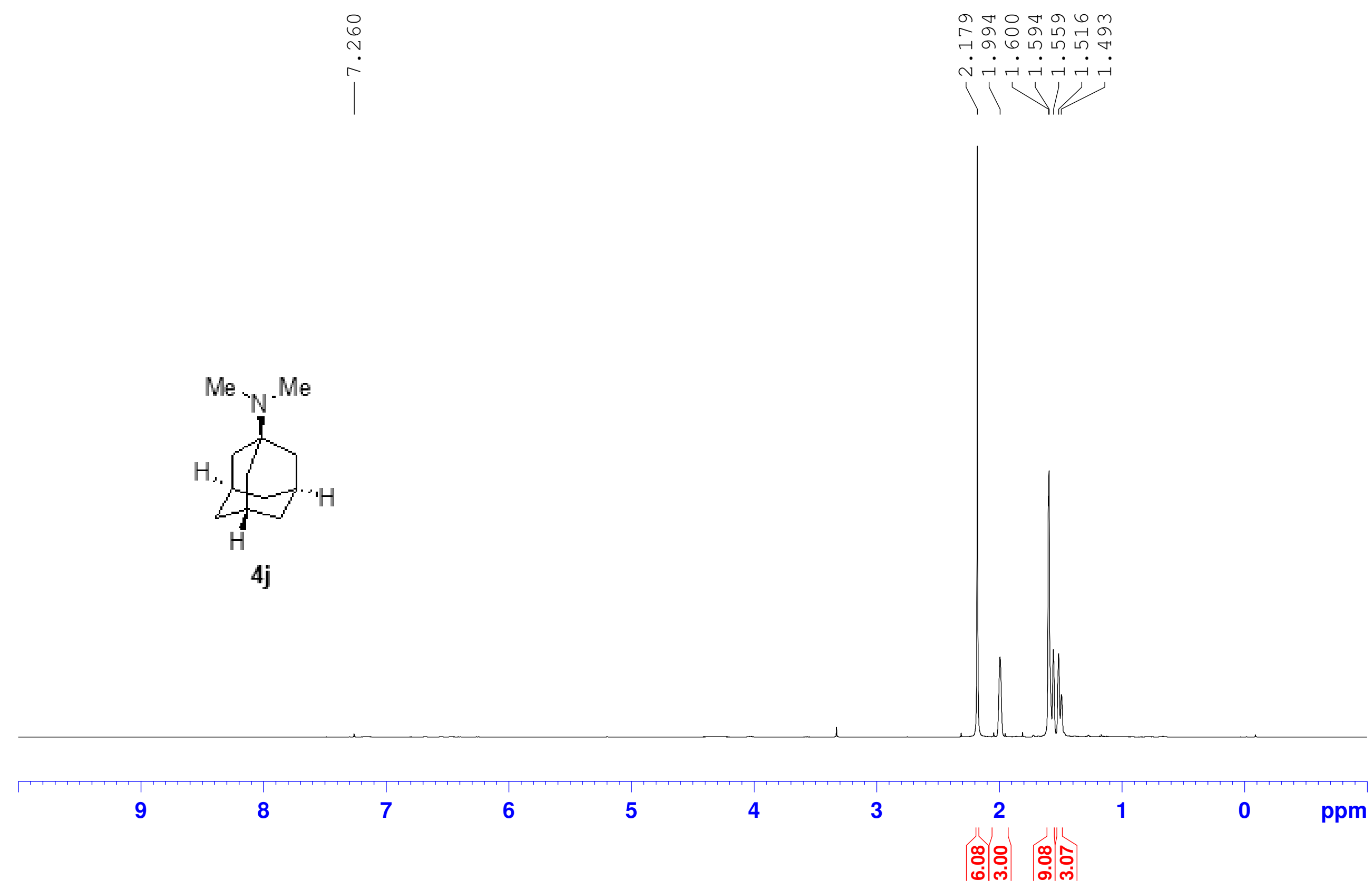


1-N-methyl-adamantylamin

C13CPD CDCl3
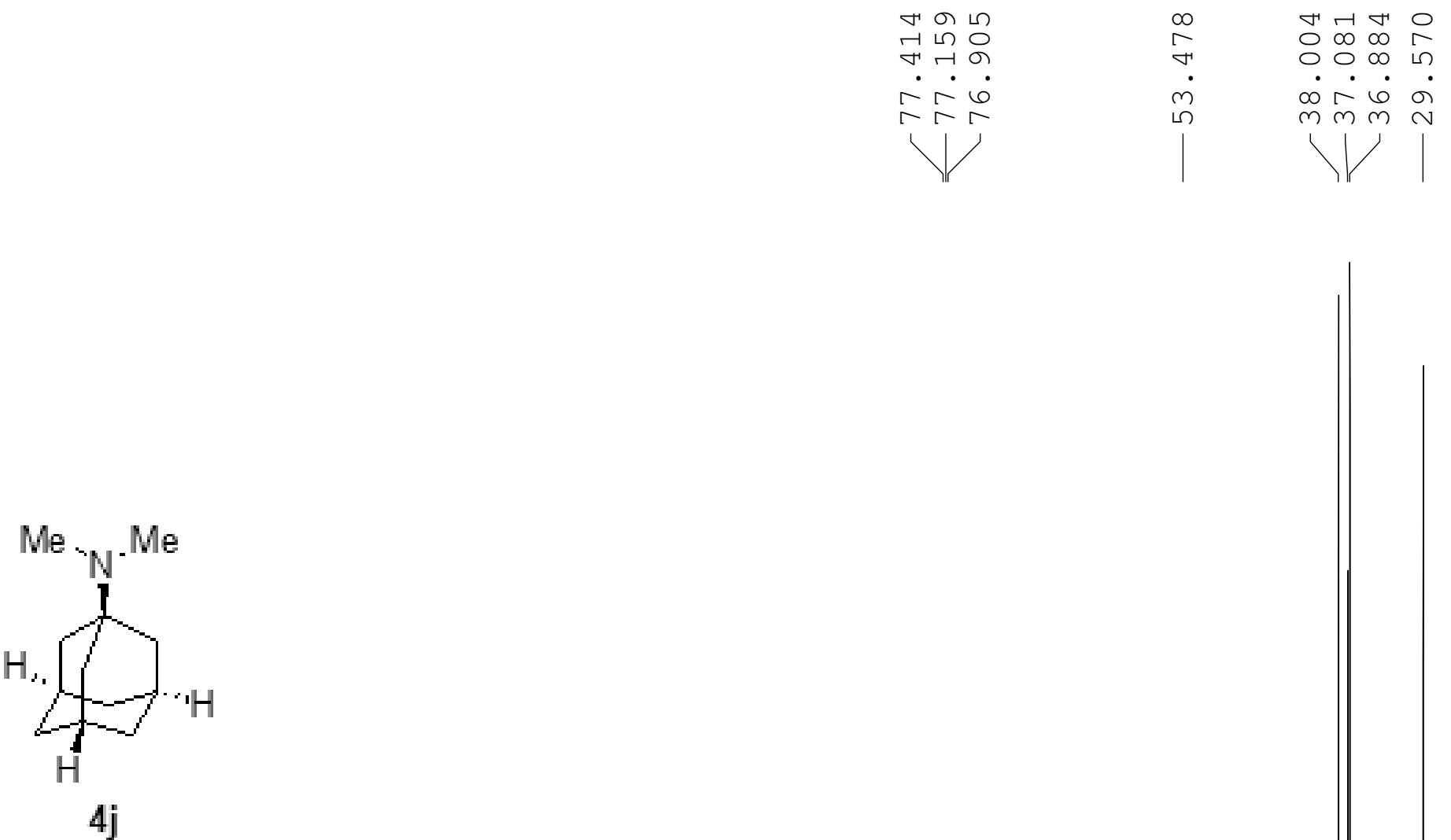
2-methyl-1,2,3,4-tetrahydroisoquinoline Proton $\mathrm{CDCl} 3$

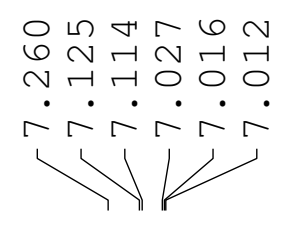

๓

เ न の

$\dot{m} \dot{\sim} \dot{\sim} \dot{\sim} \dot{\sim} \dot{\sim}$

$+1$
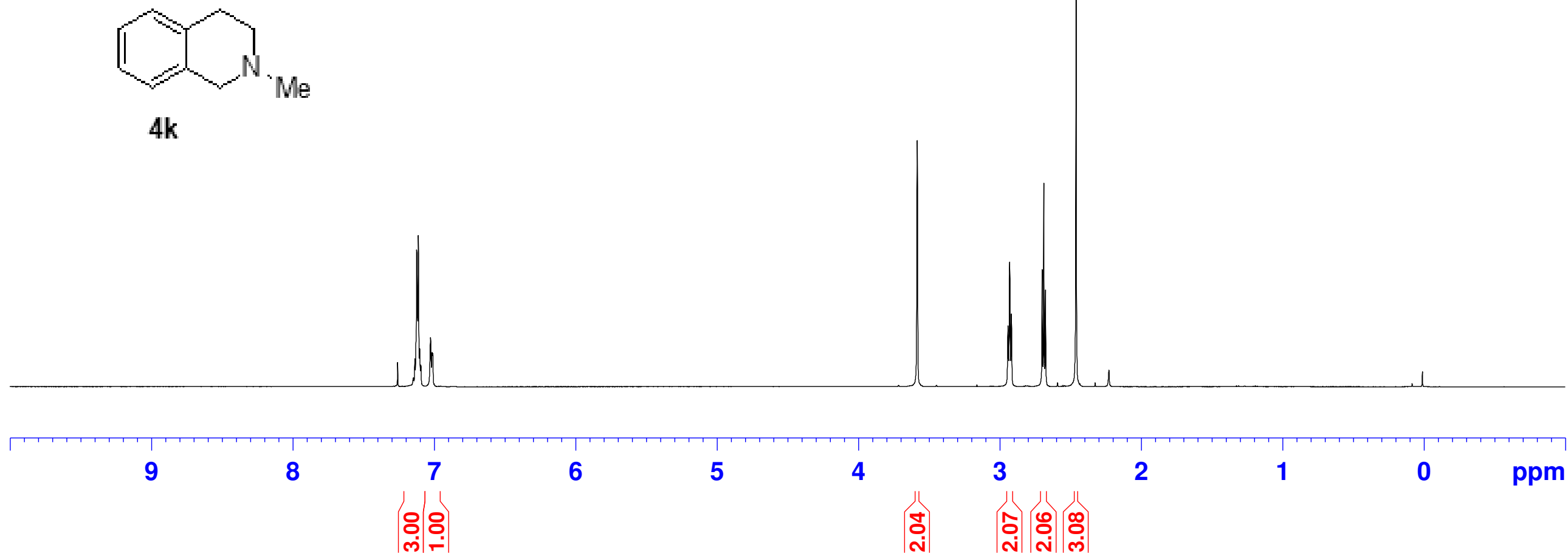
2-methyl-1, 2, 3,4-tetrahydroisoquinoline C13CPD CDCl3
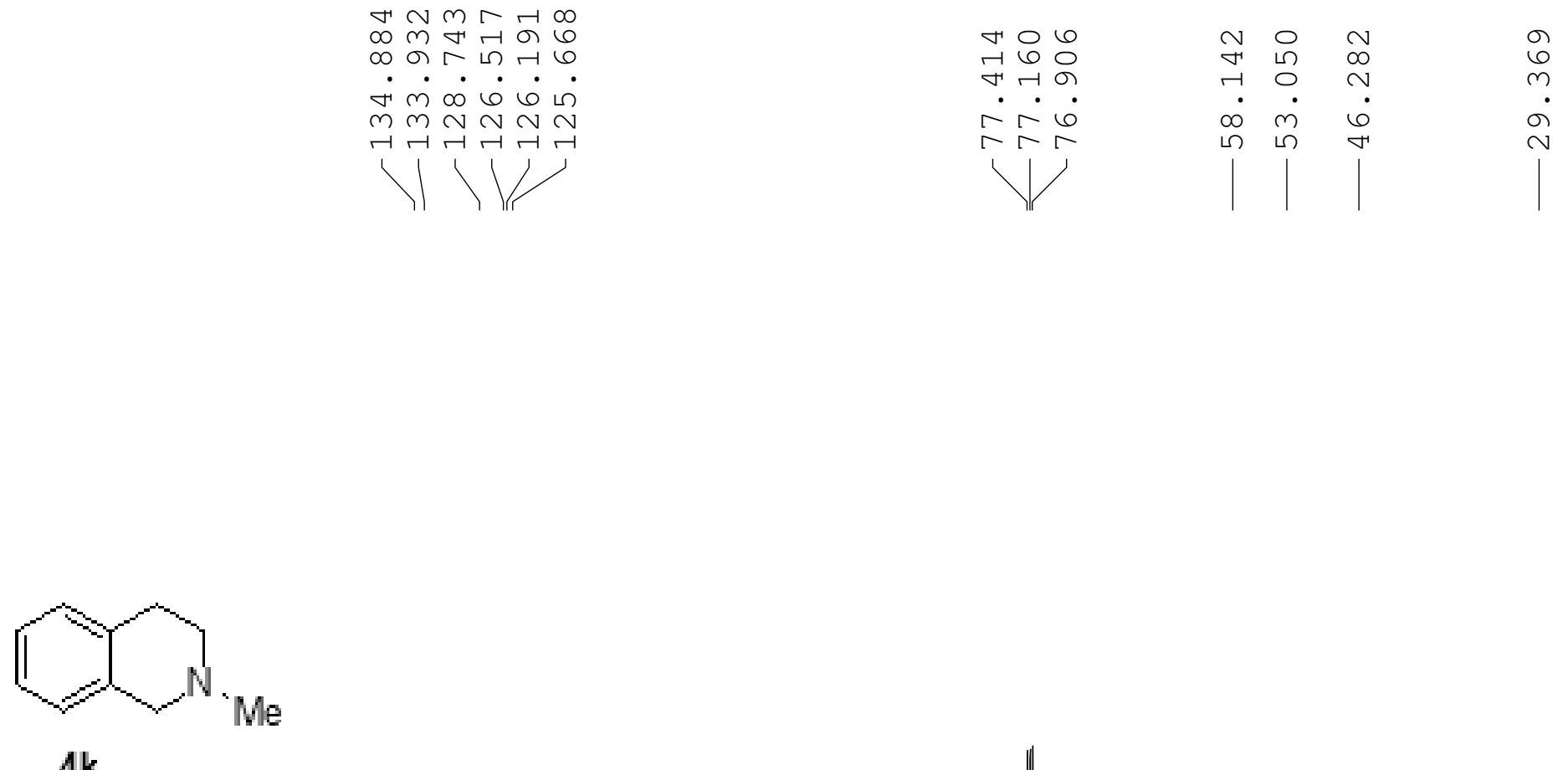

$4 k$

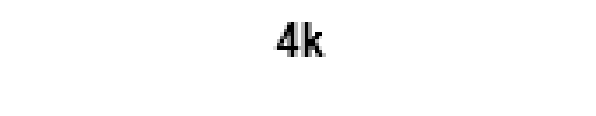

r1

110

10090

80

$80 \quad 70 \quad 60$

$\begin{array}{llllllll}50 & 40 & 30 & 20 & 10 & 0 & \text { ppm }\end{array}$


1-methyl-4-(3-(1-methylpiperidin-4-yl)propyl)piperidine Proton $\mathrm{CDCl} 3$

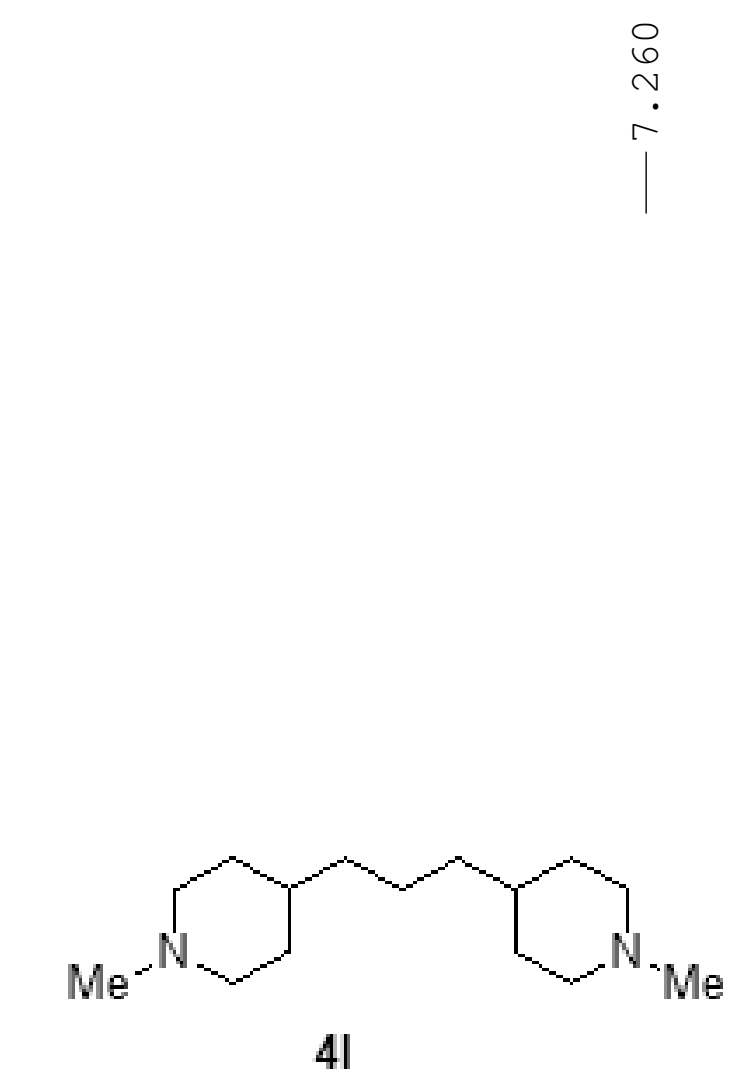

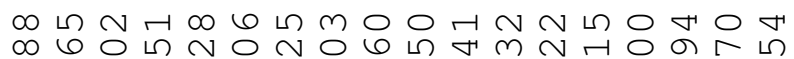
$n \in$
0
$\infty$ र $>13$

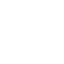

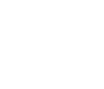


1-methyl-4-(3-(1-methylpiperidin-4-yl)propyl)piperidine C13CPD $\mathrm{CDCl} 3$
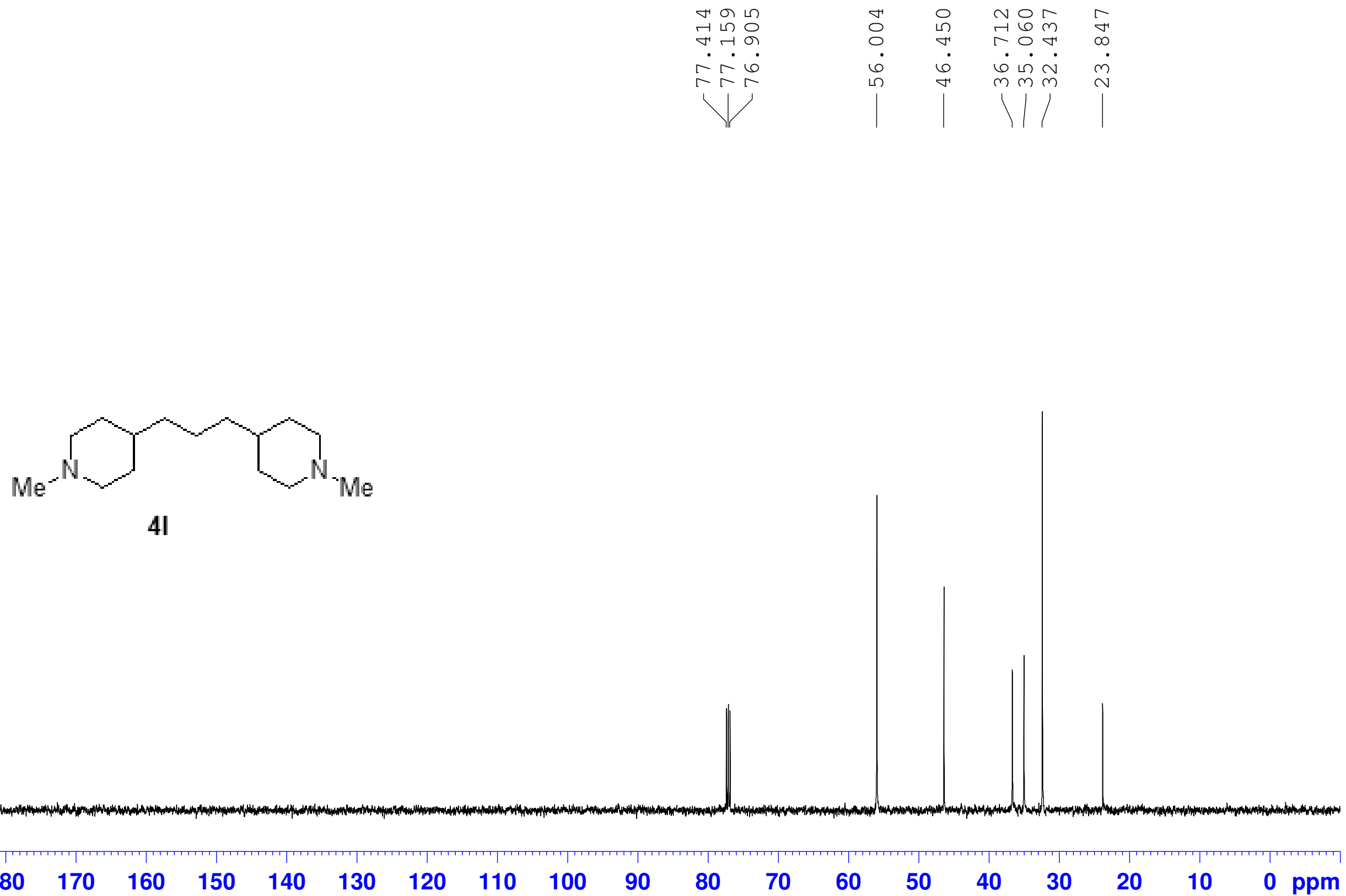

$\begin{array}{lllllllllllllllllllllll}180 & 170 & 160 & 150 & 140 & 130 & 120 & 110 & 100 & 90 & 80 & 70 & 60 & 50 & 40 & 30 & 20 & 10 & 0 & \text { ppm }\end{array}$


Nomotan

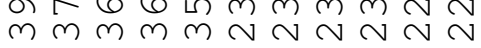

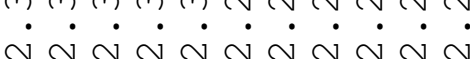

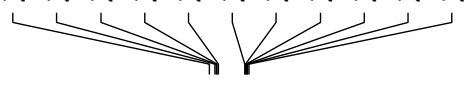<smiles>CN1CCN(C)CC1</smiles>

$4 \mathrm{~m}$ 
1,4-dimethylpiperazine

C13CPD CDCl3
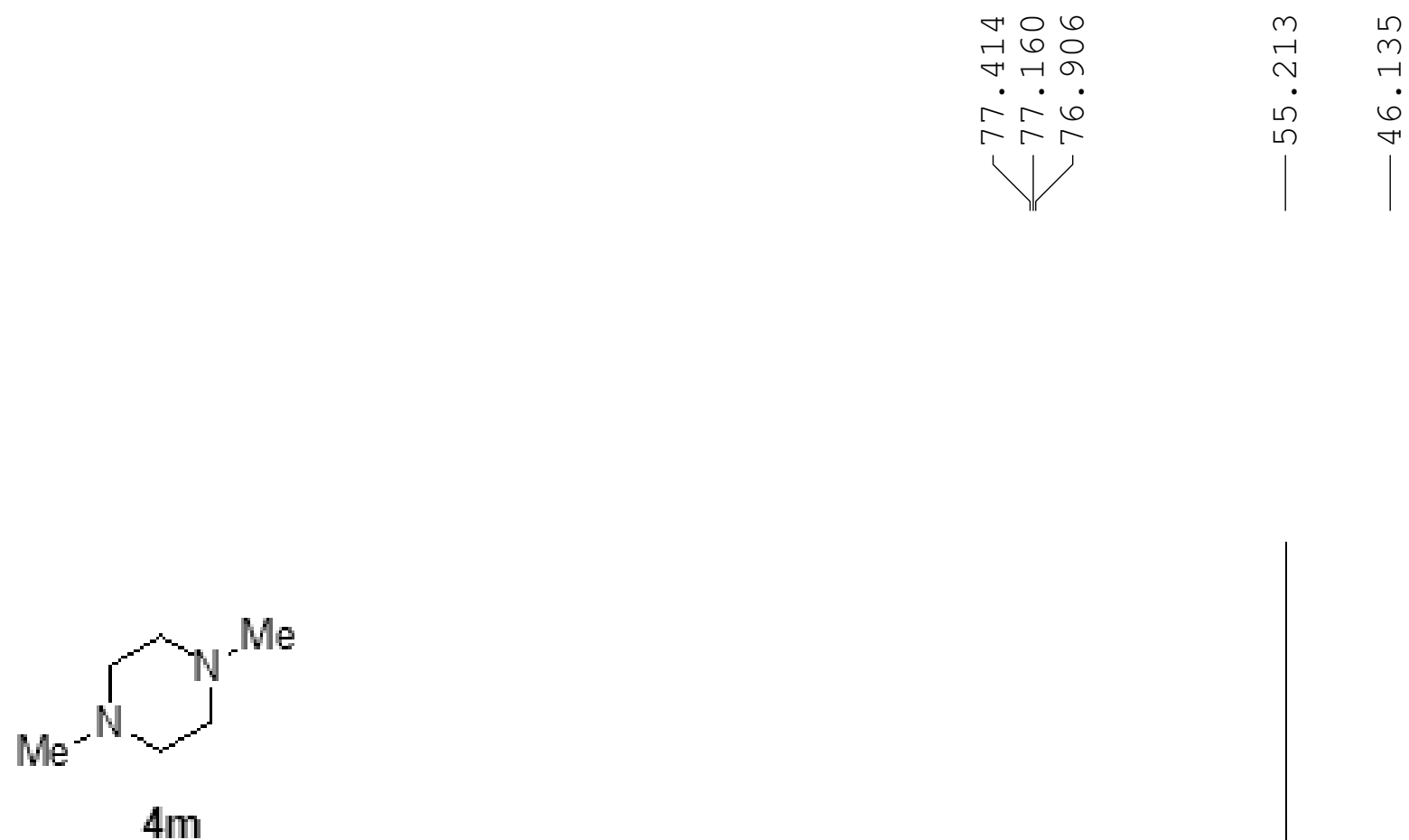

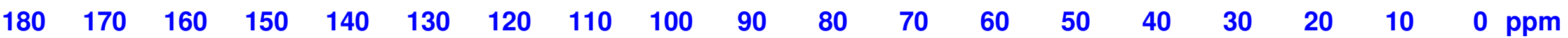


$\circ \% \circ \infty m \infty m \infty m$

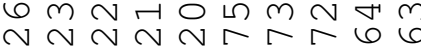

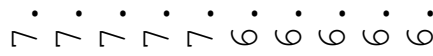

3

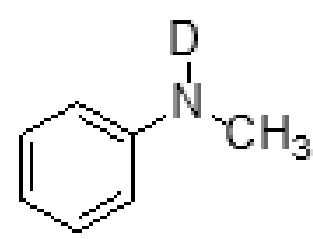

5
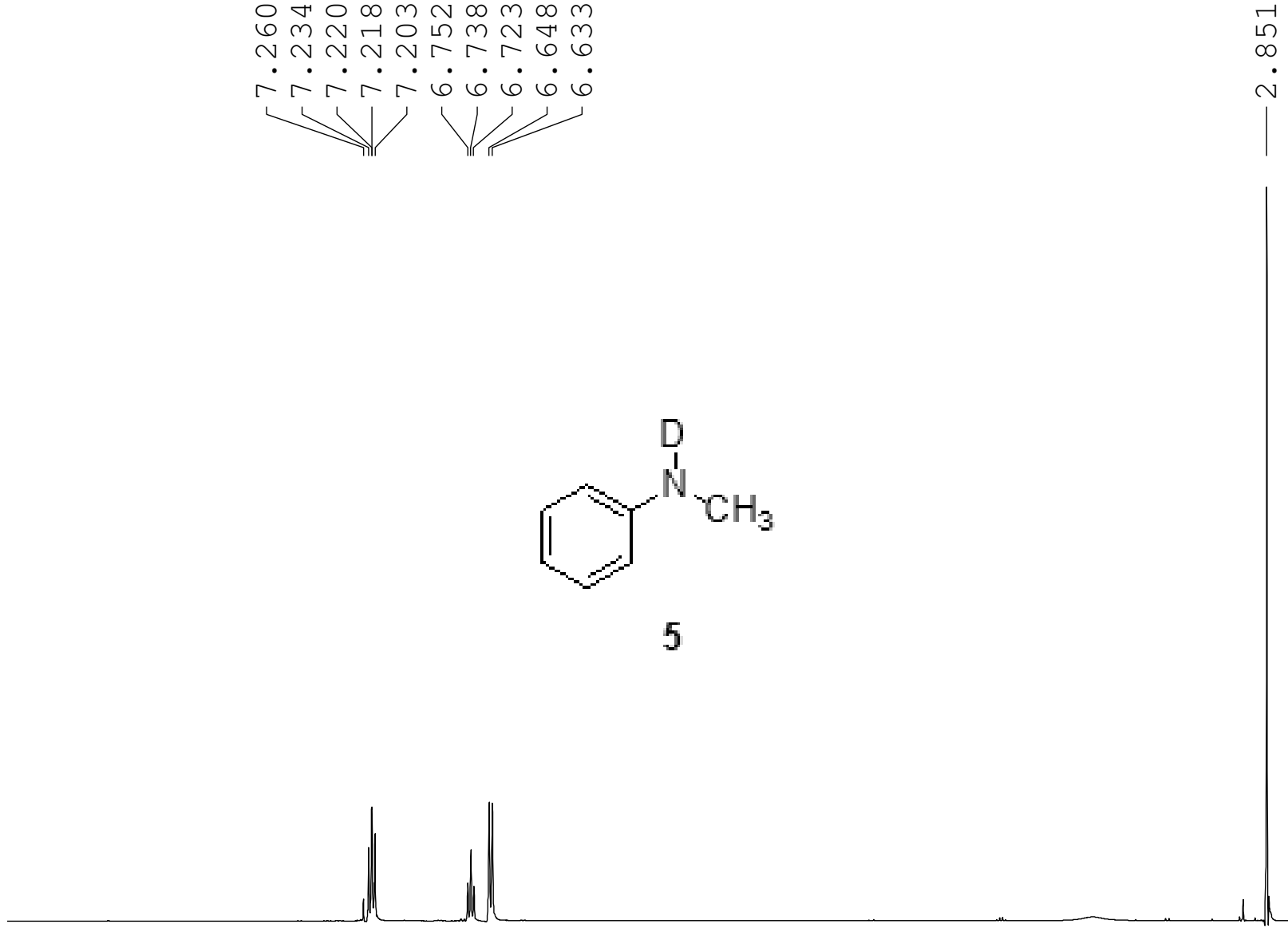

$\begin{array}{lllllllll}8.5 & 8.0 & 7.5 & 7.0 & 6.5 & 6.0 & 5.5 & 5.0 & 4.5\end{array}$

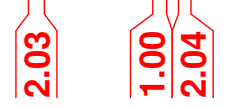

0 


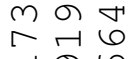

निक्ष

$\therefore \dot{0}$

11
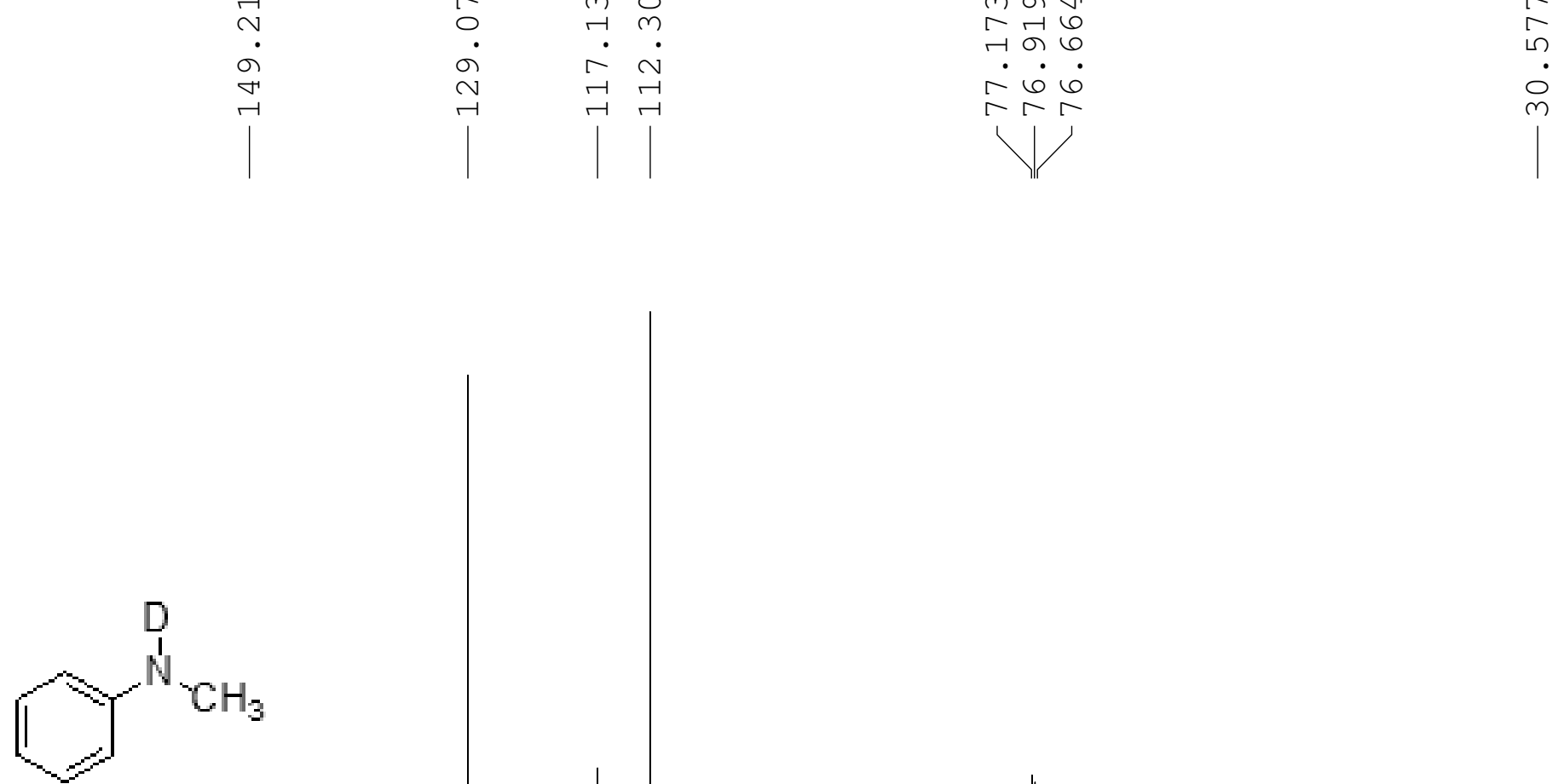

5

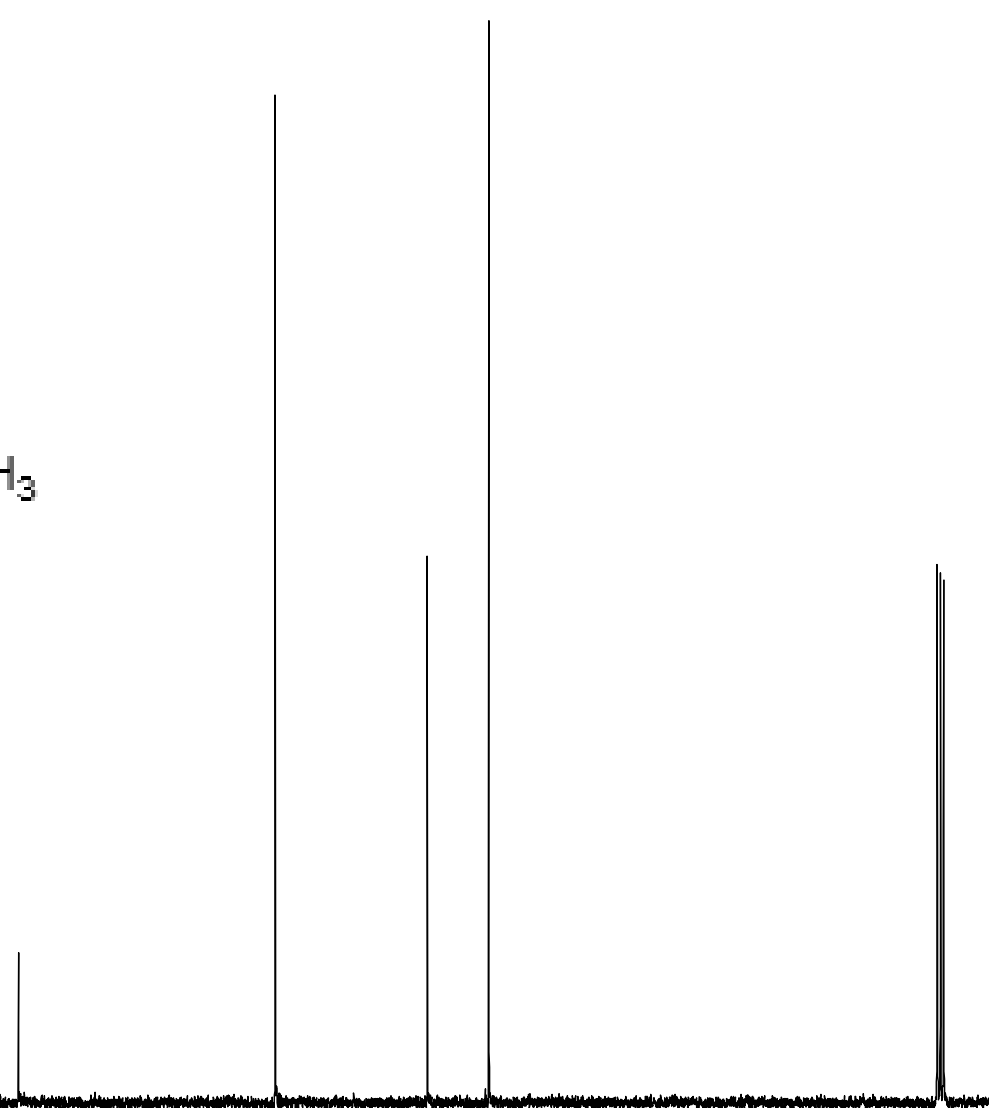

180

160

150

$140 \quad 130$

120

110100

90

80

70

60

50

40 


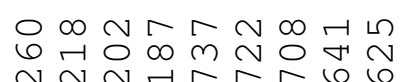

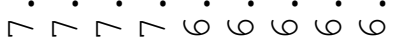

4

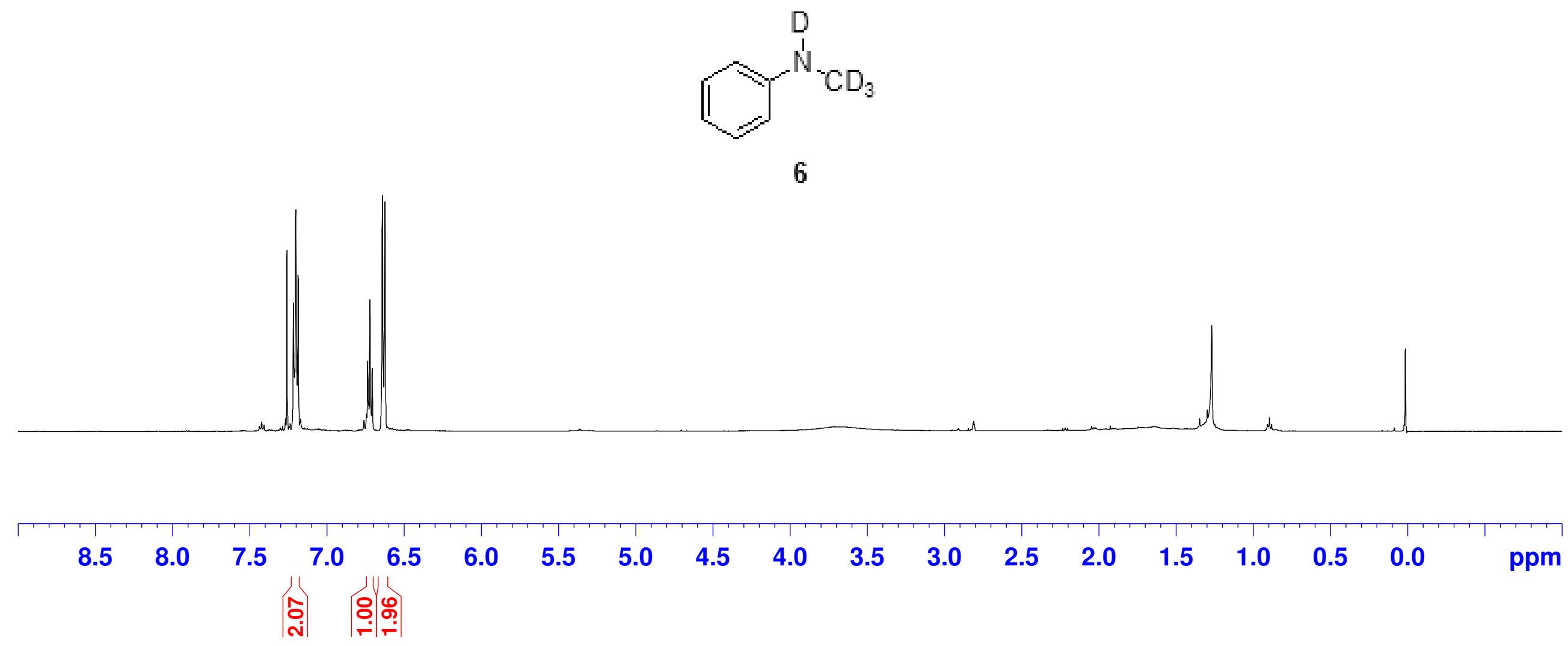


<smiles>[2H]N(c1ccccc1)c1ccccc1</smiles>

6

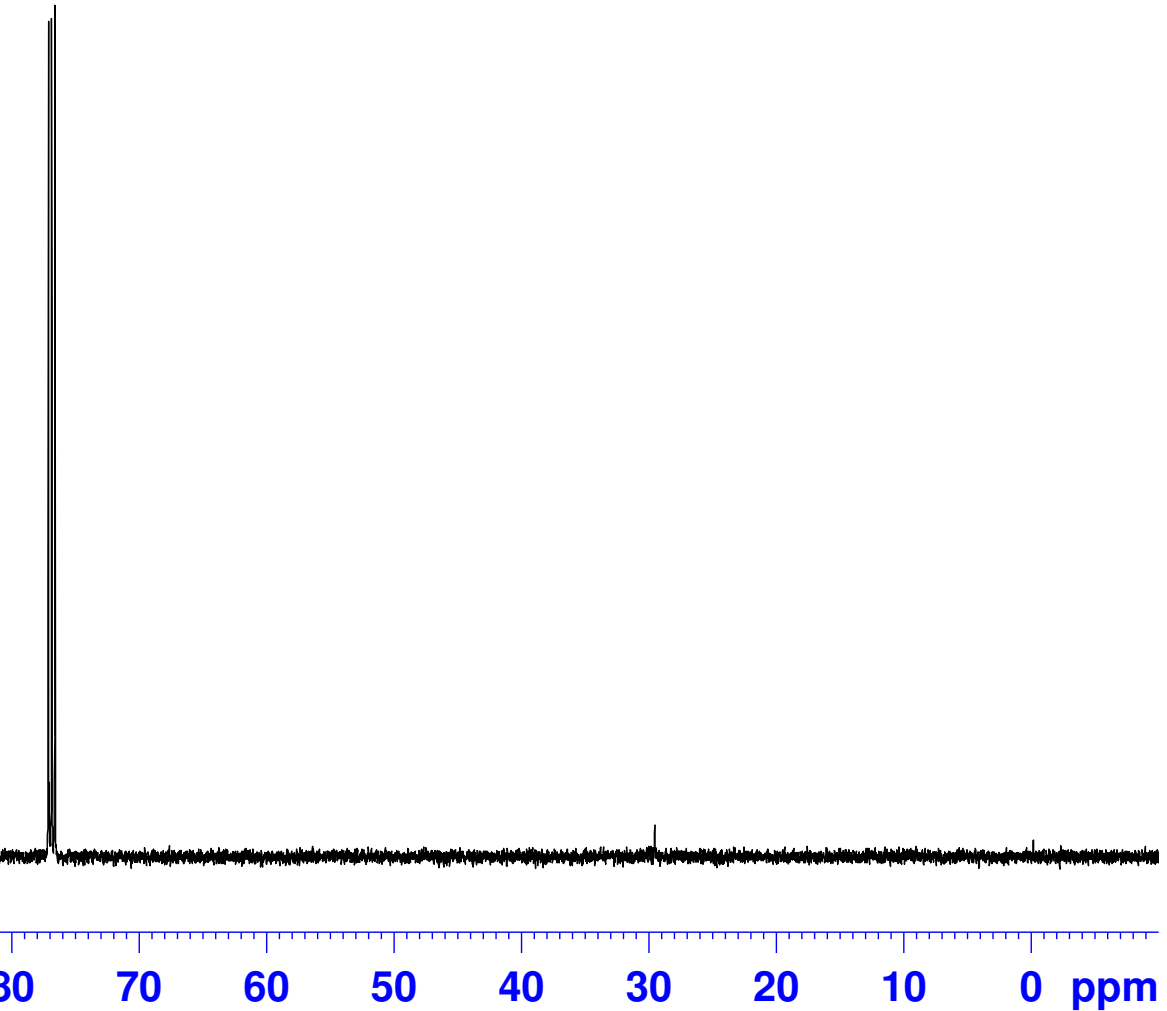




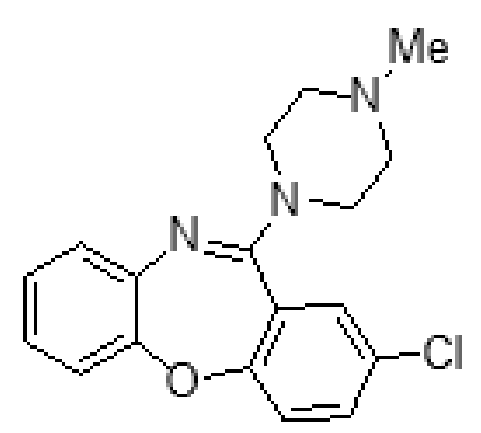

8

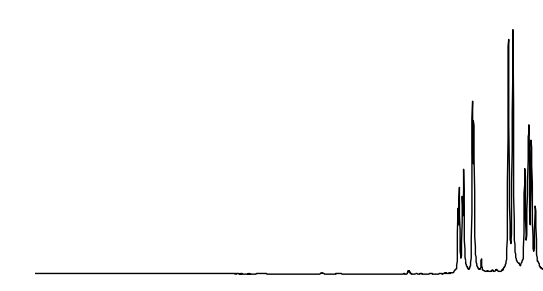

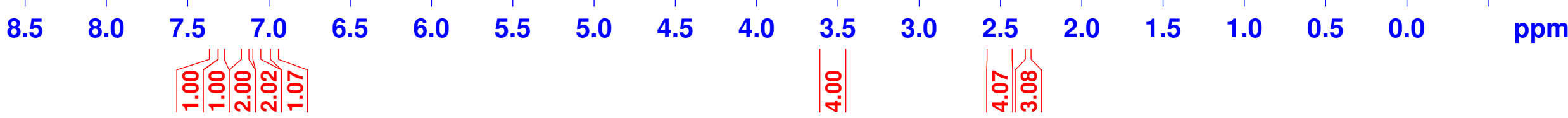


2-Chloro-11-(4-methyl-1-piperazinyl) dibenz [b,f] [1,4] oxazepine C13CPD CDCl3
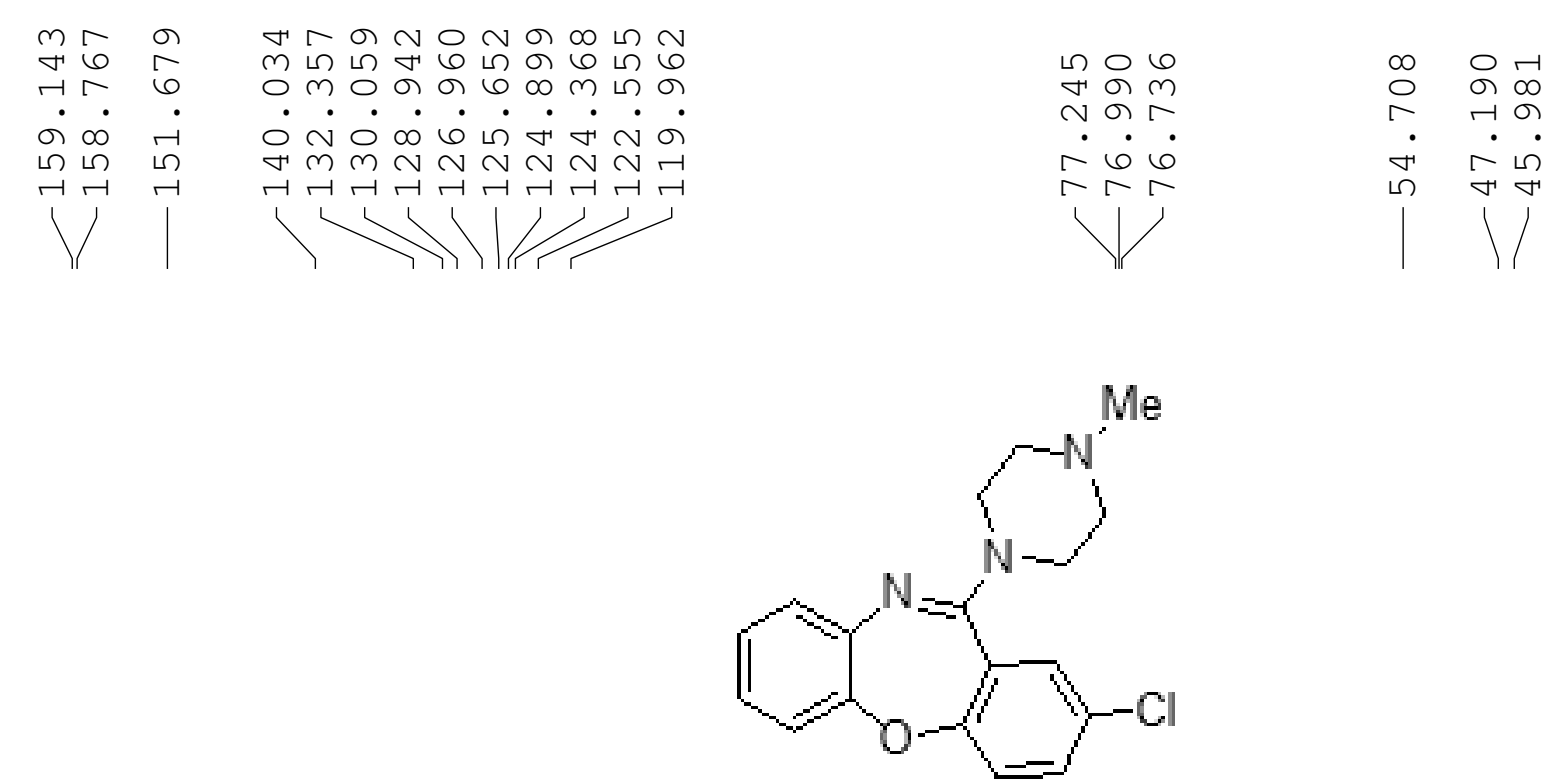

8

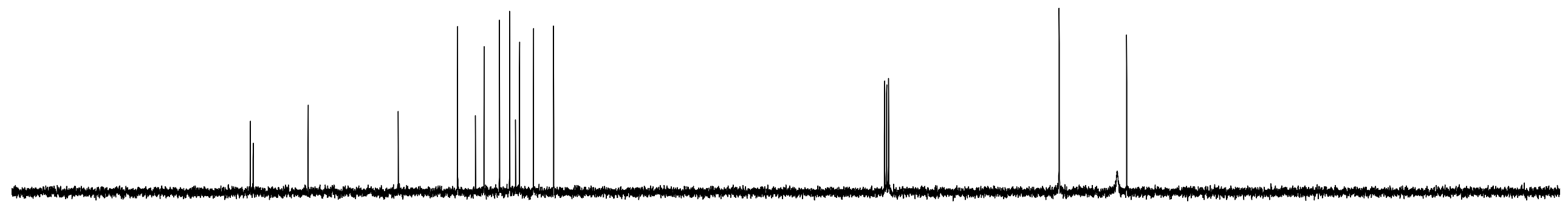

180

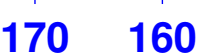

150

140130

120

110

100

90

80

$70 \quad 60$

50

40

30

20

10

0 ppm 


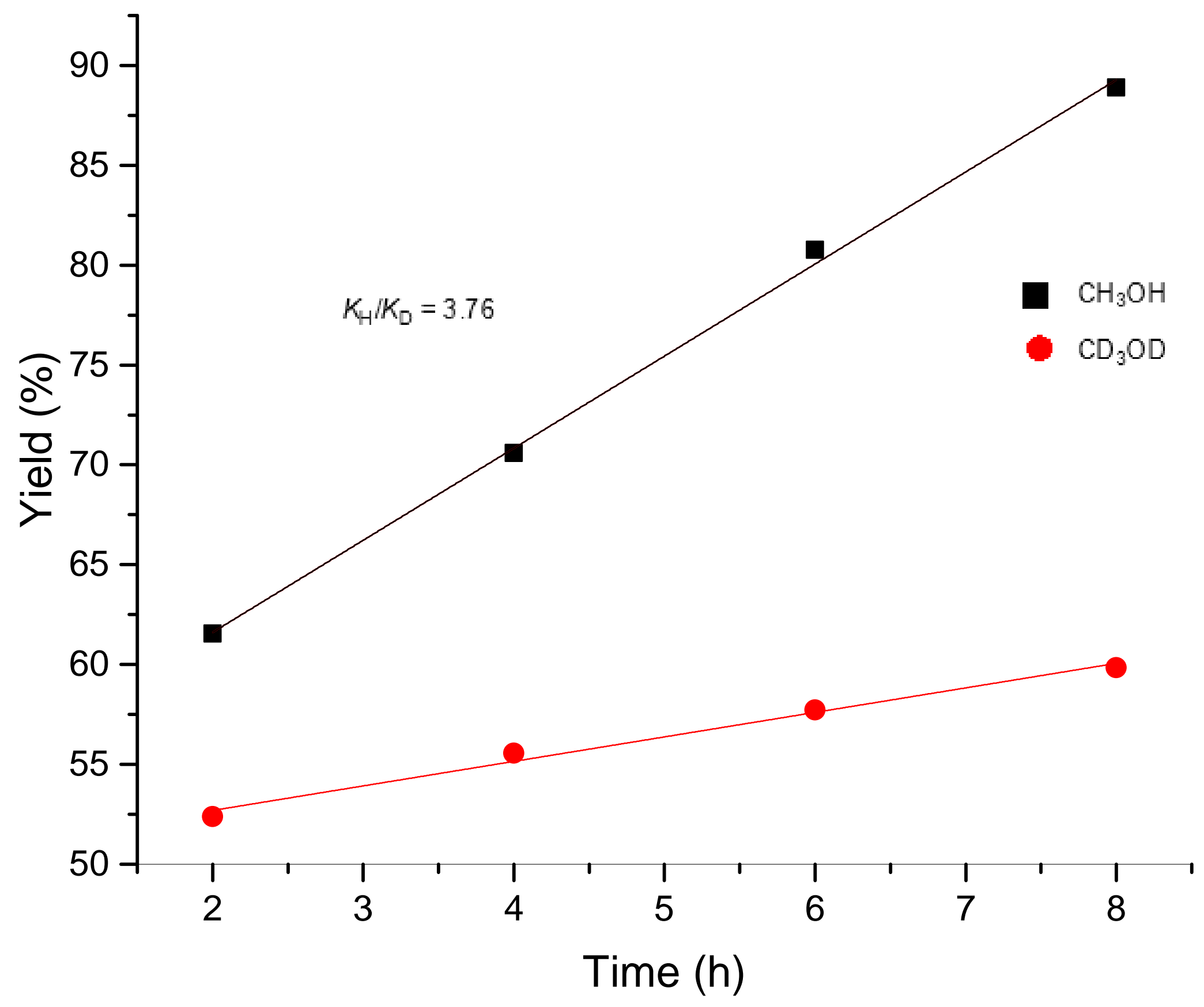




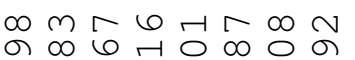

H4Hत

$\therefore \therefore \dot{\sim} \dot{\theta} \dot{\theta} \dot{0}$

$M$

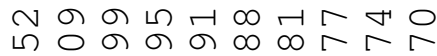

g markrkrta

$\dot{m} \dot{\sim} \dot{\sim} \dot{\sim} \dot{\sim} \dot{\sim} \dot{\sim} \dot{\sim} \dot{\sim}$

$1+$
のள $\infty r r r r r$

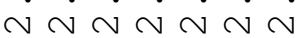

$M M 1 /$

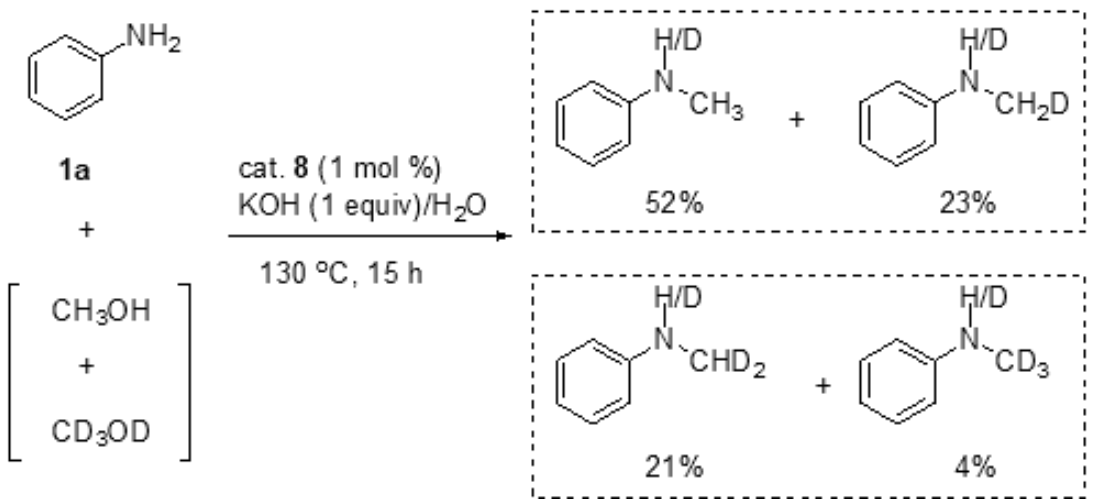

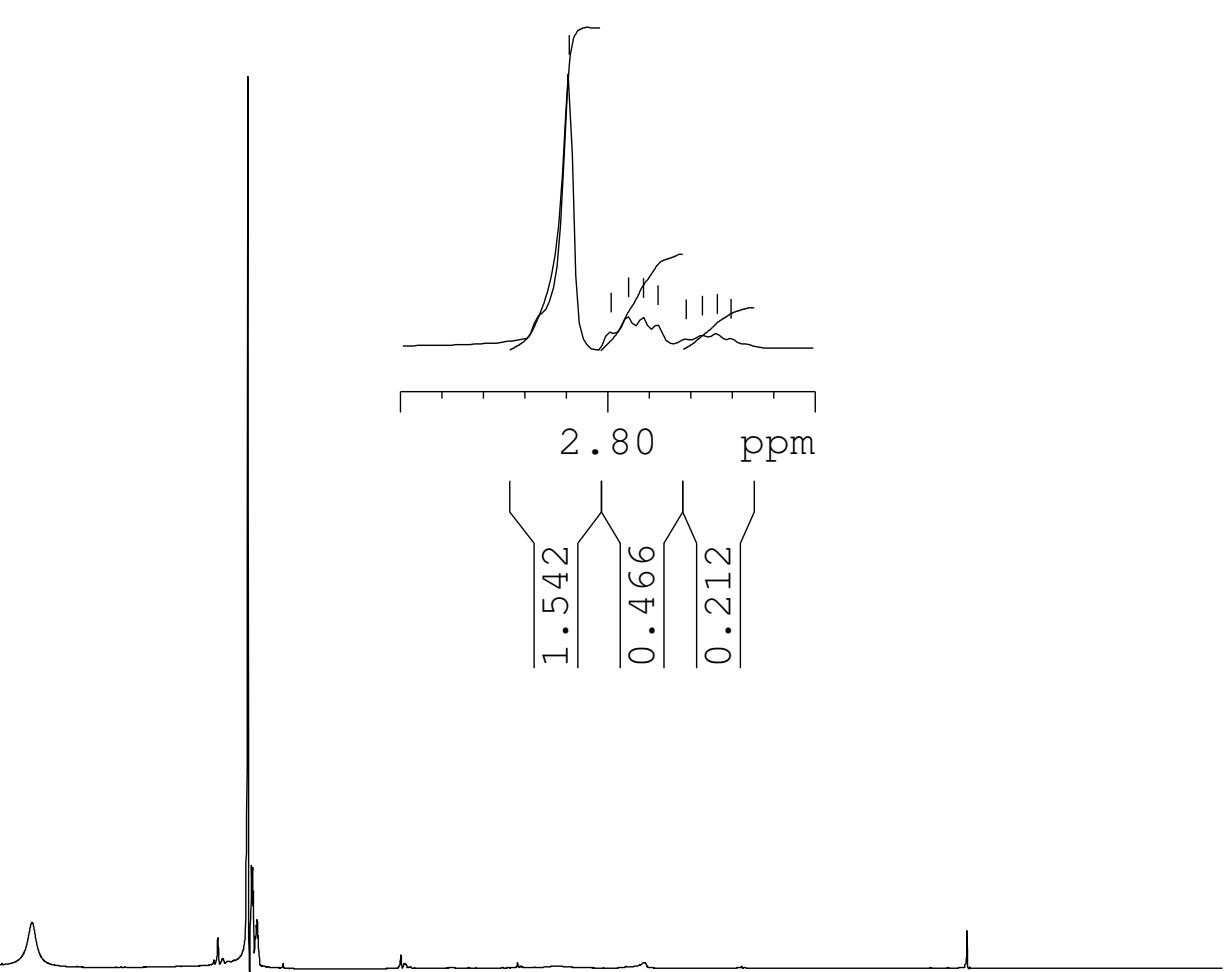
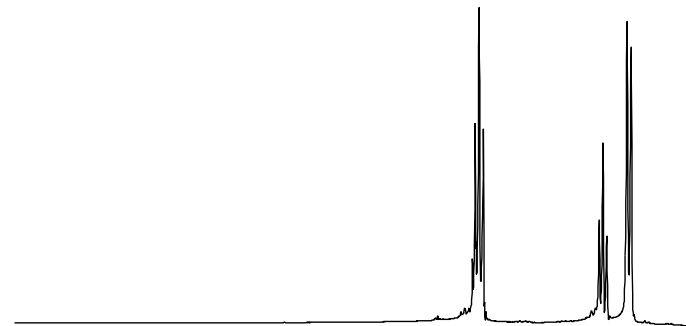

\begin{tabular}{|c|c|c|c|c|c|c|c|c|c|c|c|c|c|c|c|c|c|}
\hline 8.5 & 8.0 & 7.5 & 7.0 & 6.5 & 6.0 & 5.5 & 5.0 & 4.5 & 4.0 & 3.5 & 3.0 & 2.5 & 2.0 & 1.5 & 1.0 & 0.5 & 0.0 \\
\hline & & & בְ. & $\left|\begin{array}{c}0 \\
\hdashline \\
\hdashline\end{array}\right|$ & & & & & & & & & & & & & \\
\hline
\end{tabular}

